\title{
ADAPTIVE DECISION FEEDBACK EQUALIZATION FOR MULTI-Gbps DATA LINKS
}

by

\author{
Alaa R. Abdullah \\ Bacelor of Science, University of Technology, Baghdad, Iraq, 1989 \\ Master of Science, Ryerson University, Toronto, Canada, 2010
}

\author{
A dissertation \\ presented to Ryerson University \\ in partial fulfillment of the \\ requirement for the degree of \\ Doctor of Philosophy \\ in the Program of \\ Electrical and Computer Engineering
}

Toronto, Ontario, Canada, 2014

(C)Alaa R. Abdullah, 2014 


\section{Author's Declaration For Electronic Submission of A Dissertation}

I hereby declare that I am the sole author of this dissertation. This is a true copy of the dissertation, including any required final revisions, as accepted by my examiners. I authorize Ryerson University to lend this dissertation to other institutions or individuals for the purpose of scholarly research.

I further authorize Ryerson University to reproduce this dissertation by photocopying or by

other means, in total or in part, at the request of other institutions or individuals for the purpose of scholarly research.

I understand that my dissertation may be made electronically available to the public. 


\title{
Abstract
}

\section{Adaptive Decision Feedback Equalization For Multi-Gbps Data Links}

\author{
(C)Alaa R. Abdullah, 2014 \\ Doctor of Philosophy \\ Electrical and Computer Engineering \\ Ryerson University
}

Channel equalization combats the effects of the imperfection of wire channels. This dissertation deals with adaptive decision feedback channel equalization. The dissertation starts with an in depth study of the challenges encountered in the design of adaptive DFE and techniques that address these challenges. Various 2-dimensional eye-opening monitors (EOMs) based adaptive DFE are proposed and implemented. A novel 2-dimensional hexagon EOM is proposed and its effectiveness is validated using simulation. A simplified and power efficient 2-dimensional hexagon EOM is also introduced. Both EOMs are capable of differentiating the severity of the violation of the minimum eye-opening so as to allow the DFE to take different actions adaptively and achieve desired eye-opening more rapidly. A maximum-jitter EOM-based adaptive DFE is also introduced to greatly reduce system complexity. The adaptive DFE is taped out in a 130nm $1.2 \mathrm{~V}$ CMOS technology and finally an improved adaptive engine that outperforms DFE utilizing sign-sign least-mean-square is proposed. 


\section{Acknowledgements}

I am heartily thankful to my supervisor, Professor Fei Yuan, for his wonderful guidance throughout all the stages of my $\mathrm{PhD}$ studies. Without his continued encouragement and support, this dissertation would not have been possible. I am also thankful for the excellent example Professor Fei Yuan has provided as a successful researcher, wonderful teacher and role model.

I wish to express my sincere gratitude to my co-supervisor Dr. Andy Ye who helped and supported me in every aspect during the completion of my studies.

I offer my regards and blessings to my colleagues and staff at Integrated Circuits and Systems Research Group at Ryerson University who provided me with valuable technical supports during my studies.

Last but not least, I would like to gratefully acknowledge the financial support of Ryerson University especially Electrical and Computer Engineering department and Ontario Graduate Scholarship OGS for their financial support during my studies. 


\section{Dedication}

To my wonderful wife, daughter and sons, Nissreen, Nursan, Hussin, Yousif and Saif, for their unconditional love, enormous support and continuous encouragement... 


\section{Contents}

1 Introduction 1

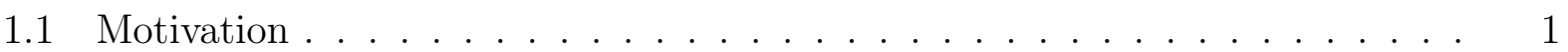

1.2 Objective of The Dissertation . . . . . . . . . . . . . . . . 4

1.3 Chapter Organization . . . . . . . . . . . . . . . . 5

1.4 Inter-Symbol Interference . . . . . . . . . . . . . . . . . . . . . 5

1.5 Channel Equalization . . . . . . . . . . . . . . . . 6

1.5.1 Near-End Channel Equalization . . . . . . . . . . . . . . 7

1.5.2 Far-End Channel Equalization . . . . . . . . . . . . . . . 10

1.6 Decision Feedback Equalization . . . . . . . . . . . . . . . . . 13

1.7 Chapter Summary . . . . . . . . . . . . . . . . . 17

1.8 Contributions of The Dissertation . . . . . . . . . . . . . . . . . 17

1.9 Organization of The Dissertation . . . . . . . . . . . . . 18

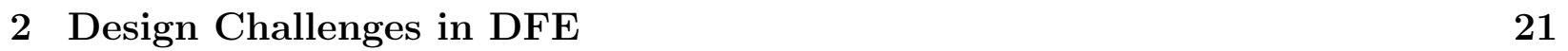

2.1 Bit Error Rate Test . . . . . . . . . . . . . . . . . . . . . . . . . . 21

2.2 Timing Constraints . . . . . . . . . . . . . . . . . 22

2.3 Timing Alignment . . . . . . . . . . . . . . . . . . . . . . . . 24

2.4 Error Propagation . . . . . . . . . . . . . . . 26

2.5 Calibration of Slicer . . . . . . . . . . . . . . . . . . . . . . . 27

2.6 Delay Cells . . . . . . . . . . . . . . . . . . 30

2.7 Arithmetic Operation . . . . . . . . . . . . . . . . . . 31

2.8 Channels with Severe Dispersity . . . . . . . . . . . . . . 33 
2.9 Power Consumption . . . . . . . . . . . . . . . . . . . . . . . 34

2.10 Adaptive Decision Feedback Equalization . . . . . . . . . . . . . . . 34

2.10 .1 Least-Mean-Square Adaptive DFE . . . . . . . . . . . . . . . . 35

2.10 .2 Eye-Opening Adaptive DFE . . . . . . . . . . . . . . . . 37

2.10 .3 Jitter-Based Adaptive DFE . . . . . . . . . . . . . . . . 38

2.10 .4 Comparison . . . . . . . . . . . . . . . . . . 40

2.11 Chapter Summary . . . . . . . . . . . . . . . . . . . 40

3 2-Dimensional Hexagon Eye-Opening Monitor 41

3.1 Eye-Opening Monitors . . . . . . . . . . . . . . . . . . . . . . . . . 41

3.1 .1 One-Dimensional EOMs . . . . . . . . . . . . . . . . . . . . 42

3.1 .2 Two-Dimensional EOMs . . . . . . . . . . . . . . . . 43

$3.1 .3 \quad$ Jitter-Based EOMs . . . . . . . . . . . . . . . . . . . . . 43

3.1 .4 Eye-Pattern Monitors . . . . . . . . . . . . . . . . . 45

3.2 Proposed Two-Dimensional EOM . . . . . . . . . . . . . . . 46

3.2.1 Drawbacks of Rectangular EOM . . . . . . . . . . . . . . . 46

3.2 .2 Diamond EOM . . . . . . . . . . . . . . . . . 47

3.2 .3 Hexagon EOM . . . . . . . . . . . . . . . . . . . 47

3.2 .4 Discussion . . . . . . . . . . . . . . . . . . . . . 50

3.3 Implementation . . . . . . . . . . . . . . . . . . 53

3.4 Chapter Summary . . . . . . . . . . . . . . . . . . . . . . 60

4 2-Dimensional Half-Hexagon Eye- Opening Monitor 61

4.1 Half Hexagon Eye-Opening Monitor . . . . . . . . . . . . . . . . . . . . . 61

4.2 Implementation . . . . . . . . . . . . . . . . . . 63

4.3 Simulation Results . . . . . . . . . . . . . . . . . . . 66

4.4 Chapter Summary . . . . . . . . . . . . . . . . . . . . 71

5 Adaptive DFE Uing 2-Dimensional Hexagon EOM 73

5.1 The Principle . . . . . . . . . . . . . . . . . . . . . . . . 74 
5.2 The Algorithm . . . . . . . . . . . . . . . . . . . . 75

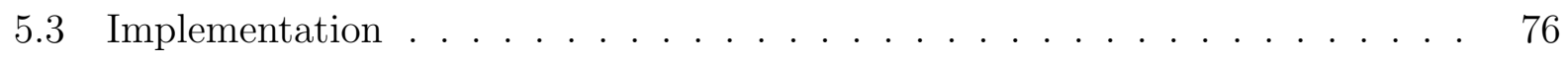

5.3 .1 The Architecture . . . . . . . . . . . . . . . . 76

$5.3 .2 \quad$ Slicer. . . . . . . . . . . . . . . . . . . . . . . . . . . . 78

5.3 .3 Error Detection Unit . . . . . . . . . . . . . . . . . . 78

5.3 .4 Digital-To-Analog Converter . . . . . . . . . . . . . . . . 81

$5.3 .5 \quad$ XOR Gate . . . . . . . . . . . . . . . . . . . . . . 82

5.4 Simulation Results . . . . . . . . . . . . . . . . . . . . . 83

5.5 Chapter Summary . . . . . . . . . . . . . . . . . . . 86

6 Maximum-Jitter Adaptive DFE $\quad 91$

6.1 Maximum-Jitter Adaptive DFE . . . . . . . . . . . . . . . . . . . . . . 92

6.1 .1 The Principle . . . . . . . . . . . . . . . . . . . . . . . . . . 92

6.2 Implementation . . . . . . . . . . . . . . . . . . . 94

6.2 .1 Error Detection Unit . . . . . . . . . . . . . . . . . . . . . 94

6.2 .2 Digital-to-Analog Converter . . . . . . . . . . . . . . 95

6.3 Simulation Results . . . . . . . . . . . . . . . . . . . . 96

6.4 Drawbacks of Adaptive DFE Using SS-LMS or EOM . . . . . . . . . . 99

6.4.1 Drawbacks of Adaptive DFE Using SS-LMS . . . . . . . . . . . . 99

6.4 .2 Drawbacks of Adaptive DFE Using EOM . . . . . . . . . . . . . 101

6.5 The Solution . . . . . . . . . . . . . . . . . . . . . 102

6.6 Simulation Results . . . . . . . . . . . . . . . . . . . . . . . . . 104

6.7 Chapter Summary . . . . . . . . . . . . . . . . . . . . . 107

7 Conclusions and Future Work 113

7.1 Conclusions . . . . . . . . . . . . . . . . . . . . . . . 113

7.2 Future Work . . . . . . . . . . . . . . . . . . . . . . . . . 114

7.3 List of Publications . . . . . . . . . . . . . . . . . . 115 


\section{List of Figures}

1.1 Dependence of data rate on the minimum channel length of MOS transistors.

1.2 (a) Frequency response of a 30" trace on a Nelco4000-13SI board [1]. (b) Frequency response of a 16" Tyco legancy backplane with two daughter cards [4]. $(\mathrm{c}, \mathrm{d})$ Frequency response and impulse response of a highly reflective backplane [5] (Copyright CIEEE). . . . . . . . . . . . . . . . .

1.3 Pre-cursors, main cursor, and post-cursors of data symbol at the far end of a wire channel. . . . . . . . . . . . . . . . . . . . . . 6

1.4 Inter-symbol interference in multi symbols. . . . . . . . . . . . . . . . . . 6

1.5 Near-end channel equalization with first-order pre-emphasis. The added preemphasis tap shortens the duration of the received symbol thereby improving data rate. . . . . . . . . . . . . . . . . . 8

1.6 Frequency response of an equalized channel with pre-emphasis. The highfrequency components of data symbols are boosted prior to their transmission. 9

1.7 Frequency response of an equalized channel using de-emphasis. The lowfrequency components of data symbols are attenuated prior to their transmis-

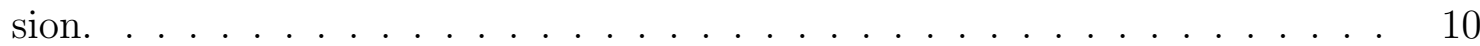

1.8 Continuous-time linear equalizers with inductor series peaking, source degeneration, and negative capacitors. . . . . . . . . . . . . . . 
1.9 Basic configuration and operation of decision feedback equalization. Legends: UI - Delay cell with one unit delay, $v_{s}=v_{i n}-v_{f}$ is the symbol before the slicer. $v_{r e f}$ is the threshold of the slicer, $D_{j=1}$ is the delayed version of $D_{j}$, $c_{1}, \ldots, c_{N}$ are the weighting factors of feedback taps, $T_{s}$ is the symbol time, $H_{j, k}$ denote the $k$ th post-cursor of symbol- $j$ and $H_{0, j}$ the main cursor of symbol- $j$.

2.1 Dependence of horizontal eye-opening on BER. . . . . . . . . . . . . . 22

2.2 Tap-1 feedback is implemented using loop-unrolling. . . . . . . . . . . . . 23

2.3 Half-rate decision feedback equalization. Tap-1 is implemented using loopunrolling while the remaining taps are implemented using direct feedback. Note that complementary clocks are used for upper and lower paths of DFE.

2.4 Post-cursor cancellation error due to timing error of delay stages. . . . . . . 25

2.5 Error in data slicing affects the removal of post-cursors. . . . . . . . . . . . 27

2.6 Clocked re-generative sense amplifier as slicer [37] . . . . . . . . . . . 28

2.7 (a) Clocked re-generative sense amplifier as delay cell [25], (b) Clocked currentmode delay cells [44]. . . . . . . . . . . . . . . . . . 30

2.8 Current-mode summer with resistive load. . . . . . . . . . . . . . . . 32

2.9 Least-mean-square adaptive DFE. . . . . . . . . . . . . . . . 35

2.10 Configuration of SS-LMS adaptive DFE. . . . . . . . . . . . . 36

2.11 Eye-opening monitors. . . . . . . . . . . . . . . . . . . 38

2.12 1-dimensional Eye-opening adaptive DFE [53] . . . . . . . . . . . . . 38

2.13 Relation between vertical opening and edge jitter. . . . . . . . . . . . 39

3.1 One dimensional EOM quantified by $V_{H}$ and $V_{L}$ at $t_{d}$ vertically. Two-dimensional EOM quantified by $V_{H}$ and $V_{L}$ vertically and $t_{1}$ and $t_{2}$ horizontally. . . . . 42

3.2 Relation between vertical opening and edge jitter. . . . . . . . . . . . 43

3.3 Rectangular and diamond EOMs. . . . . . . . . . . . . . . 46

3.4 Hexagon EOM. . . . . . . . . . . . . . . . . . . . 48 
3.5 (a) Hexagon EOM that has the same vertical opening but different horizontal opening as that of rectangular EOM. (b) Hexagon EOM that has the same horizontal opening as that of rectangular EOM. . . . . . . . . . . . . . . 49

3.6 Asymmetrical hexagon EOMs. . . . . . . . . . . . . . . . . 51

3.7 Serial link with a hexagon EOM. For the purpose of comparison, a rectangular EOM in parallel with the hexagon EOM is also employed. . . . . . . . . . . 53

3.8 Simulated output of the error generators of rectangular EOM with $v_{\text {in,max }}>$ $V_{H}$ and $v_{i n, m i n}<V_{L} \ldots \ldots \ldots \ldots \ldots$

3.9 Simulated output of the error generators of rectangular EOM with $v_{\text {in,max }}<$ $V_{H}$ and $v_{i n, m i n}<V_{L} \ldots \ldots \ldots \ldots$. . . . . . . . . . . . . . . . . . . . . .

3.10 Simulated output of the error generators of rectangular EOM with $v_{\text {in,max }}>$ $V_{H}$ and $v_{i n, m i n}>V_{L} \ldots \ldots \ldots \ldots$

3.11 Simulated output of the error generators of hexagon EOM with $v_{i n, \min }<V_{L}$ and $v_{i n, \max }>V_{H} \ldots \ldots \ldots \ldots$

3.12 Simulated output of the error generators of hexagon EOM with $v_{i n, \max }<V_{H}$

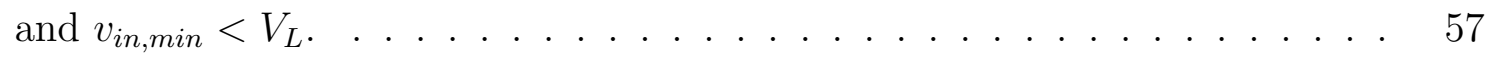

3.13 Simulated output of the error generators of hexagon EOM with $v_{i n, \min }>V_{L}$ and $v_{i n, \max }>V_{H} \ldots \ldots \ldots \ldots \ldots \ldots$

3.14 Simulated output of the error generators of rectangular EOM and hexagon EOM with a 1-mm channel, $v_{i n, \min }<V_{L}$, and $v_{i n, \max }>V_{H} \ldots \ldots$. . . . 58

3.15 Simulated output of the error generators of rectangular EOM and hexagon EOM with a $40-m m$ channel. . . . . . . . . . . . . . . . . . 59

3.16 Output of error generator of hexagon EOM. . . . . . . . . . . . . . 60

4.1 Detection of violation of the minimum eye-opening using half hexagon mask.

4.2 Left : Full and half rectangular Eye-Opening patterns. Right : Full and half hexagon Eye-Opening patterns . . . . . . . . . . . . . . . . 62 
4.3 Serial link with half and full hexagon EOMs. Circuit parameters : $W_{1,2}=$ $6.5 \mu \mathrm{m}, W_{3,4}=4 \mu \mathrm{m}, W_{5}=50 \mu \mathrm{m} . L=0.13 \mu \mathrm{m}$ for all transistors. $V_{b}=0.7 \mathrm{~V}$ with tail current $I_{s s}=2 \mathrm{~mA} \ldots \ldots \ldots \ldots \ldots$

4.4 Clocked comparator. Circuit parameters : Circuit parameters : $W_{1,2}=4.5 \mu \mathrm{m}$, $W_{3,5}=4 \mu \mathrm{m}, W_{4,6,7,8}=8 \mu \mathrm{m}, W_{9,10}=1 \mu \mathrm{m}, W_{11,12}=1 \mu \mathrm{m}, W_{13,14}=2 \mu \mathrm{m}$, and $W_{15,16,17}=60 \mu \mathrm{m} . L=0.13 \mu \mathrm{m}$ for all transistors. $I_{s s}=2 \mu \mathrm{A}, V_{b}=0.8 \mathrm{~V} \ldots .64$

4.5 Effect of duty-cycle distortion on hexagon EOM. . . . . . . . . . . . 65

4.6 Schematic of XOR2. Circuit parameters: $W_{1,2}=3 \mu m, W_{7,8}=1.5 \mu m$, and $W_{9}=50 \mu m . L=0.13 \mu m$ for all transistors, $V_{b}=0.8 V \ldots \ldots \ldots$

4.7 Serial link with half and full rectangular EOMs. The same circuit and channel parameters as those in Fig. 4.3 are used. . . . . . . . . . . . . . . . .

4.8 Simulated output of full hexagon EOM with $v_{i n, \min }<V_{L}$ and $v_{i n, \max }>V_{H}$. No error is detected at $S t_{2}$ and $S t_{3}$. Top plot : Received data. 2nd-5th plots : Sampling clocks $S t_{2} \sim S t_{4}$. 6th-9th plots : Error outputs $e 1 h \sim e 4 h . \ldots$

4.9 Simulated output of half hexagon EOM with $v_{i n, \min }<V_{L}$ and $v_{i n, \max }>V_{H}$. No error is detected at $S t_{2}$ and $S t_{3}$. Top plot : Received data. 2nd-3rd plots : Sampling clocks $S t_{2} \sim S t_{2}$. 4th-5th plots : Error outputs $e 1 h \sim e 2 h . \ldots$.

4.10 Simulated output of full hexagon EOM with $v_{i n, \max }<V_{H}$ and $v_{i n, \min }<V_{L}$. Errors are detected at $S t_{2}$ and $S t_{3}$. Top plot: Received data symbol. 2nd-5th plots : Sampling clocks $S t_{2} \sim S t_{4}$. 6th-9th plots: Error outputs $e 1 h \sim e 4 h$. .

4.11 Simulated output of half hexagon EOM with $v_{i n, \max }<V_{H}$ and $v_{i n, \min }<V_{L}$. Errors are detected at $S t_{2}$. Top plot : Received data symbol. 2nd-3rd plots : Sampling clocks $S t_{2} \sim S t_{3} .4$ th-5th plots : Error outputs $e 1 h \sim e 2 h . \ldots$

4.12 Simulated output of half hexagon EOM with a 200-mm channel. Top plot: Received data. 2nd and 3rd plots : Sampling clocks $S t_{2} \sim S t_{3}$. 4th and 5th plots : Output of half-hexagon EOM. . . . . . . . . . . . . . 
4.13 Simulated output of half rectangular EOM with a 200-mm channel. Top plot : Received data. 2nd plot : Sampling clock $S t_{1}$. 3rd plot : Output of halfrectangular EOM. . . . . . . . . . . . . . . . . . . . . 70

4.14 Simulated output of hexagon EOM with a 200-mm channel. Top plot : sampling clock. 2nd plot : Received data symbol and the minimum vertical eye-opening. 3rd plot : Output of EOM at process corners (TT: typical nMOS/typical pMOS; FF: fast nMOS/fast pMOS; FS: fast nMOS/slow pMOS, SF: slow nMOS/fast pMOS, and SS: slow nMOS/slow pMOS). Bottom plot : Output of EOM with temperture from $-20 \sim 80 \mathrm{C} \ldots \ldots \ldots . . . .70$

5.1 (a) Violation of the minimum eye-opening at $t_{1}$ only. (b)Violation of the minimum eye-opening at $t_{1}$ and $t_{2}$. (c) No violation of the minimum hexagon EOM but violation of the minimum rectangular EOM exists. . . . . . . . . 74

5.2 Search engine of proposed hexagon EOM based adaptive DFE. . . . . . . . 76

5.3 DFE core. Transistor sizes: $W_{1,2}=4 \mu \mathrm{m}, W_{3,4,5,6}=3 \mu \mathrm{m}, W_{7,8}=1 \mu \mathrm{m}, W_{9}$ $=40 \mu \mathrm{m}$, and $W_{10,11}=30 \mu \mathrm{m}, L=0.13 \mu \mathrm{m}$. For all transistors biasing: $I_{s s}=$

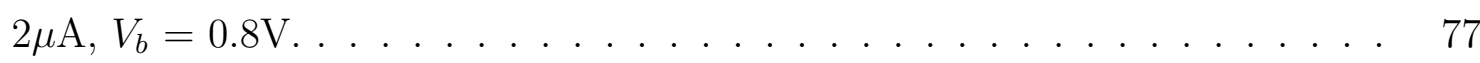

5.4 Error detection units. . . . . . . . . . . . . . . . . . . . 79

5.5 Severity of violation with hexagon EOM. . . . . . . . . . . 80

5.6 Configuration (top) and schematic (bottom) of variable step-size DAC. Charge pump $M_{3}-M_{8}$ is enabled when $e_{t 1}=1$. Charge pump $M_{9}-M_{14}$ is enabled when

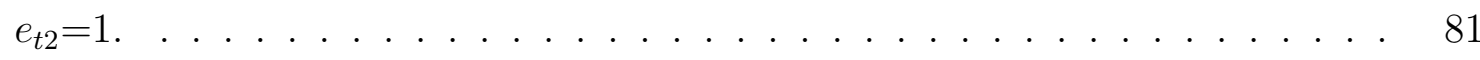

5.7 Proposed adaptive DFE. . . . . . . . . . . . . . . . . . . 83

5.8 Response of channels of length $35 \mathrm{~mm}$ (left) and $55 \mathrm{~mm}$ (right) to a pulse input at the near end of the channel. . . . . . . . . . . . . 84

5.9 Tap coefficients of adaptive DFE with hexagon and rectangular EOMs. . . . 85

5.10 Error detection of rectangular and hexagon adaptive DFE. . . . . . . . 86 
5.11 Waveform of data before (left) and after (right) the proposed adaptive DFE. Top: channel length=100 mm. Middle: channel length=500mm. Bottom: channel length=1M. . . . . . . . . . . . . . . . . . 87

5.12 Error detection and tap coefficients of proposed adaptive DFE with $35 \mathrm{~mm}$ channel length. . . . . . . . . . . . . . . . . . . . . . 88

5.13 Error detection and tap coefficients of proposed adaptive DFE with $55 \mathrm{~mm}$ channel length. . . . . . . . . . . . . . . . . . . . 88

6.1 Jitter-based of error detection. (a,c) - No error exists. (b,d) - Errors exist. . 92

6.2 Adaptive engine of proposed jitter-based adaptive DFE. . . . . . . . . . . . 94

6.3 Error Detection Unit. Channel parameters : Microstrip with width $10 \mu \mathrm{m}$, height $2 \mu \mathrm{m}$, and length $80 \mu \mathrm{m}$. Dielectric constant of field oxide : $\epsilon_{r}=5 . \quad . \quad 95$

6.4 Schematic of digital-to-analog converter (DAC) . . . . . . . . . . 96

6.5 Adaptation process of proposed jitter-based adaptive DFE with a $20 \mathrm{~mm}$ channel. . . . . . . . . . . . . . . . . . . . . . . . . . . 97

6.6 Waveforms of data symbols without (left) and with (right) the proposed 2-tap jitter-based adaptive DFE. Channel lengths : (a) $100 \mathrm{~mm}$, (b) 1M, and (c) 2M. 98

6.7 (a) : Simulated eye diagram of the data link. Channel length : $50 \mathrm{~mm}$. Top : Data conveyed to the channel. Middle : Data at the far-end of the channel without the proposed DFE. Bottom : Data at the far-end of the channel with the proposed DFE. (b) : The left edge of the simulated eye diagram of the data link. Channel length : $50 \mathrm{~mm}$. Top : Data conveyed to the channel. Middle : Data at the far-end of the channel without the proposed DFE. Bottom : Data at the far-end of the channel with the proposed DFE. . . . . . . . . . . . . 99

6.8 LMS tap coefficients. . . . . . . . . . . . . . . . . . . . . . . 100

6.9 LMS step size. . . . . . . . . . . . . . . . . . . . . . . . . . . . . . 101

6.10 One-direction step size of EOM. . . . . . . . . . . . . . . . . . . 102

6.11 Drawback of one-direction step size of EOM. . . . . . . . . . . . . . 102 
6.12 The schematic of proposed adaptive DFE including the proposed adaptive engine. . . . . . . . . . . . . . . . . . . . . 103

6.13 Search engine of proposed new adaptive engine for adaptive DFE. . . . . . . 104

6.14 Serial link including two error detection units. . . . . . . . . . . . . . . 105

6.15 Inverter: schematic (left) and layout (right) . . . . . . . . . . . . . 108

6.16 NOR2: schematic (left) and layout (right) . . . . . . . . . . . . 108

6.17 XOR2: schematic (left) and layout (right) . . . . . . . . . . . . 109

6.18 Delay cell: schematic (left) and layout (right). . . . . . . . . . . . . . . 109

6.19 Clocked-comparator: schematic (left) and layout (right) . . . . . . . . . 110

6.20 Non-clocked-comparator: schematic (left) and layout (right) . . . . . . . . . 110

6.21 Adaptive engine: schematic (left) and layout (right) . . . . . . . . . . . 111

6.22 DFE core: schematic (left) and layout (right) . . . . . . . . . . . . 111

6.23 Layout of maximum-jitter adaptive DFE. . . . . . . . . . . . . . . . . 112 


\section{List of Tables}

1.1 Data rate of serial links utilizing decision feedback equalization. Channel loss is measured at half baud-rate frequency. . . . . . . . . . . . . . . . . 20

3.1 Eye-opening monitors . . . . . . . . . . . . . . . . . . . . . . . 45

4.1 Reference comparison of proposed works . . . . . . . . . . . . . . . 71

4.2 Reference comparison of adaptive DFEs . . . . . . . . . . . . 71

5.1 Performance of proposed hexagon eye-opening monitor adaptive DFE (2 Meter FR4 channel). . . . . . . . . . . . . . . . . . . 89

6.1 Performance of proposed jitter adaptive DFE (1 meter channel length). . . . 107 


\section{Table of Abbreviations and Symbols}

\begin{tabular}{||l|l|l|l||}
\hline \hline Abbr. & Full Name & Symb. & Full Name \\
\hline \hline ADFE & Adaptive DFE & $C_{k}$ & Weighting factor \\
ADC & Analog to digital converter & ck,clk, $\phi$ & Clock signal \\
BER & Bit error rate & $D_{k}$ & Comparator decision \\
CDR & Clock and data recovery & $e_{n}$ & Error signal \\
CTLE & Continuous time linear equalizer & $g_{m}$ & Trans-conductance of CMOS \\
DAC & Digital to analog converter & $H_{n}$ & Equalized feedback cursors \\
DFE & Decision feedback equalizer & $\mathrm{h}$ & Step size \\
EOM & Eye-opening monitor & $t_{n}, S_{t n}$ & Sampling time \\
FFE & Feed forward equalizer & $V_{b}$ & Bias voltage \\
FIR & Finite impulse response & $V_{H}$ & Voltage high \\
Gbps & Gigabit per second & $V_{i n}$ & Input voltage \\
HEOM & Hexagon EOM & $V_{L}$ & Voltage low \\
IIR & Infinite impulse response & $V_{o}$ & Output voltage \\
I.Cs & Integrated circuits & $V_{t h}$ & Threshold voltage \\
ISI & Inter symbol interference & & \\
LFSR & Linear feedback shift register & & \\
LMS & Least mean square & & \\
NRZ & Non return to zero & & \\
PCB & Printed circuit board & & \\
PRBS & Bseudo Random bit stream & & \\
PVT & Process of voltage and temp. variation & & \\
RX & Receiver & & \\
SNR & Signal to noise ratio & \\
SS-LMS & Sign-sign LMS & \\
TX & Transmitter & & \\
UI & Unit interval & & \\
\hline \hline
\end{tabular}




\section{Chapter 1}

\section{Introduction}

This chapter provides a comprehensive review of decision feedback equalization (DFE) for multi-giga-bit-per-second (Gbps) data links. The state-of-the-art of DFE for multi-Gbps serial links reported in the past decade is presented. The imperfections of wire channels, in particular, finite bandwidth, reflection, and cross-talk, and their impact on data transmission are investigated. The fundamentals of both near-end and far-end channel equalization to combat the effect of the imperfection of wire channels at high frequencies are explored. Finally, a detailed examination of the principle, configuration, operation, and limitation of DFE is discussed.

\subsection{Motivation}

The explosive growth of data processed by integrated circuits (ICs) demands that data be transmitted over wire channels (interconnects, vias, connectors, package pins, printed circuit boards PCBs, and coaxial cables) at multiple Gbps. Although increasing the number of wire channels directly improves the total data bandwidth, a large number of parallel channels not only increase the cost of routing, the overall data rate is also affected by clock and data skews caused by the mismatch of the channels [1]. As a result, parallel links are only attractive for short-range data communications such as multi-processor systems, processor-to-memory interfaces, and network switches. Unlike parallel links, serial links transmit data and clock 
using a single wire channel, typically a differential pair to minimize electromagnetic interference with neighboring devices. The elimination of a dedicated channel for clock transmission removes the difficulties associated with clock skew. The use of only a single wire channel also eliminates the bottle neck associated with data skew. Moreover, it greatly reduces the cost associated with routing. As a result, serial links are very attractive in applications such as block-to-block (on-chip), chip-to-chip, chassis-to-chassis, and computer-to-computer links where the distance over which data are transmitted is large and the number of channels available is small.

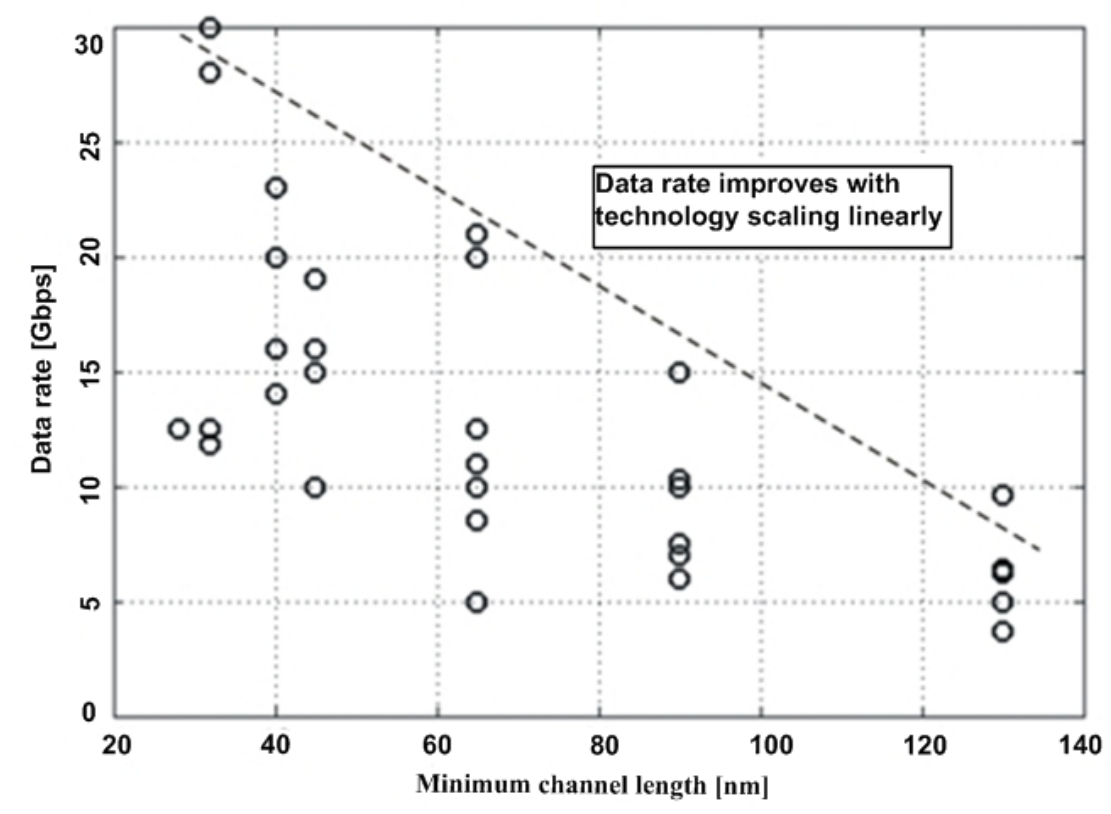

Figure 1.1: Dependence of data rate on the minimum channel length of MOS transistors.

Although the maximum transit frequency of MOS transistors has well exceeded $100 \mathrm{GHz}$, the data rate of serial links is much lower, as evident in Table 1.1, despite the nearly linear improvement of the maximum data rate with technology scaling, as shown in Fig.1.1. The low data rate is mainly due to inter-symbol interference (ISI) arising from channel imperfections with limited bandwidth, reflection, and cross-talk the most critical. 


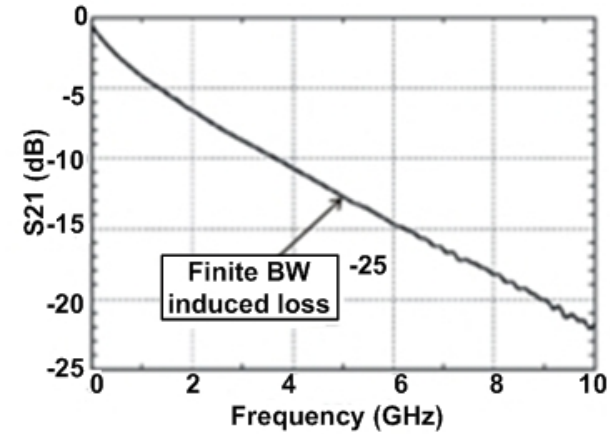

(a)

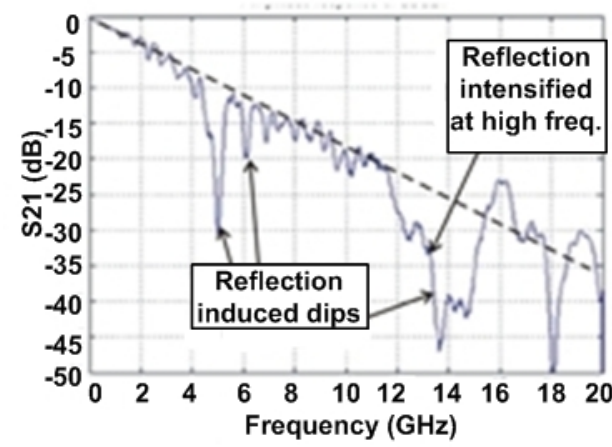

(c)

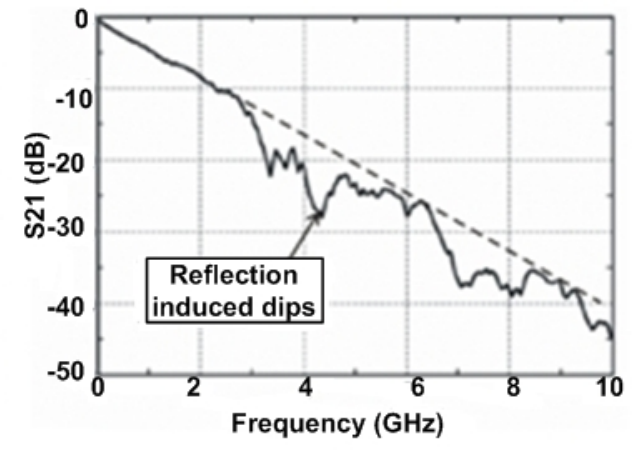

(b)

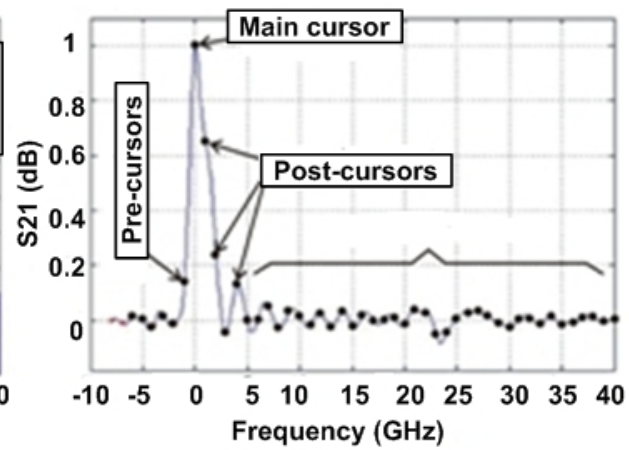

(d)

Figure 1.2: (a) Frequency response of a 30" trace on a Nelco4000-13SI board [1]. (b) Frequency response of a 16" Tyco legancy backplane with two daughter cards [4]. (c,d) Frequency response and impulse response of a highly reflective backplane [5] (Copyright (CIEEE).

The limited bandwidth of channels caused by the rising resistive and dielectric loss of the channels at high frequencies gives rise to a long channel impulse response or equivalently frequency-dependent attenuation, as shown in Fig.1.2(a). Reflection caused by the impedance mismatch of channels, largely due to the inclusion of vias, connectors, and branches in the channels, results in crests and troughs that are non-uniformly distributed over a large number of symbol intervals in channel impulse response or equivalently sharp troughs in frequency domain response, as shown in Fig.1.2(b) [2], [3], [4]. Note that troughs are due to capacitive impedance mismatches. For channels with severe reflection, deep troughs exist, as shown in Fig.1.2(c,d). Crosstalk is primarily due to capacitive and inductive coupling with neighboring devices and manifests itself as crests and troughs in the channel impulse 
response. As a result, received data symbols at the far end of the channel consist of precursors, main cursor, and post-cursors with the number of post-cursors significantly larger than that of the pre-cursors, as shown in Fig.1.2(d). The main cursor is used for data recovery while pre-cursors and post-cursors need to be removed. They can be removed using near-end and far-end channel equalization.

\subsection{Objective of The Dissertation}

The main objective of this dissertation is to develop a new adaptive decision feedback equalizer (DFE) to mitigate the channel imperfections for multi-Gbps serial links. The proposed adaptive DFE consists of three blocks: DFE core, error detection unit (EDU), and adaptive engine (AE). The following list highlights our intended objectives chosen for this dissertation:

1. Modify and improve a summer circuit based on noise, speed, and power consumption to consider it as the core of the proposed adaptive DFE.

2. Create a new technique in the error detection unit (EDU) to efficiently detect the violations of the received data symbols with minimum requirements to safely detect the symbols at the clock and data recovery (CDR) operation.

3. Improve the schematic of EDU to minimize the power consumption.

4. Develop a new adaptive engine (AE) to update the DFE tap coefficients at Giga-bit-per second data rate and improve the convergence time of the adaptation process.

5. Combine the proposed DFE core, error detection unit, and adaptive engine in a comprehensive schematic to form an adaptive DFE and validate it for the speed and power consumption.

6. Prepare a chip layout for the proposed adaptive DFE and fabricate it using an IBM $130 \mathrm{~nm}$ 1.2V CMOS technology. 


\subsection{Chapter Organization}

The remainder of the chapter is organized as the followings: Section 1.4 addresses the effect of inter-symbol interference (ISI) on the integrity of received data signals. Section 1.5 investigates the imperfections of wire channels, in particular, finite bandwidth, reflection, and cross-talk, and their impact on data transmission. The fundamentals of both near-end and far-end channel equalization to combat the effect of the imperfection of wire channels at high frequencies are explored. Section ?? provides a detailed examination of the principle, configuration, operation, and limitation of DFE. Design challenges encountered in the design of DFE for multi-Gbps data links including timing constraints, sampling, error propagation, arithmetic operation, highly dispersive channels, power consumption, and techniques and circuit implementations that address these challenges are studied. Section 2.10 investigates the need for adaptive DFE and the principles of adaptive DFE. The performance of various adaptive DFEs is examined and their pros and cons are compared. The chapter is summarized in Section 2.11. The contributions of the dissertation are provided in Section 1.8 and finally, the organization of the dissertation is given in Section 1.9.

\section{$1.4 \quad$ Inter-Symbol Interference}

Inter-symbol interference (ISI) occurs when data symbols pass through a channel of finite bandwidth. It stretches the rising and falling times exceeding the symbol unit interval (UI) and interferes with previous symbols creating pre-cursors and post-cursor, as shown in Figs.(1.3) and (1.4). At a high data rate, the finite channel bandwidth caused by skin effect, dielectric loss due to the parasitic capacitances between the channel and the substrate, the cross-talk between the channel and its neighbors, and the reflections arising from impedance mismatches at both near and far ends of the channel are the main channel imperfections that contribute most ISI. Due to these effects, the received data symbol at the far end of the channel contains a long tail reducing the opening of the received data eye. 


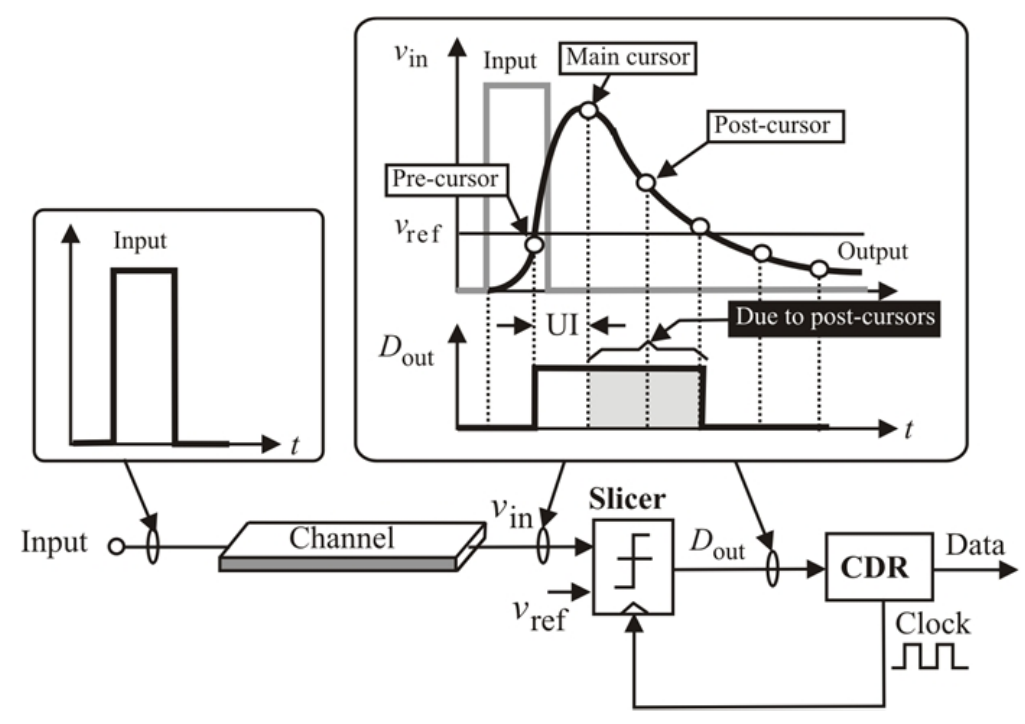

Figure 1.3: Pre-cursors, main cursor, and post-cursors of data symbol at the far end of a wire channel.

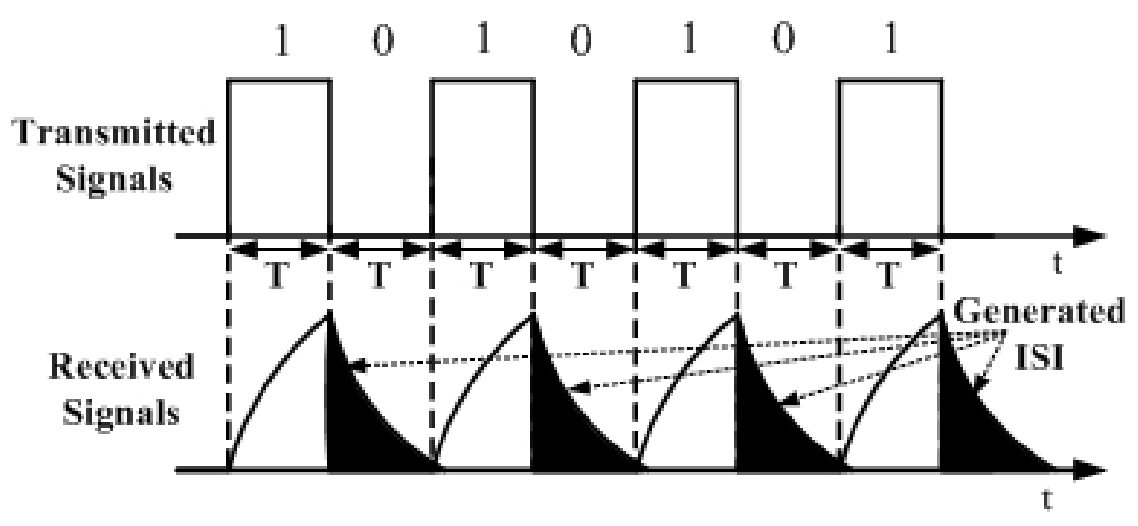

Figure 1.4: Inter-symbol interference in multi symbols.

\subsection{Channel Equalization}

Equalization techniques are widely used to combat ISI. In wire channels, where the channel can roughly be modeled as a low-pass filter, ISI is introduced when the data rate becomes higher than the bandwidth of the channel. This causes the frequency spectrum of the data to 
suffer from unequal magnitude attenuation and phase shifts leading to the distortion of the received signal [6]. Equalization is basically the process of generating a response opposite to the transfer function of the channel. It can be implemented at the channel near end called pre-emphasis, de-emphasis or at the channel far end known as post equalization.

\subsubsection{Near-End Channel Equalization}

Pre-cursors and post-cursors can be removed by boosting the high-frequency components [7], [8] or attenuating the low-frequency components of data symbols [9], [10] prior to transmission. The former increases cross-talk as cross-talk intensifies at high frequencies. The latter reduces the power of the transmitted symbols as the power of non-return-to-zero (NRZ) data is largely concentrated at half baud-rate frequency. Since it increases the relative strength of the high-frequency components of the transmitted signals, cross-talk is also reduced [11]. Near-end channel equalization is often implemented using finite impulse response (FIR) filters that introduce zeros to offset the effect of the poles of the channels [12], [55]. For example, the first-order pre-emphasis FIR filter shown in Fig. 1.2(b) and given by $y(n)=x(n)-a_{1} x(n-1)$ where $x(n)$ and $y(n)$ are the input and output of the FIR filter, respectively, has its transfer function $H_{F I R}(z)=1-a_{1} z^{-1}$. Clearly it introduces a zero at $z=0$ that will impact all the poles of the channels. To demonstrate this, since $\omega T_{s}<<1$ where $T_{s}$ the symbol time, we have $z=e^{s T_{s}} \approx 1+s T_{s}$. As a result,

$$
H_{F I R}(s) \approx\left(1-a_{1}\right)\left(\frac{s}{\omega_{z}}+1\right)
$$

where $\omega_{z}=\left(1-a_{1}\right) /\left(a_{1} T_{s}\right)$. If we model the channel as a first-order low-pass, i.e. $H_{c h}(s)=$ $1 /\left(\frac{s}{\omega_{c h}}+1\right)$ where $\omega_{c h}$ is the channel bandwidth, the transfer function of the equalized channel is given by 


$$
H_{e q}(s)=\left(1-a_{1}\right)\left[\frac{\frac{s}{\omega_{z}}+1}{\frac{s}{\omega_{c h}}+1}\right] .
$$

It becomes apparent that if we choose $\omega_{z}=\omega_{c h}$, the pole of the channel will be canceled by the zero of the pre-emphasis FIR, resulting in a desirable all-pass. Also observed is that since $\left(1-a_{1}\right)<1$, there is a loss of signal energy in pre-emphasis.

Since the characteristics of the channel are not known prior to data transmission, the optimal tap coefficients of pre-emphasis FIR filters can only be obtained if a back channel exists. This constraint undermines the robustness of pre-emphasis channel equalization. Another limitation of pre-emphasis channel equalization is its inability to remove ISI caused by reflection and crosstalk as these ISI manifest themselves as crests and troughs rather than uniformly sloped attenuation, as shown earlier in Fig.1.5. ISI caused by reflection and crosstalk is typically significant when data rate is high and channels contain multiple vias, connectors, and branches (highly reflective channels).
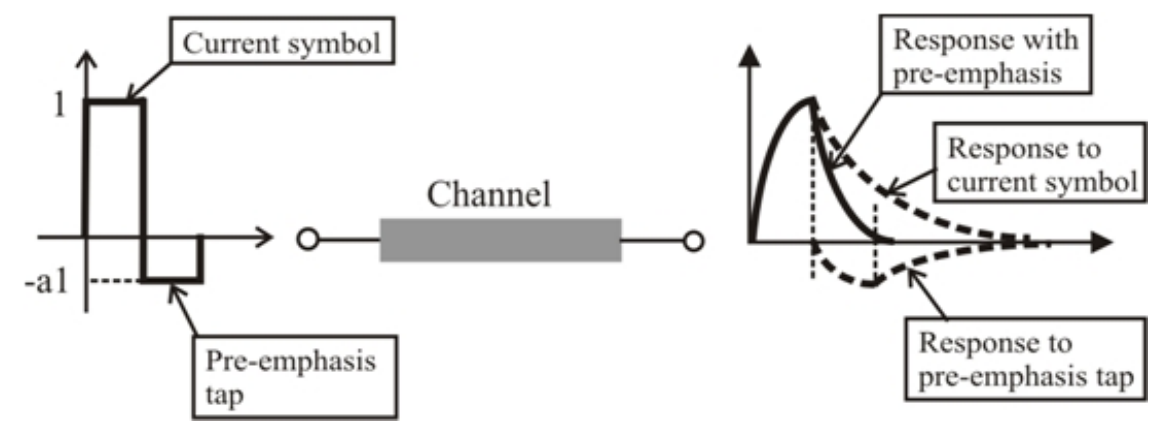

Figure 1.5: Near-end channel equalization with first-order pre-emphasis. The added pre-emphasis tap shortens the duration of the received symbol thereby improving data rate. 


\section{Pre-Emphasis Equalization}

Figure1.6 shows graphically the principle of the pre-emphasis equalization at the channel near end. The high-frequency components of the transmitted symbol are boosted prior to transmission. The result is flattened frequency response of the received data symbol at the channel far end and improved the serial links bandwidth.

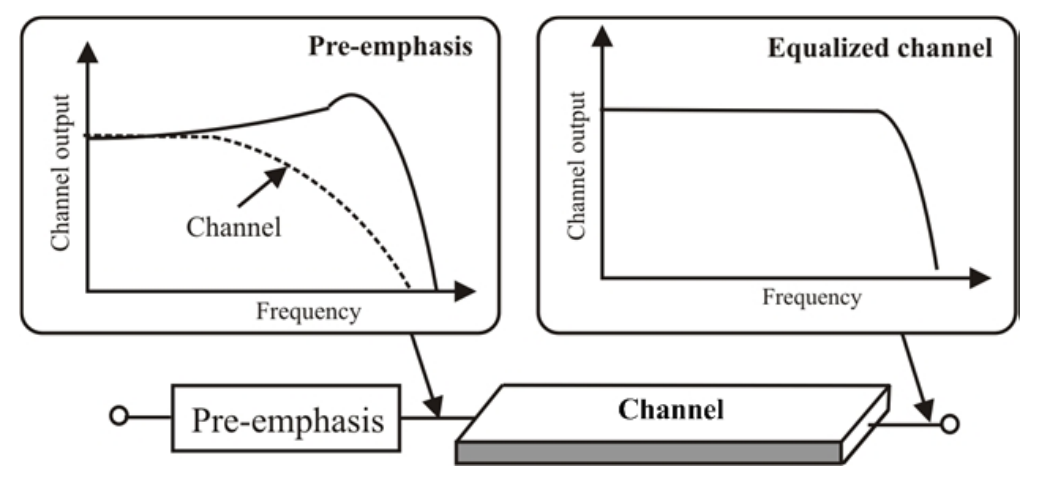

Figure 1.6: Frequency response of an equalized channel with pre-emphasis. The high-frequency components of data symbols are boosted prior to their transmission.

\section{De-Emphasis Equalization}

De-emphasis equalization is the other technique falls within the classification of pre-emphasis. The principle of this technique is depicted in Fig.1.7. The low-frequency components of the transmitted symbol are attenuated while keeping the high-frequency components intacted. The received symbol at the channel far end is flattened. De-emphasis reduces the total amount of the transmitted-symbol-power subsequently deteriorates the bit-error-rate (BER) of the data link. This is because the power of the data symbol is largely concentrated at low frequencies. 


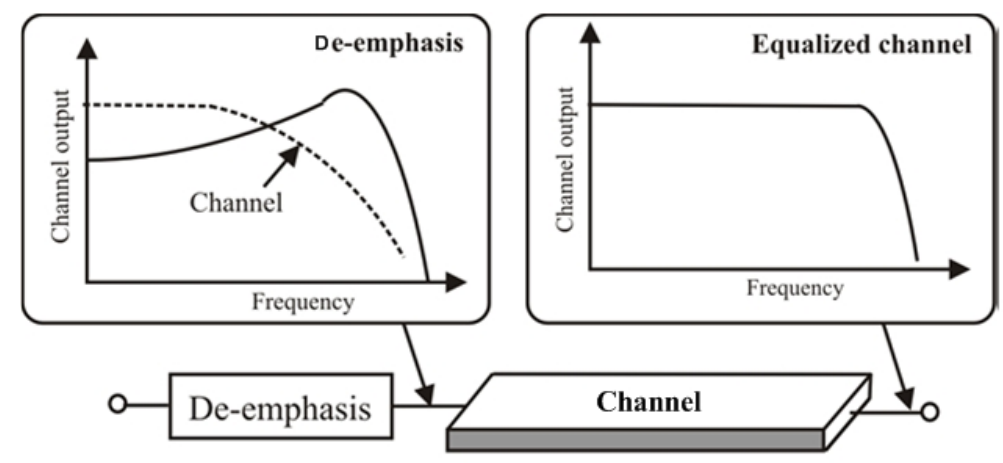

Figure 1.7: Frequency response of an equalized channel using de-emphasis. The low-frequency components of data symbols are attenuated prior to their transmission.

\subsubsection{Far-End Channel Equalization}

Far-end channel equalization also known as post-equalization combats ISI by either amplifying the high-frequency components of received data symbols in the analog domain or removing post-cursors in the digital domain prior to clock and data recovery (CDR). As compared with near-end equalization, post-equalization offers the ability to combat ISI caused by reflection and crosstalk.

\section{Linear Post-Equialization}

Linear post-equalization boosts the high-frequency components of received data-symbols with a continuous-time linear equalizer (CTLE). CTLE provides zeros to cancel out the poles of the channels so that the equalized channel exhibits an all-pass transfer characteristic. To demonstrate this, consider the CTLE in Fig.1.8 and neglect the capacitance of MOSFETs. We examine three cases :

- If only $C_{x}$ is considered (neglect $R_{x}, L$, and $C_{L}$ ), the transfer function is given by 


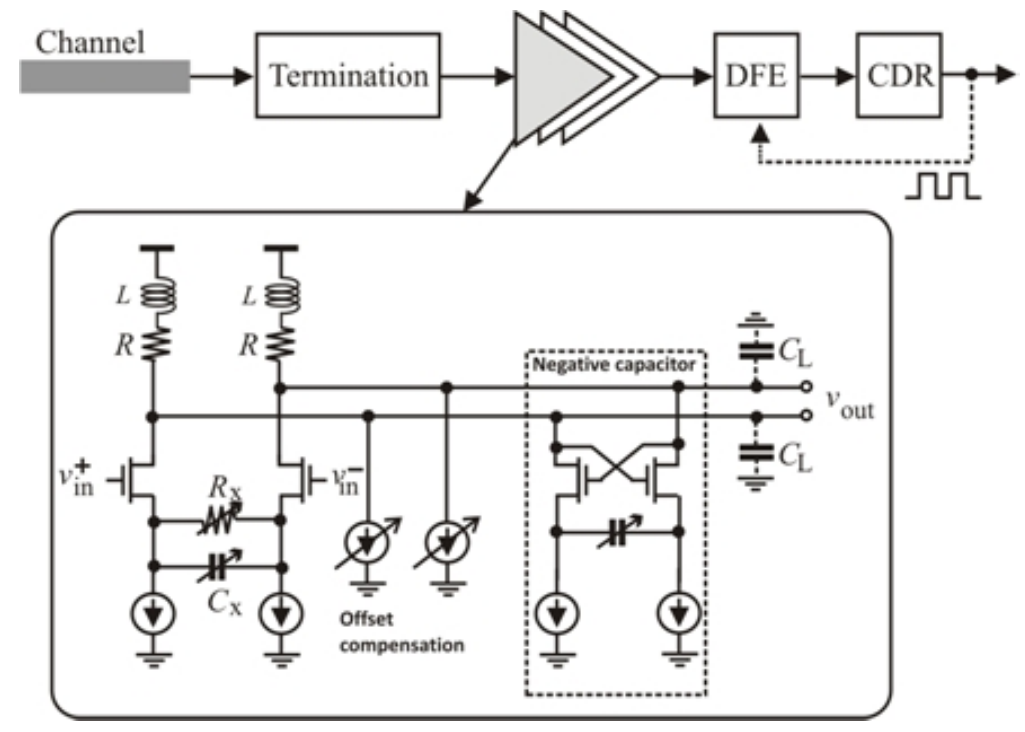

Figure 1.8: Continuous-time linear equalizers with inductor series peaking, source degeneration, and negative capacitors.

$$
\frac{V_{o}(s)}{V_{\text {in }}(s)}=-\frac{s R C_{x}}{\frac{s C_{x}}{g_{m}}+1},
$$

where $g_{m}$ is the transconductance of the MOSFETs. The feedback provided by $C_{x}$ adds a zero at frequency $\omega_{z}=0$. The pole provided by $C_{x}$ is at frequency $\omega_{p}=g_{m} / C_{x}$. $\omega_{p}$ must be sufficiently higher than the half baud-rate frequency so that its impact is negligible. The domain in which the added zero is effective in compensating the effect of the poles of the channel is given by $\omega_{z} \leq \omega \leq \omega_{p}$.

- If we consider both $C_{x}$ and $R_{x}$ (Neglect $L$ and $C_{L}$ ), the transfer function becomes

$$
\frac{V_{o}(s)}{V_{i n}(s)}=-\frac{R g_{m}\left(s R_{x} C_{x}+1\right)}{\left(R_{x} g_{m}+1\right)\left(\frac{s R_{x} C}{R_{x} g_{m}+1}+1\right)},
$$


The zero is now located at $\omega_{z}=1 /\left(R_{x} C_{x}\right)$ and the pole is at $\omega_{p}=1 /\left(R_{x} g_{m}+\right.$ 1)/( $\left.R_{x} C_{x}\right) \approx g_{m} / C_{x}$ provided $R_{x} g_{m}>>1$. It is evident that $\omega_{z}$ is now tunable by varying $C_{x}$ and $R_{x}$.

- If $L, R_{x}, C_{x}$, and $C_{L}$ are all considered, the transfer function becomes

$$
\frac{V_{o}(s)}{V_{i n}(s)}=-\frac{R g_{m}\left(s R_{x} C_{x}+1\right)\left(\frac{s L}{R}+1\right)}{\left(R_{x} g_{m}+1\right) L C_{L}\left(\frac{s R_{x} C}{R_{x} g_{m}+1}+1\right)\left(s^{2}+\frac{s L}{R}+\frac{1}{L C_{L}}\right)},
$$

It is seen from Equation(1.5) that the addition of the inductor peaking introduces another zero at $\omega_{z 2}=R / L$. This is in addition to the zero introduced by $C_{x}$ and $R_{x}$ at $\omega_{z 1}=1 /\left(R_{x} C_{x}\right)$. It also introduces complex conjugate poles with natural resonant frequency $\omega_{n}=1 / \sqrt{L C_{L}}$. It is well understood that complex conjugate poles improve bandwidth [16]. The zeros are used to cancel the effect of the poles of the channel so as to increase the bandwidth while the complex conjugate poles improve the bandwidth through resonance. The higher the quality factor, the larger the bandwidth improvement. The addition of the negative capacitors reduces $C_{L}$, which in turn boosts the natural resonant frequency $\omega_{n}$ subsequently the bandwidth.

The use of zeros to offset the effect of the poles of wire channels bears a strong resemblance to the use of filtering mechanisms to compensate for the loss of wireless channels so as to shorten channel impulse response length or equivalently improve the channel bandwidth, for example, the time truncation of channel impulse response by filtering proposed in [17]. The computational cost of these mechanisms, however, makes them difficult to meet the ever stringent timing constraints of multi-Gbps serial links. 
As the received symbol is severely attenuated by the channel upon arriving CTLE, input offset voltage compensation is also required in CTLE [66]. The order of CTLE is determined by the attenuation of the channel and the sensitivity of the slicer. High-order CTLE can be obtained by cascading low-order CTLEs at the cost of more power consumption [19]. CTLE is often used in conjunction with nonlinear post-equalization with the former providing secondary channel equalization. As a result, low-order DFE can be used without sacrificing performance [20]. CTLE has also been used as a solo post-equalizer for channels with negligible reflection and cross-talk. The absence of feedback in this case allows CTLE to support higher data rates. For example, CTLE in 130 nm CMOS enables 10 Gbps transmission over 30 FR4 channel of $-21 \mathrm{~dB}$ loss at half baud-rate frequency and achieves $10^{-13}$ BER [21]. Similarly, CTLE implemented in $130 \mathrm{~nm}$ CMOS supports 10 Gbps transmission over 34 FR4 channel with $-14 \mathrm{~dB}$ loss and consumes $6 \mathrm{~mW}$ [22]. It should be emphasized that CTLE is only effective in removing channel loss-induced ISI and ineffective in eliminating crosstalk/reflection-induced ISI [21].

\section{Nonlinear Post-Equalization}

Non-linear equalization is another technique of an equalization that is used at the channel far-end prior to a clock and data recovery operation. It uses past decisions rather than amplifying the high-frequency components or attenuating the low-frequency components of the received symbol to mitigate ISI in the time domain. Decision feedback equalization DFE technique is the most widely used nonlinear equalization at the far end of the channel.

\subsection{Decision Feedback Equalization}

Nonlinear post-equalization compensates the high frequency loss of the channel by removing the long tail of the received symbol in the time domain. The most widely used non-linear equalization is the decision feedback equalization (DFE) introduced by Austin in 1967 with its basic configuration shown in Fig.1.9 [23]. 


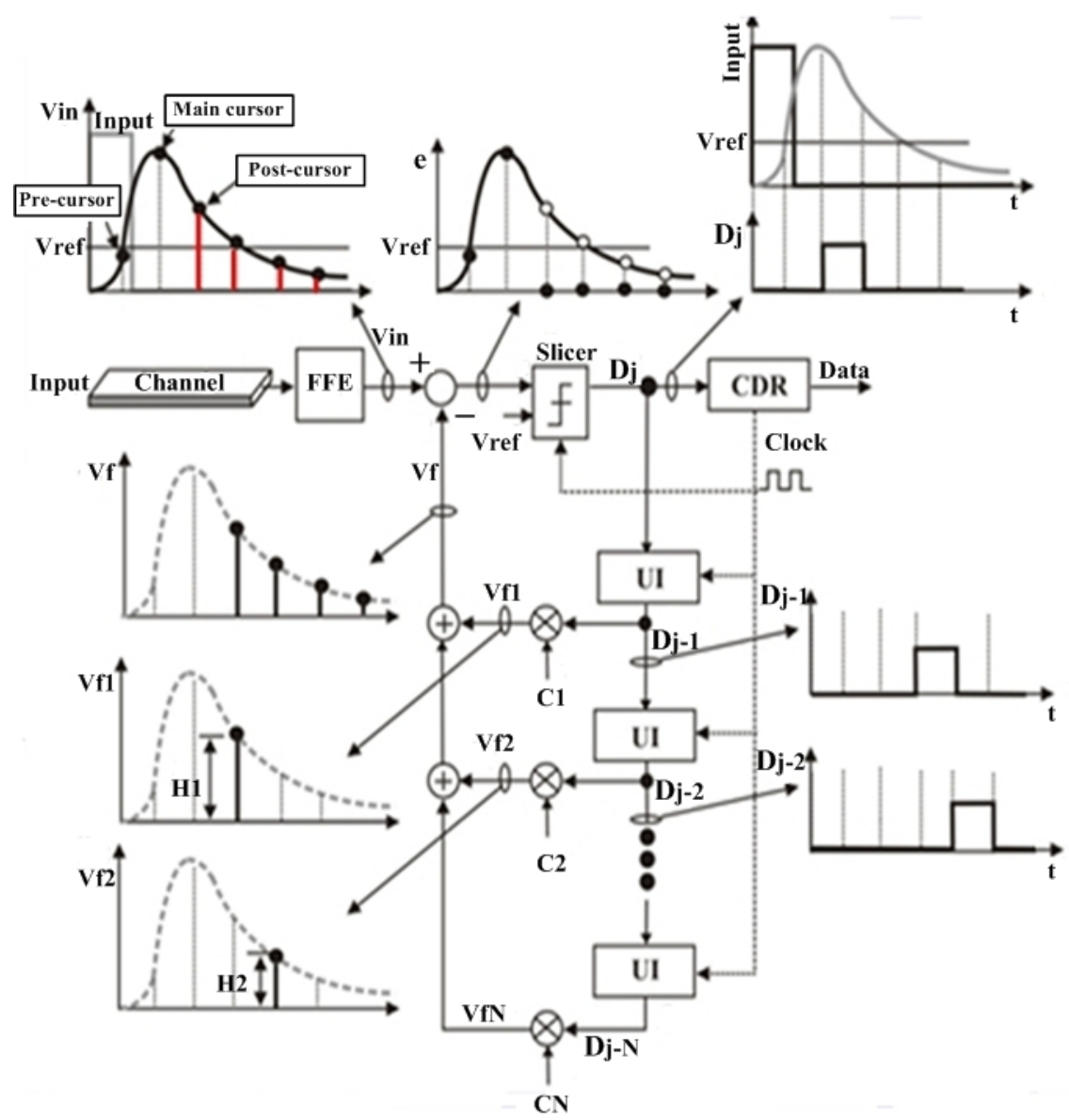

Figure 1.9: Basic configuration and operation of decision feedback equalization. Legends: UI Delay cell with one unit delay, $v_{s}=v_{i n}-v_{f}$ is the symbol before the slicer. $v_{r e f}$ is the threshold of the slicer, $D_{j=1}$ is the delayed version of $D_{j}, c_{1}, \ldots, c_{N}$ are the weighting factors of feedback taps, $T_{s}$ is the symbol time, $H_{j, k}$ denote the $k$ th post-cursor of symbol- $j$ and $H_{0, j}$ the main cursor of symbol- $j$.

The voltage comparator known as data slicer is clocked by the recovered clock. It samples the difference between the current symbol and the feedback signal, and makes a Boolean decision based on the comparison. To minimize the error in data slicing, the received symbol needs to be sufficiently large. This is achieved by amplifying the received symbol prior to slicing [20]. Because the amount of amplification needed in forward equalization at the far- 
end of the channel is set by the sensitivity of the data slicer, which is typically much smaller as compared with that required for channel equalization with a linear equalizer at the near end of the channel, the cross-talk induced by feed-forward equalization at the receiver is minimal. The output of the slicer passes through $N$ delay stages where $N$ is the number of the post-cursors of the current symbol. The output of each delay stage is multiplied by a proper weighting factor $c_{k}$ such that $H_{k}=c_{k} D_{j-k}$ holds where $H_{k}$ is the $k^{\text {th }}$ post-cursor of the current symbol and $D_{j-k}$ is the $(j-k)^{t h}$ past decision of the slicer. It becomes evident that the functionality of the feedback network of DFE is to replicate the post-cursors of the current symbol using the past decisions such that when the feedback is subtracted from the current symbol, the post-cursors of the current symbol are removed ideally.

To illustrate how DFE removes the post-cursors of the current symbol in an intuitive way, let us assume that the current and previous symbols at the far-end of the channel have a main cursor, and four post-cursors. Further, let us assume that there are consecutive logic-1s and the response of the channel to logic-1s is identical, i.e., the response of the channel has the same main cursor and post-cursors. Assume symbol-1 has been correctly detected and DFE is now processing the next symbol. The output of the data slicer for symbol-1 is delayed by one unit interval (1UI), typically one symbol time, with a delay cell and then multiplied by a proper weighting factor $c_{1}$ to create $H_{1}=c_{1} D_{j-1}$ where $H_{1}$ is the amplitude of the first post-cursor of the current symbol such that when subtracted from the current symbol, the first post-cursor of the current symbol is removed, as illustrated graphically in Fig.1.9. To remove the remaining post-cursors, an additional three delay cells and multipliers are needed. Mathematically, if we let symbol $j$ be $v_{i n, j}$, then in the discrete domain, it can be written as

$$
v_{i n, j}=v_{j}+\sum_{k-1}^{4} v_{j-k}+n_{j}
$$


where $v_{j}$ denotes the main cursor of symbol $j, v_{j-k}, k=1,2,3,4$ denote the four postcursors of symbol $j$, and $n_{j}$ denotes the noise present in the symbol and sampled at the time when the main cursor is sampled. Note that $v_{j-k}=0$ if $i<k$. The output of the feedback network is given by

$$
v_{f, j}=\sum_{k-1}^{4} c_{k}+D_{j-k}
$$

where $D_{j-k}, k=1,2,3,4$ are the four past decisions of the slicer and $c_{k}$ is the weighting factor assigned to $D_{j-k}$. The input of the slicer is given by

$$
\begin{gathered}
e_{j}=v_{i n, j}-v_{f, j}, \\
e_{j}=v_{j}+\sum_{k-1}^{4}\left[v_{j-k}-c_{k} D_{j-k}\right]+n_{j} .
\end{gathered}
$$

It becomes clearly that if $c_{k}$ is properly chosen, the term in the brackets in (1.9) will vanish, i.e., the post-cursors of the symbol will be completely removed. Also observed is that DFE operation has no impact on the noise present in the current symbol. Since DFE restores square-wave-like symbols by removing the post-cursors, it is equivalent to boosting the highfrequency components of the received symbol in the frequency domain. Although DFE has no effect on the precursors of the current symbol, fortunately ISI is primarily caused by post cursors. DFE is proven to be an effective, robust, and perhaps the most widely used technique to combat ISI of Gbps data links. 


\subsection{Chapter Summary}

The imperfections of wire channels and their impact on data transmission were investigated. The pros and cons of near-end and far-end channel equalizations that combat ISI were explored. A detailed examination of the principle, configuration, operation, and limitation of DFE was provided.

\subsection{Contributions of The Dissertation}

The contributions of this dissertation are:

1. A comprehensive review of DFE for multi-Gbps data links is conducted. The review examines: i) The imperfection of wire channels, in particular, finite bandwidth, reflection, and cross-talk, and their impact on data transmission. ii) The fundamentals of both near-end and far-end channel equalization to combat the effect of the imperfection of wire channels at high frequencies. iii) Design challenges encountered in design of DFE for multi-Gbps data links including timing constraints, sampling, error propagation, arithmetic operation, highly dispersive channels, power consumption, and techniques and circuit implementations that address these challenges. iv) The need for adaptive DFE, the principles of adaptive DFE, the performance of various adaptive DFEs and their pros and cons.

2. A two-dimensional hexagon eye-opening monitor (EOM) for Gbps serial links is proposed. As compared with rectangular EOM, the proposed EOM provides a tightened control of data jitter at the edge of data eyes and eliminates unnecessary errors flagged by the rectangular EOM. Moreover, it is able to recognize the severity of the errors. This allows the adaptive engine to provide variable step-size for updating DFE tap coefficients and reduces the convergence time of the adaptation process. 
3. A power-efficient two-dimensional on-chip EOM for Gbps data links is proposed. The proposed EOM employs the half pattern of hexagon and rectangle EOMs to detect the violation of received data signals with the minimum required detection at the clock and data recovery (CDR) operation. As compared with full hexagon and rectangular EOMs, the proposed EOM eliminates half of the required comparators while keeping the same accuracy of error detection.

4. A hexagon EOM-based variable step-size adaptive DFE for multi-Gbps data links is proposed. The proposed adaptive DFE utilizes a half-hexagon EOM to detect the error of the received data signals. The step size used in search for the optimal tap coefficients of DFE is set by the severity of the violation of the pre-defined minimum eye-opening by received data symbols so as to achieve both a fast convergence of adaption and the maximum eye-opening. The effectiveness of the proposed adaptive DFE is evaluated by embedding it in a 1 Gbps serial link implemented in an IBM $130 \mathrm{~nm}$ 1.2V CMOS technology. For the purpose of comparison, an adaptive DFE with a rectangular EOM is also designed and included in the same data link. Simulation results demonstrate that the proposed adaptive DFE maximizes the eye-opening of received data symbols. Also, it outperforms the adaptive DFE with a rectangular EOM by reaching the convergence approximately 4 times faster.

5. A jitter-based adaptive decision feedback equalizer for high-speed serial links is proposed. The adaptation in search for the optimal tap coefficients of DFE is set by the detection of the violation of the maximum allowable jitter by the received data.

\subsection{Organization of The Dissertation}

The dissertation is organized in 7 chapters. Chapter 1 provides a comprehensive review of decision feedback equalization for multi-Gbps data links. In the chapter, we examine the effect of iner-symbol interference on the integrity of received data signal, the imperfections of wire channels, in particular, finite bandwidth, reflection, and cross-talk, and their impact on 
data transmission. Also, in this chapter, we reveal a detailed examination of the principle, configuration, operation, and limitation of DFE. Design challenges encountered in design of DFE for Gbps data links and techniques and circuit implementations that address these challenges address in Chapter 2. Chapter 3 proposes a two-dimensional hexagon EOM for Gbps data links. We show that the proposed EOM outperforms diamond and rectangular EOMs for a better error detection and shorter adaptive time. A power efficient hexagon EOM is presented in Chapter 4. Chapter 5 presents an adaptive decision feedback equalizer utilizing the proposed hexagon EOM for detecting errors. In Chapter 6, a maximum jitter based decision feedback equalizer is proposed. Chapter 7 concludes the dissertation and outlines future works. 
Table 1.1: Data rate of serial links utilizing decision feedback equalization. Channel loss is measured at half baud-rate frequency.

\begin{tabular}{|c|c|c|c|c|c|c|}
\hline Ref. & Tech. & Channel loss & Data rate & $\mathrm{Tx}$ & $\mathrm{Rx}$ & BER \\
\hline [68] & $130 \mathrm{~nm}$ & $-8 \mathrm{~dB}$ & $3.7 \mathrm{Gbps}$ & - & 3 IIR & $10^{-12}$ \\
\hline$[34]$ & $130 \mathrm{~nm}$ & $-21 \mathrm{~dB}(36 " \mathrm{FR} 4)$ & 6.25 Gbps & - & 5-tap & $10^{-15}$ \\
\hline$[30]$ & $130 \mathrm{~nm}$ & -18 dB (33” FR4) & 9.6 Gbps & 2-tap & CTLE/1-tap & $10^{-15}$ \\
\hline [11] & $130 \mathrm{~nm}$ & $-18 \mathrm{~dB}$ (30” FR4) & $6.4 \mathrm{Gbps}$ & 4-tap & 5 -tap & $10^{-15}$ \\
\hline$[15]$ & $130 \mathrm{~nm}$ & $-12 \mathrm{~dB}(26 " \mathrm{FR} 4)$ & 5 Gbps & - & 1-tap & - \\
\hline$[20]$ & $130 \mathrm{~nm}$ & $-(40 "$ FR4 $)$ & $6.4 \mathrm{Gbps}$ & 2-tap & 4-tap & $2 \times 10^{-15}$ \\
\hline$[31]$ & $90 \mathrm{~nm}$ & $-6.2 \mathrm{~dB}(10 " \mathrm{SMA})$ & $6.0 \mathrm{Gbps}$ & - & 2-tap & $10^{-12}$ \\
\hline$[45]$ & $90 \mathrm{~nm}$ & -12 dB (16" Тусо) & 7.0 Gbps & - & 2-tap & $10^{-13}$ \\
\hline [69] & $90 \mathrm{~nm}$ & $-(18 " \mathrm{BP})$ & -7.5 Gbps & - & 10-tap & $10^{-12}$ \\
\hline [29] & $90 \mathrm{~nm}$ & -33 dB (16" FR4) & 10 Gbps & 4-tap & 5-tap & $10^{-12}$ \\
\hline [66] & $90 \mathrm{~nm}$ & $-25.4 \mathrm{~dB}(29 " \mathrm{FR} 4)$ & 10.3 Gbps & 2/3-tap & CTLE/1-tap & $10^{-13}$ \\
\hline$[70]$ & $90 \mathrm{~nm}$ & $-25.4 \mathrm{~dB}(15 " \mathrm{FR} 4)$ & 10 Gbps & - & 2-tap & $10^{-12}$ \\
\hline$[71]$ & $90 \mathrm{~nm}$ & $-32.7 \mathrm{~dB}(5.5 " \mathrm{FR} 4)$ & 6 Gbps & - & 1-tap \& IIR & $10^{-12}$ \\
\hline$[72]$ & $90 \mathrm{~nm}$ & -14 dB (20" Nelco) & 15 Gbps & - & 1-tap & $10^{-13}$ \\
\hline$[75]$ & $65 \mathrm{~nm}$ & $-16 \mathrm{~dB}(30 " \mathrm{PCB})$ & 11 Gbps & 3-tap & 5-tap & $10^{-15}$ \\
\hline [25] & $65 \mathrm{~nm}$ & $-21 \mathrm{~dB}(50 "$ Nelco $)$ & 10 Gbps & - & 1-tap \& IIR & $10^{-12}$ \\
\hline [19] & $65 \mathrm{~nm}$ & $-24 \mathrm{~dB}(28 " \mathrm{FR} 4)$ & 8.5 Gbps & - & 3-tap & - \\
\hline [58] & $65 \mathrm{~nm}$ & $-24 \mathrm{~dB}(12 " \mathrm{PCB})$ & 12.5 Gbps & 4-tap & 2-tap FFE/5-tap & - \\
\hline$[56]$ & $65 \mathrm{~nm}$ & $-13.3 \mathrm{~dB}(34 " \mathrm{FR} 4)$ & 5 Gbps & - & 1-tap & $10^{-12}$ \\
\hline [74] & $65 \mathrm{~nm}$ & $-13.3 \mathrm{~dB}(34 " \mathrm{FR} 4)$ & $5 \mathrm{Gbps}$ & - & 1-tap & $10^{-12}$ \\
\hline$[76]$ & $65 \mathrm{~nm}$ & $-13.3 \mathrm{~dB}(16 " \mathrm{FR} 4)$ & 21 Gbps & 3-tap & 1-tap & $10^{-12}$ \\
\hline$[42]$ & $65 \mathrm{~nm}$ & $-22.3 \mathrm{~dB}(14 " \mathrm{FR} 4)$ & 20 Gbps & - & CTLE/1-tap & $10^{-12}$ \\
\hline$[77]$ & $45 \mathrm{~nm}$ & $-21 \mathrm{~dB}(50 "$ Nelco $)$ & 10 Gbps & - & DFE-IIR & $10^{-12}$ \\
\hline [78] & $45 \mathrm{~nm}$ & $-25 \mathrm{~dB}(18 " \mathrm{FR} 4)$ & 15 Gbps & - & 2-tap & $10^{-12}$ \\
\hline$[79]$ & $45 \mathrm{~nm}$ & $-32 \mathrm{~dB}$ (40" Nelco) & 16 Gbps & 3-tap & 12-tap & $10^{-15}$ \\
\hline$[80]$ & $45 \mathrm{~nm}$ & $-25 \mathrm{~dB}(20 " \mathrm{PCB})$ & 19 Gbps & - & 4-tap FFE/5-tap & $10^{-13}$ \\
\hline [81] & $40 \mathrm{~nm}$ & $-10 \mathrm{~dB}(3 " \mathrm{FR} 4)$ & 16 Gbps & - & 1-tap & $10^{-12}$ \\
\hline [82] & $40 \mathrm{~nm}$ & -15 dB (3" FR4) & 20 Gbps & 1-tap & CTLE/1-tap & $10^{-12}$ \\
\hline [83] & $40 \mathrm{~nm}$ & $-34 \mathrm{~dB}(24 "$ FR4) & 16 Gbps & 3-tap & 14-tap & $10^{-12}$ \\
\hline [84] & $40 \mathrm{~nm}$ & $-20 \mathrm{~dB}(8 " \mathrm{FR} 4)$ & 23 Gbps & 2-tap & CTLE/1-tap & $10^{-12}$ \\
\hline$[85]$ & $32 \mathrm{~nm}$ & $-25 \mathrm{~dB}(14 " \mathrm{PCB})$ & 11.8 Gbps & 3-tap & 4 -tap & $10^{-12}$ \\
\hline [86] & $32 \mathrm{~nm}$ & $-27 \mathrm{~dB}(39 ” \mathrm{PCB})$ & 12.5 Gbps & - & CTLE/8-tap & $10^{-12}$ \\
\hline [87] & $32 \mathrm{~nm}$ & $-35 \mathrm{~dB}(15 " \mathrm{PCB})$ & 28 Gbps & 4-tap & CTLE/15-tap & $10^{-13}$ \\
\hline [88] & $28 \mathrm{~nm}$ & $-33 \mathrm{~dB}$ BP & 12.5 Gbps & 5-tap & CTLE/3-tap & $10^{-15}$ \\
\hline
\end{tabular}




\section{Chapter 2}

\section{Design Challenges in DFE}

This chapter provides design challenges encountered in the design of DFE for multi-Gbps data links including timing constraints, sampling, error propagation, arithmetic operation, highly dispersive channels, power consumption, and techniques and circuit implementations that address these challenges. In the chapter, we investigate the need for adaptive DFE and the principles of adaptive DFE. Finally, we addres the performance of various adaptive DFEs and their pros and cons.

\section{$2.1 \quad$ Bit Error Rate Test}

The performance of serial links is primarily quantified by Bit Error Rate (BER) obtained by transmitting a Pseudo-Random Bit Stream (PRBS) to the channel and recording the number of transmission errors, typically $B E R=10^{-12}$ is required. Transmission errors are obtained using a PRBS checker that compares the transmitted bits with the corresponding received bits. Although PRBS7 (7-bit PRBS) has been used [25], they are primarily for testing serial links with 8B/10B encoded data. PRBS31 (31-bit PRBS) that provides a sufficient transition density is preferred especially for those using 64B/66B encoded data. PRBS can be generated using Linear Feedback-Shift Registers (LFSR), although parallel PRBS generators are also available [26]. Since the data eye-opening is typically maximized at the center of the data eye where BER is minimized and gradually levels off towards the edges of the data 
eye where BER climbs, the horizontal eye-opening at a given BER, for example $10^{-8}$ [27], $10^{-9}[25]$ or $10^{-10}$ [86], is usually used as a figure-of-merit to quantify the performance, as shown in Fig.2.1. The bathtub curves are obtained by varying the sampling instant within One Unit Interval (One UI) while evaluating BER for each sampling instant [29]. It is seen that BER is minimized at the center of the data eye and gradually levels up when sampling instant moves away from the center towards the edge of the data eye.

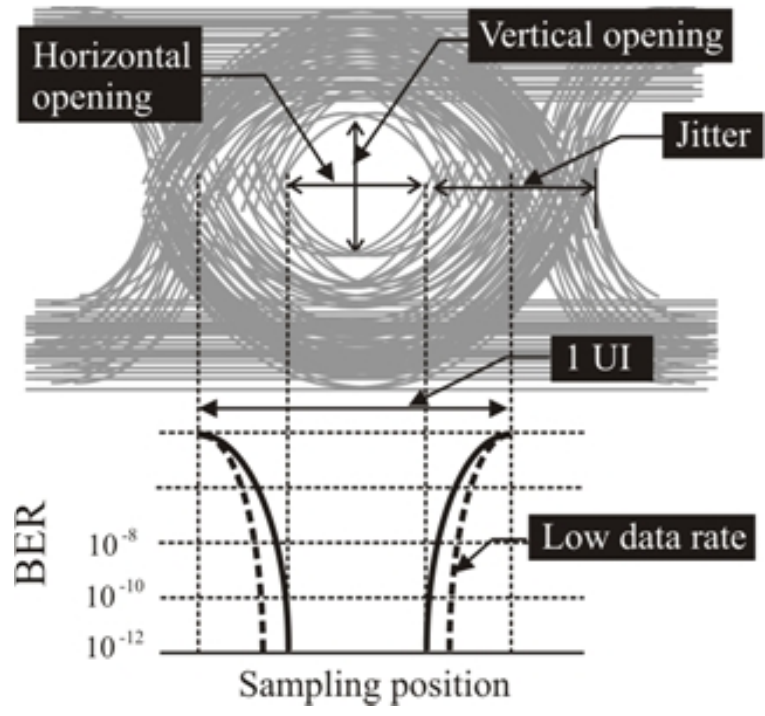

Figure 2.1: Dependence of horizontal eye-opening on BER.

\subsection{Timing Constraints}

The operation performed by DFE including the delay of the previously recovered data, the multiplication of the recovered data by an appropriate weighting factor, the subtraction of the feedback signal from the current symbol, and data slicing must be completed in one UI, i.e., before the arrival of the next symbol. Since there is only one UI between the arrival of the current symbol and tap-1 feedback is allowed, tap-1 delay loop bears most of the timing 
constrain. It becomes increasingly difficult to complete delay, multiplication, subtraction, and data slicing operations within one UI when data rate is high. An effective way to overcome this difficulty is to feed the error signal, i.e., the difference between the signal from the preceding forward equalizer and the feedback signal, to two identical slicers that are in parallel and operated simultaneously, for possible feedback signals, i.e. $H_{1}$. As shown in Fig.2.2, the decisions of the slicers are then multiplexed by a 2-to- 1 multiplexer with the select signal the previous decision [30].

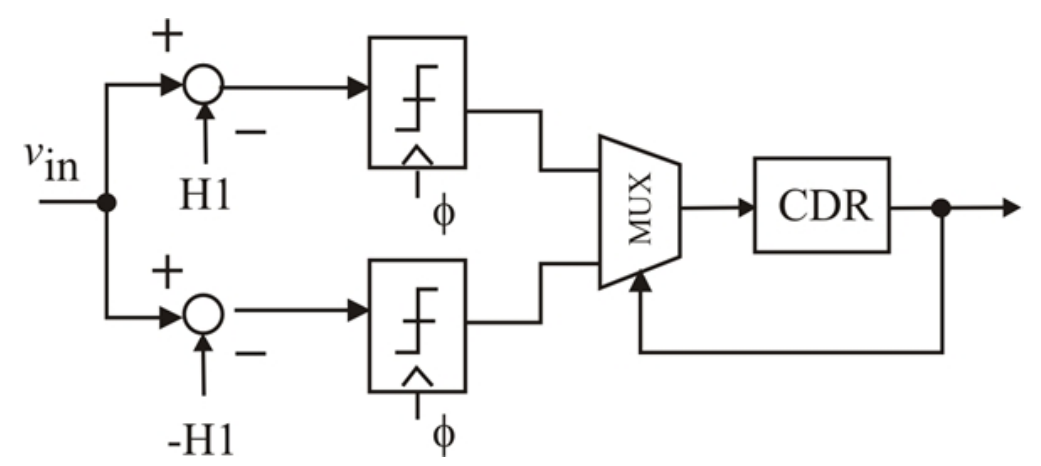

Figure 2.2: Tap-1 feedback is implemented using loop-unrolling.

This approach was originally proposed by Kasturia et al. [31] and is known as loop unrolling, speculation, look-ahead [32], or partial-response [33]. Since the multiplication, subtraction, and slicing can be conducted without waiting for the delay and the delay of 2-to-1 multiplexing is small, the stringent timing constrain on tap-1 feedback loop is greatly relaxed. The use of loop unrolling, however, is typically limited to tap-1 only simply because the number of slicers increases exponentially with the number of taps.

As pointed out in [34], the delay of the regenerative-configured slicer will be overly long once its input is small. To reduce the delay of the slicer, the insertion of an auxiliary amplifier between the forward equalizer and the slicer is proven to be beneficial. The speed gained from the insertion of the auxiliary amplifier overwhelms the delay of the auxiliary amplifier itself. To relax timing constraint and lower power consumption, the half-rate ap- 
proach where all units of the DFE are operated at only half the data rate is widely favored, as depicted graphically in Fig.2.3. Two identical DFE paths are driven by a non-overlapping clock whose frequency is only half that required for the data rate as the two paths operate in an interleaved manner [11]. The relaxed timing constraint not only greatly simplifies design but also lowers power consumption. The timing constraint can be further relaxed using a quarter-rate approach with further reduced power consumption at the cost of power and silicon consumption [31].

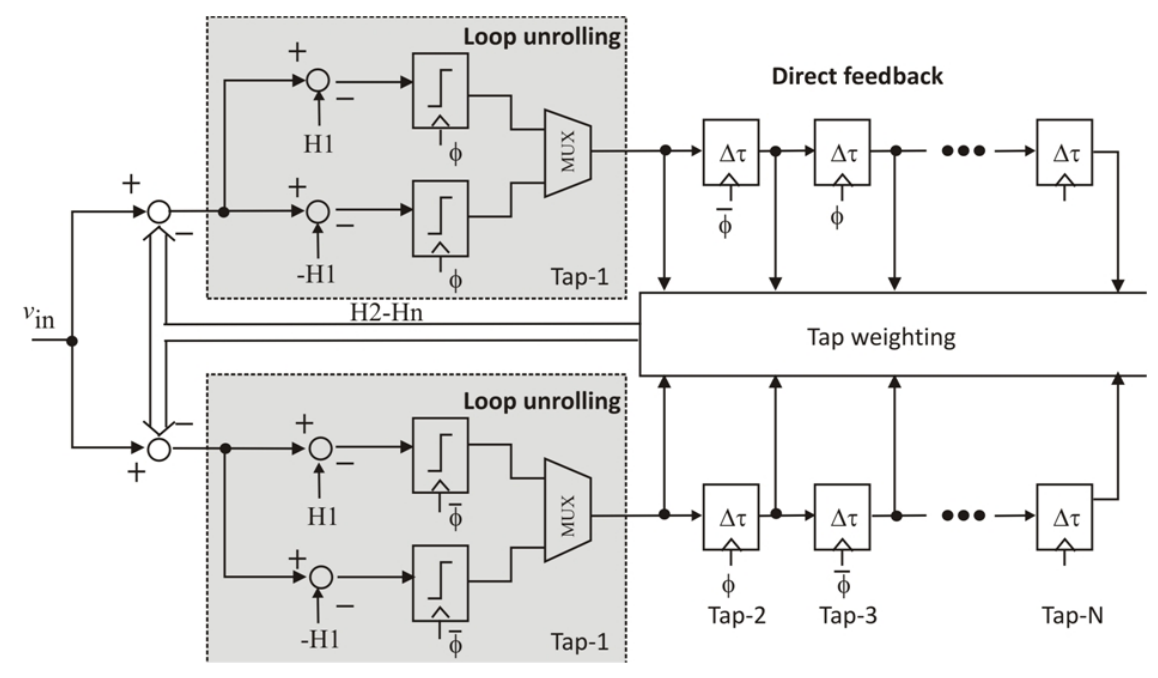

Figure 2.3: Half-rate decision feedback equalization. Tap-1 is implemented using loop-unrolling while the remaining taps are implemented using direct feedback. Note that complementary clocks are used for upper and lower paths of DFE.

\subsection{Timing Alignment}

The removal of the post-cursors of the current symbol is achieved by subtracting the weighted past decisions from the current symbol. This approach is effective only if the characteristics of the channel does not change over $N$ consecutive UIs, where $N$ is the number of the taps of DFE. If there exists a timing alignment error between the post-cursors of the current symbol and the feedback signal, the post-cursor will not be removed completely even though 
the amplitude of the feedback signal is identical to that of the post-cursor, as illustrated graphically in Fig.2.4 where a DFE with 2 taps is shown. As can be seen that the timing error of the delay stages $\Delta T_{s}$ where $T_{s}$ is the symbol time, i.e. $T_{s}=\mathrm{UI}$, will give rise to a
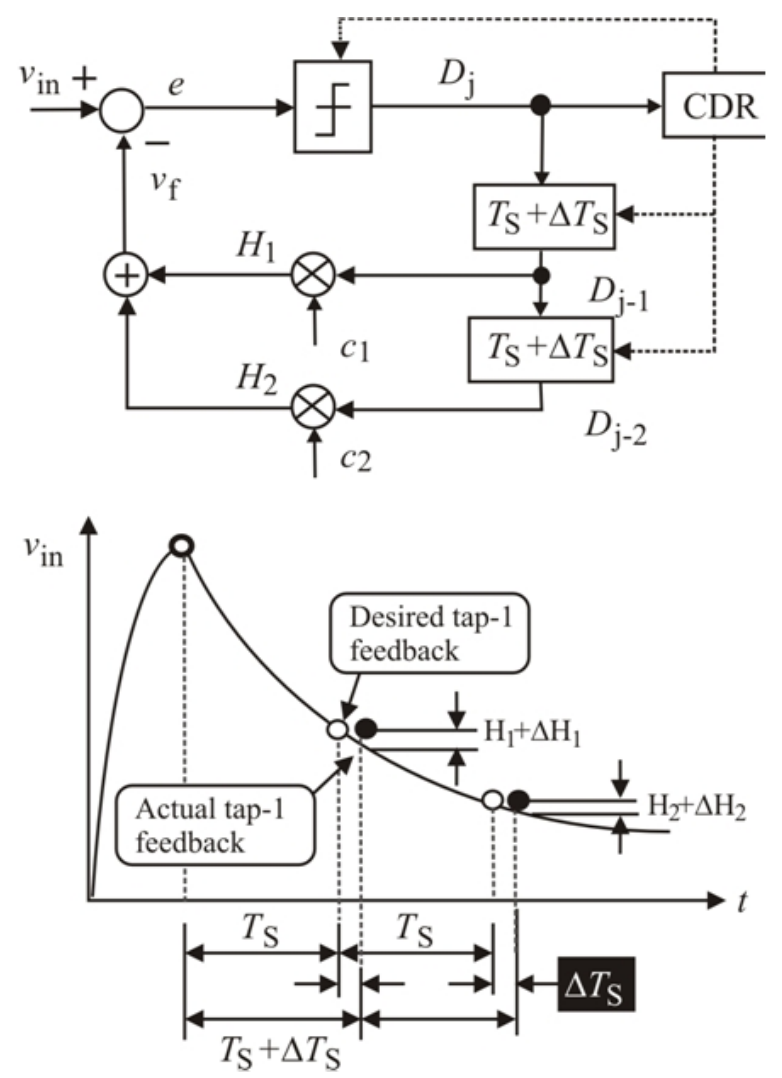

Figure 2.4: Post-cursor cancellation error due to timing error of delay stages.

$$
\Delta H_{k} \approx\left[\frac{\partial v_{i n}}{\partial t}\right]_{t=k T_{s}} \Delta T_{s}
$$

feedback error $\Delta H_{k}$. When subtracted from the current symbol, $\Delta H_{k}$ of the $k$ th postcursor of the current symbol will remain. Clearly $\Delta H_{k}$ is directly related to $\Delta T_{s}$ and the profile of the impulse response of the channel.

Since the feedback signal passes through a train of delay stages whose delay is subject to 
the effect of process spread, voltage fluctuation, and temperature variation (PVT), a timing alignment error between the incoming signal and the DFE feedback at the input of the slicer will exist. One solution for this is to clock all delay stages, as shown in Fig.2.3. This, however, might become difficult when data rate is high. Also, the delay of $v_{f}$ consists of the delay of the delay stages and that of the summer. The delay of the summer is not controlled by the clock.

In [6], a variable delay block whose input is the recovered clock and whose output controls the delay stage and the operation of the summer of the DFE was used. The delay of the delay stage is adjusted in a training phase. A training sequence is used prior to the normal operation to allow the receiver to adjust the delay of the delay blocks such that the timing error between the input and the DFE feedback is minimized.

\subsection{Error Propagation}

If an erroneous decision is made by the slicer, for example, the slicer outputs a logic-0 even though it supposes to output a logic-1, the summer will subtract a weighted decision from the current symbol even though it supposes to add the weighted decision to the current symbol. Clearly the error will impact the next decision of the slicer. The error of the slicer will also propagate through the delay chain and affect the remaining DFE operations, as illustrated in Fig.2.5 where the response of a channel with 1 post-cursor is used to demonstrate the effect of the error of data slicing on the removal of the post-cursors. This error propagation characteristic of DFE is an intrinsic drawback of DFE -based channel equalization. To minimize the possibility of slicer errors, at least three approaches are at our disposal. The first is to sample the incoming signal multiple times and the correct decision is made from majority voting [35]. This approach, though effectively, might become difficult and also costly when data rate is high. Second, the error of the data slicer can also be reduced using current- integrating where the incoming signal is integrated over a capacitor and the resultant capacitor voltage is sampled at the end of integration phase [36]. Current-integration essentially forms 
a low-pass filter capable of filtering out spikes whose duration is much smaller as compared with UI. For disturbances with a large duration, its effectiveness diminishes. Finally, the error of the slicer can be minimized if the incoming signal is sufficiently large.

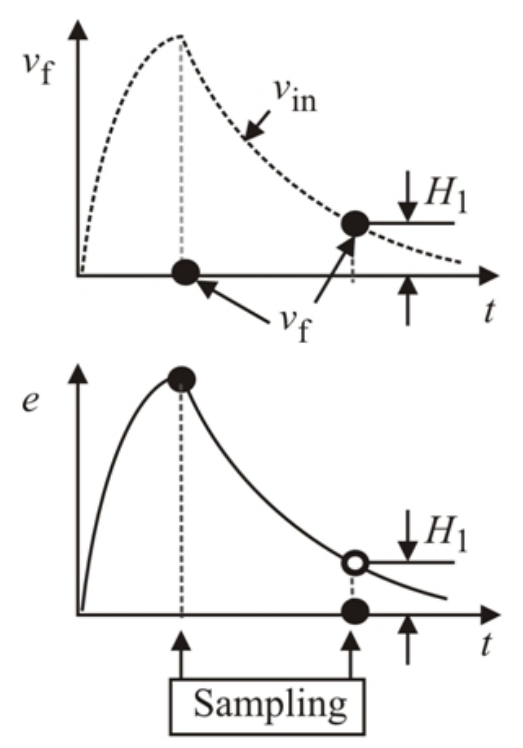

(a) No error in data slicing

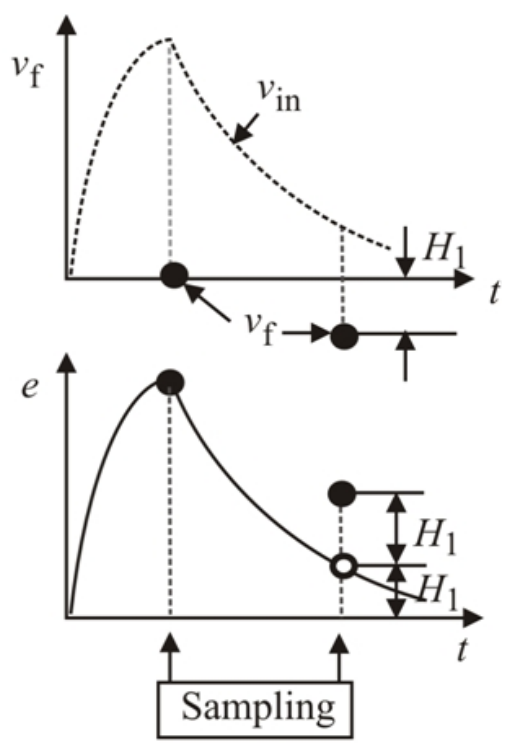

(b) Error in data slicing

Figure 2.5: Error in data slicing affects the removal of post-cursors.

This can be achieved by pre-amplifying the signal at the far-end of the channel prior to slicing, i.e. feed-forward equalization.

\subsection{Calibration of Slicer}

The slicer is typically implemented using a re-generative configuration where a pair of crosscoupled inverters is used for speed improvement during latching and noise rejection when latch is established, as shown in Fig.2.6 [37]. When $\phi=0$, the regenerative mechanism is disabled and the input and output of the cross-coupled inverters are set to be equal, driving the operating point of the inverters to the transition region where its voltage gain is 
maximized. The input and reference voltage, in the mean time, are sampled by the input capacitors of the slicer. In the following phase where $\phi=1$, the re-generative mechanism is activated and the voltage sampled in the previous phase is sensed by $M_{2}-M_{5}$.

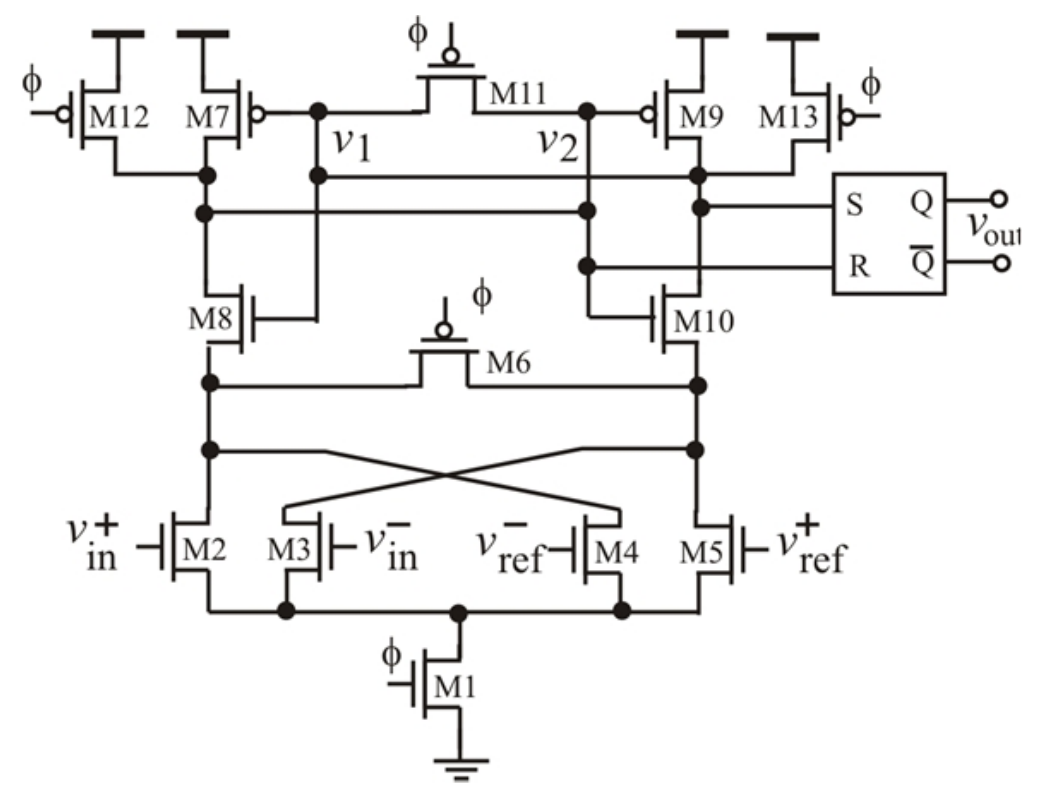

Figure 2.6: Clocked re-generative sense amplifier as slicer [37].

Depending upon the polarity of $v_{i n}-v_{r e f}$, the output of the slicer will be set. The regenerative mechanism ensures that the delay of the slicer is minimized. Transistor $M_{6}$ ensures that when closed, the identical transistors $M_{2}$ and $M_{5}$ will have the same input capacitance. $M_{11}$, when closed, forces the cross-coupled inverters to set their operating point to the transition region where a maximum voltage gain exists. Transistors $M_{12}$ and $M_{13}$, then closed, force the output of the slicer to be at logic-1.

As pointed out earlier that post-cursor elimination is critically affected by the correctness of the decision of the slicer. If the slicer makes an erroneous decision, the erroneous decision will propagate down the delay chain and affect other feedback taps. Whether the slicer will make a correct decision or not largely depends upon the threshold sensing of the input signal. Clearly a safe margin between the input and the threshold of the slicer is therefore needed 
in order to minimize the error of the slicer.

Since the signal at the far end of the channel is severely attenuated due to the loss of the channel, the input offset voltage of the slicer must be sufficiently small in order for the slicer to pick up the severely attenuated signal and make a correct decision. It was shown in [66], the BER of a slicer with an input offset voltage $V_{\text {os }}$ is given by

$$
\mathrm{BER} \approx \frac{1}{2} \operatorname{Err}\left(\frac{V_{m}-V_{o s}}{\sqrt{\overline{V_{n}^{2}}}}\right)
$$

where $\bar{V}_{n}^{2}$ is the input-referred noise power, $V_{p p}$ is the peak-to-peak voltage swing of the input, and $\operatorname{Err}(x)=1 / \sqrt{2 \pi} \int_{x}^{\infty} e^{-u^{2} / 2} d u$ is the error function. The input offset voltage directly affects the BER. Large input devices are preferred from a low input offset voltage point of view as the mismatch-induced input-voltage offset is inversely proportional to the dimension of the transistors [39]. Small input devices, on the other hand, are favored from a low input capacitance subsequently a high operation speed point of view, however, at the cost of deteriorating input offset voltage. This configuration works well with the half-rate DFE depicted earlier. The calibration of the slicer, specifically, the removal of the effect of input offset voltage, prior to any data-slicing operation is inarguably mandatory. In [30], a background calibration method was used to calibrate slicers. Specifically, two identical slicers are employed in parallel. The input of one slicer is connected to the input for sensing (online slicer) while that of the other slicer is connected to a reference voltage for calibration (off-line slicer) [15]. The on-line slicer and the off-line slicer are operated in an interleaved manner such that the slicer that is online is always properly calibrated. The calibration of the offline slicer can be accomplished using conventional auto-zero techniques to remove the effect of the input offset voltage. The effect of the input offset voltage of the slicer can also be compensated by using the dynamic offset control technique proposed in [40] to avoid the power penalty of the current array-based offset compensation [41] and the speed penalty of 
capacitor array-based offset compensation [42]. In [43], a novel digital offset compensation technique was proposed for limiting amplifiers of optical communications. The method detects the effect of the offset voltage on the duty-cycle of the output and utilizes the detected duty-cycle imbalance to adjust the biasing currents so as to eliminate the duty-cycle imbalance subsequently the offset effect. The method avoids the deployment of compensation capacitors at the input of the limiting capacitors subsequently its detrimental impact on speed.

\subsection{Delay Cells}

Delay cells play a critical role in DFE. The performance of delay cells is governed by three design constrains: shortest delay due to ever reduced UI, delay tunability, and power consumption. The propagation delay of delay cells is UI. This constrain excludes familiar static logic delay cells.

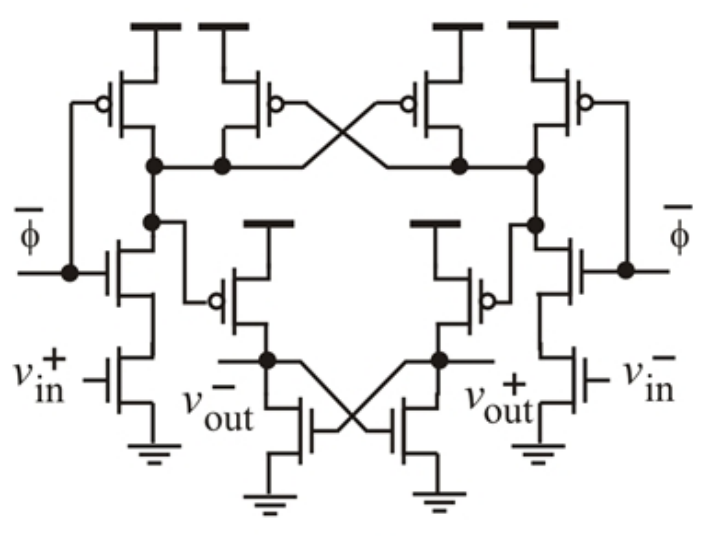

(a)

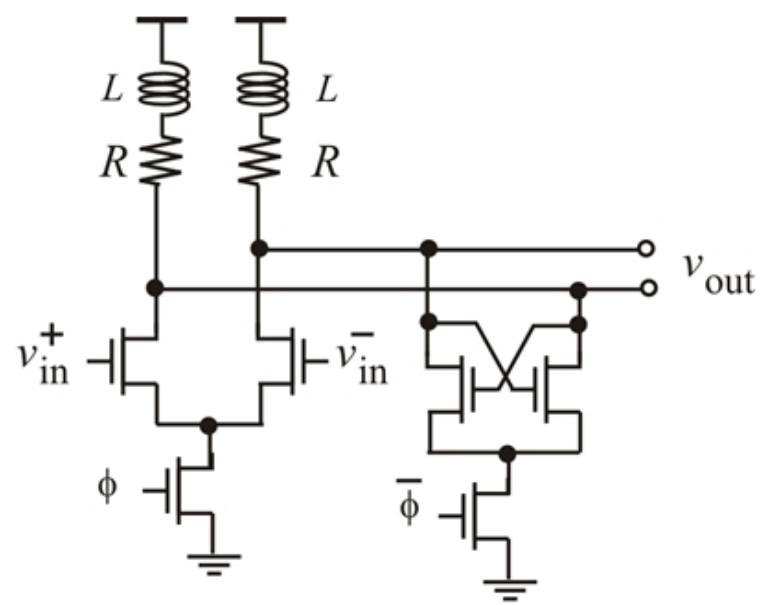

(b)

Figure 2.7: (a) Clocked re-generative sense amplifier as delay cell [25], (b) Clocked current-mode delay cells [44].

High-speed delay cells and techniques typically used for speed enhancement such as inductor 
peaking and re-generation are widely used in design of delay cells. Delay tunability is needed to ensure that the feedback and the signal from the preceding forward equalizer are in phase so that a proper subtraction of the feedback from the current symbol can be executed to remove the post-cursors of the current symbol. Any timing error between the feedback and incoming signal will undermine the effectiveness of DFE, as depicted in detail earlier in Section 2.3. Minimization of the power consumption of delay cells is also critical especially when the large number of delay cells is used. Current-mode delay cells with a tunable propagated delay are typically preferred, however, at the expense of static power consumption. Fig.2.7 (a) is a delay stage that uses two-stage re-generation to reduce the time delay [25]. Fig.2.7 (b) is a current-mode delay cell with both re-generation and inductor shunt peaking for delay reduction [44].

\subsection{Arithmetic Operation}

Both multiplication and summation operations are needed in DFE. Not only these operations must be completed within one UI, the result must be stable prior to any slicing operation. Multiplication of a past Boolean decision by a weighting factor is most efficiently implemented using current-steering configurations, as shown in Fig.2.8.

The delay of the summer usually dominates the speed of arithmetic operation due to the large number of feedback taps [11]. Current mode summation is the most widely favored over its voltage mode counterpart due to its ease of implementation and high-speed operation. The speed of the current-mode resistor-load summer shown in Fig.2.8 (without inductors) is set by the time constant of the current summation node. Since $V_{i n}$ is attenuated, the transistors driven by $V_{i n}$ are operated in saturation whereas those in the tap stages are operated in an $\mathrm{ON} / \mathrm{OFF}$ mode. Lowering the load resistance $\mathrm{R}$ reduces the time constant, however, at the cost of reduced output voltage swing. As the output of the summer directly feeds the slicer, a large output voltage of the summer is essential to minimize slicer error. Increasing the dimension of the input transistors improves output voltage swing, it, however, 
also reduces the speed. One effective way to speed up the summer without reducing the load
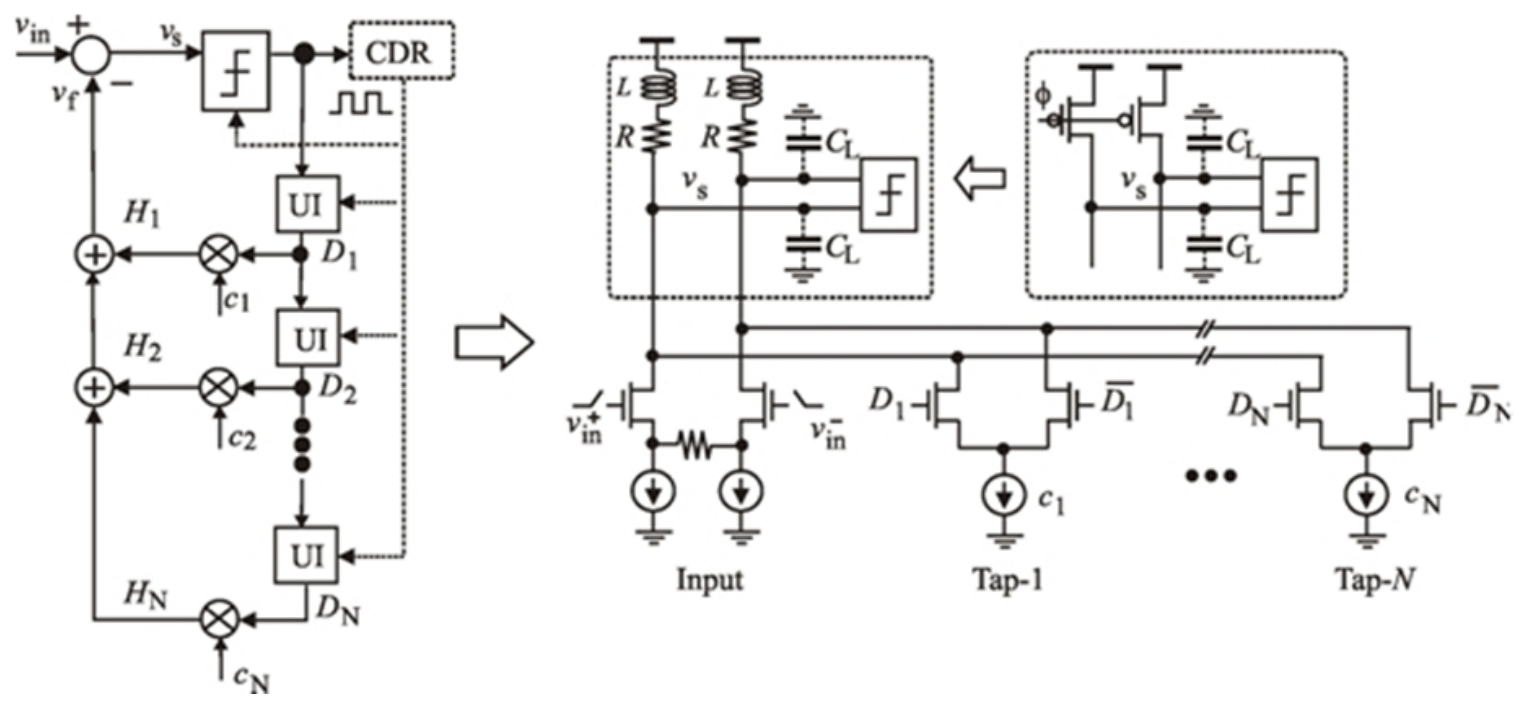

Figure 2.8: Current-mode summer with resistive load.

resistance is shunt inductor peaking, as shown in Fig.2.8 [42]. In [45], a current-integrating summer shown in Fig.2.8 was proposed. To improve speed, source degeneration is widely adopted. The load resistors are replaced with PMOS transistors operated in an ON/OFF mode. In the reset phase, PMOS transistors are switched on and the load capacitors are charged to the supply voltage. During the following integration phase, PMOS transistors are switched off and the capacitors are discharged by the tail current sources representing the feedback taps. To eliminate the effect of $V_{i n}$ during discharge, $V_{i n}$ is disconnected from the gate of the input transistors [5]. The current-integrating summer offers the key advantage of reduced power consumption because there is no static current flowing from VDD to ground in both the reset and integrating phases [27]. The speed of the current-integrating summer can be further increased by replacing current feedback taps with capacitive charge feedback, as shown in Fig.2.8, with capacitance proportional to DFE tap coefficients [86]. To further reduce power consumption, switched-capacitor summers were proposed [46]. Since complex clock schemes are needed for their proper operation, switched-capacitor summers are typically used to perform the summation of the first-tap with the rest of the taps implemented 
using the current-integrating approach depicted earlier.

\subsection{Channels with Severe Dispersity}

The impulse response of severely dispersive channels stretches over a large number of symbol intervals, as seen in Fig.1.2(d). To equalize these channels, a large number of taps is needed, resulting in excessive power and silicon consumption. Efficient DFE with a small number of taps without sacrificing performance is highly desirable.

DFE with an analog IIR (Infinite-Impulse-Response) filter uses an analog IIR filter to mimic the response of the channel such that when subtracted from the response of the channel, the tail is removed without using a large number of DFE taps [25]. This approach works well for highly dispersive channels.

The characteristics of channels with severe reflection, however, differ from those of channels with high loss but insignificant reflection. The impulse response of these channel typically have post-cursors that reside far away from the main cursors. The post-cursors between the dominating post-cursors typically immediately following the main cursor and reflectioninduced post-cursors are often insignificant, leading to sparsity in post-cursor distribution. Although there are many effective means to equalize sparse wireless channels [47], these approaches cannot be adopted for wire channels due to the need for excessive computation subsequently long latency. Equalization of these channels requires a long fixed-tap DFE even though many of the taps corresponding to the insignificant post-cursors between the main cursors and the remotely placed post-cursors are insignificant, resulting in excessive power and silicon consumption. Floating-tap DFE proposed by Zhong et al. is an elegant technique effective in combating reflection-induced post-cursors located far away from the main cursor [4]. In this approach, a number of fixed-taps are used to remove dominant post-cursors located close to the main cursor. In addition, a number of floating-taps whose locations are not fixed but rather determined by an optimization algorithm that yields the largest tap coefficients subsequently the best performance are used to remove reflection-induced post- 
cursors. Although extra computation is needed, this additional cost is well justified by the elimination of the remote post-cursors.

\subsection{Power Consumption}

The power consumption of a decision feedback equalizer consists of the power consumption of the slicer, the delay units, and the summer. The power consumption of the summers is significant due to their current-mode configuration. When loop-unrolling is employed, additional power consumption exists. The DFE-IIR examined earlier offers an attractive means to reduce power consumption, especially for highly dispersive channels. In [55], a soft-decision DFE was proposed to replace loop-unrolling and dynamic feedbacks without sacrificing speed. Instead of employing two slicers and other logic circuits, soft-decision DFE uses sample-and-hold before the summation and latches after the summation to perform channel equalization.

\subsection{Adaptive Decision Feedback Equalization}

DFE reduces the post-cursors of the current symbol by adjusting the tap coefficients of the DFE FIR filter so that the subtraction of the feedback from the current symbol will result in the complete elimination of its post cursors. The choice of the optimal number of the taps of the FIR filter and the coefficient of each tap should therefore be made in the consideration of the required BER and power consumption of the data link. The variation of the characteristics wire channels requires an adaptive DFE where the tap coefficients of DFE are set automatically in accordance with the characteristics of the channels. Although adaptive DFE for low-speed wire-line communications such as telephony and cable TVs has been studied extensively, adaptive DFE for Gbps data links over wire channels and their silicon realization are still in its infancy. A number of novel algorithms, architectures, and silicon implementations of adaptive DFE for Gbps data links emerged recently. In this section, we 
examine the adaptive DFE algorithms that are widely used for Gbps serial links.

\subsubsection{Least-Mean-Square Adaptive DFE}

Least mean square (LMS) adaptation updates the DFE tap coefficients in such a way that the power of the error between the output and input of the slicer is minimized, i.e. minimize $\left\|D_{j}-v_{s, j}\right\|$, as shown graphically in Fig.2.9 and architecturally in Fig.2.10.

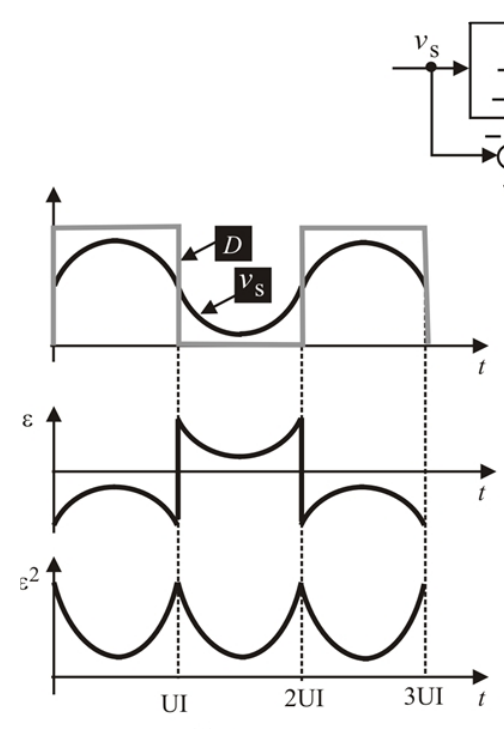

(a) Large error

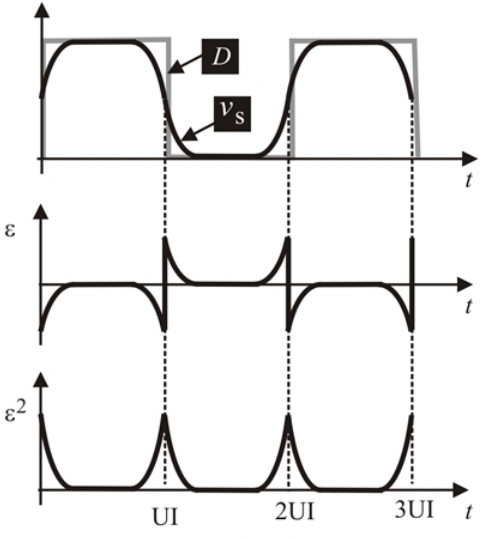

(b) Small error

Figure 2.9: Least-mean-square adaptive DFE.

The tap coefficients $c_{k}$ in step $k$ of DFE are updated using [48]

$$
c_{j, k+1}=c_{j, k}+h \epsilon_{k} v_{k-j},
$$

where $h$ is the step size used to adjust the tap coefficients. LMS is difficult to implement 


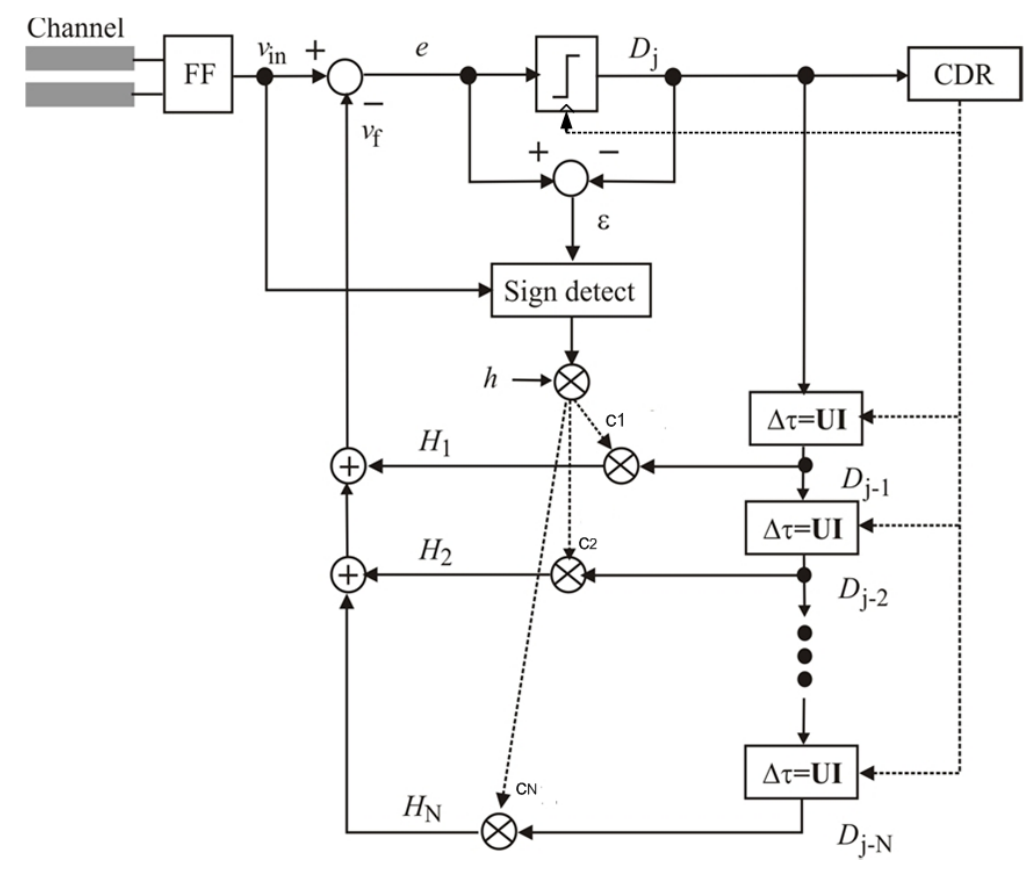

Figure 2.10: Configuration of SS-LMS adaptive DFE.

due to the need for the value of $\epsilon_{k}$ and signal $v_{k-j}$ that can only be obtained using ADCs. Sign-sign LMS (SS-LMS) where only the sign of $\epsilon_{k}$ and $v_{k-j}$ are used is proven to be an effective alternative

$$
c_{j, k+1}=c_{j, k}+h \operatorname{sign}\left(\epsilon_{k}\right) \operatorname{sign}\left(v_{k-j}\right),
$$

$\operatorname{sign}\left(\epsilon_{k}\right)$ and $\operatorname{sign}\left(v_{k-j}\right)$ can be obtained conveniently using slicers. Since SS-LMS searches for the optimal tap coefficients based on the binary decision of the sign of $\epsilon_{k}$ and $v_{k-j}$, the final optimal value of the tap coefficients will fluctuate in the vicinity of the optimal taps. In practice, a smaller $h$ is typically used by SS-LMS to reduce the fluctuation. The convergence time of SS-LMS, however, will be shorter as compared with regular LMS. 


\subsubsection{Eye-Opening Adaptive DFE}

The opening of data eyes reflects the effect of the imperfections of the channel over which data are transmitted. It is a measure of the quality of the data link and can therefore be used to guide the search for the optimal parameters of DFE to maximize eye-opening. The optimal tap coefficients should result in the maximum eye opening [34]. The quality of a data eye is quantified by a number of parameters such as vertical eye-opening, horizontal eye-opening, and eye-edge jitter, as shown in Fig.2.11.

The eye-opening of the signal at the input of the slicer can be captured using an onchip eye-opening monitor (EOM). On-chip eye-opening monitors can be loosely classified into (i) 1-dimensional eye-opening monitors, (ii) 2-dimensional eye-opening monitors, (iii) data edge-based eye-opening monitors, and (iv) multi-sampling eye-opening monitors. A 1-dimensional eye-opening monitor quantifies the opening of data eyes by either the vertical or horizontal dimension of the eye with the vertical opening the most widely used due to the ease of measurement [49]. The underlining principle of 1-dimensional EOM- based adaptive $\mathrm{DFE}$ is that the input of the slicer is compared with two reference voltages representing the desired vertical eye opening at the sampling instant by an error sampler [53]. The sign of the result of the comparison is used to adjust DFE tap coefficients so as to maximize the eye opening, as shown in Fig.2.12. They are the simplest in term of hardware and consume the lowest power. The horizontal eye-opening can be determined from oversampling the received data symbol. This, however, is at the cost of high silicon and power consumption [27].

Improvements were made in [50] where both the edges and the center of the data eye are used to quantify the opening of the data eye. A 2-dimensional monitor quantifies the eyeopening by measuring the dimension of the eye in both the vertical and horizontal directions [51]. This is the most widely used eye-opening monitors and required moderate hardware and power. Data edge-based eye-opening monitors quantify the eye-opening by examining the edges of data eyes (zero-crossing) using multiple samples [52]. They are power consuming. Multi-sampling eye-opening monitors scan data eyes both vertically and horizontally with a large number of samples per bit time. They are most comprehensive but at the highest cost 


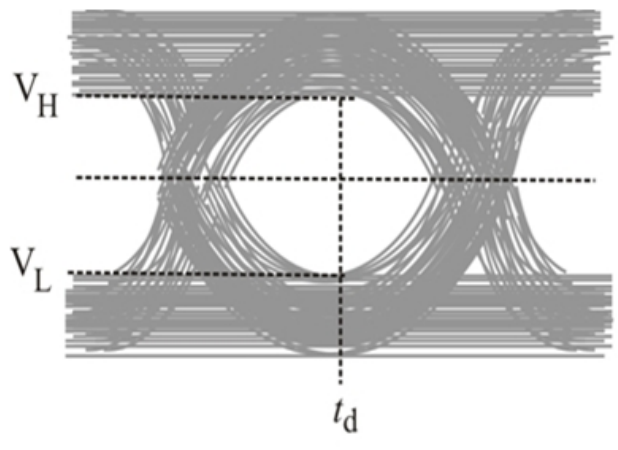

(a) 1-dimensional EOM

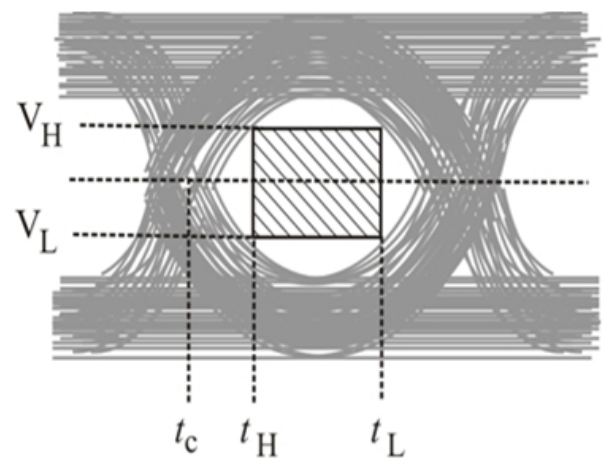

(b) 2-dimensional EOM

Figure 2.11: Eye-opening monitors.

of silicon and power.

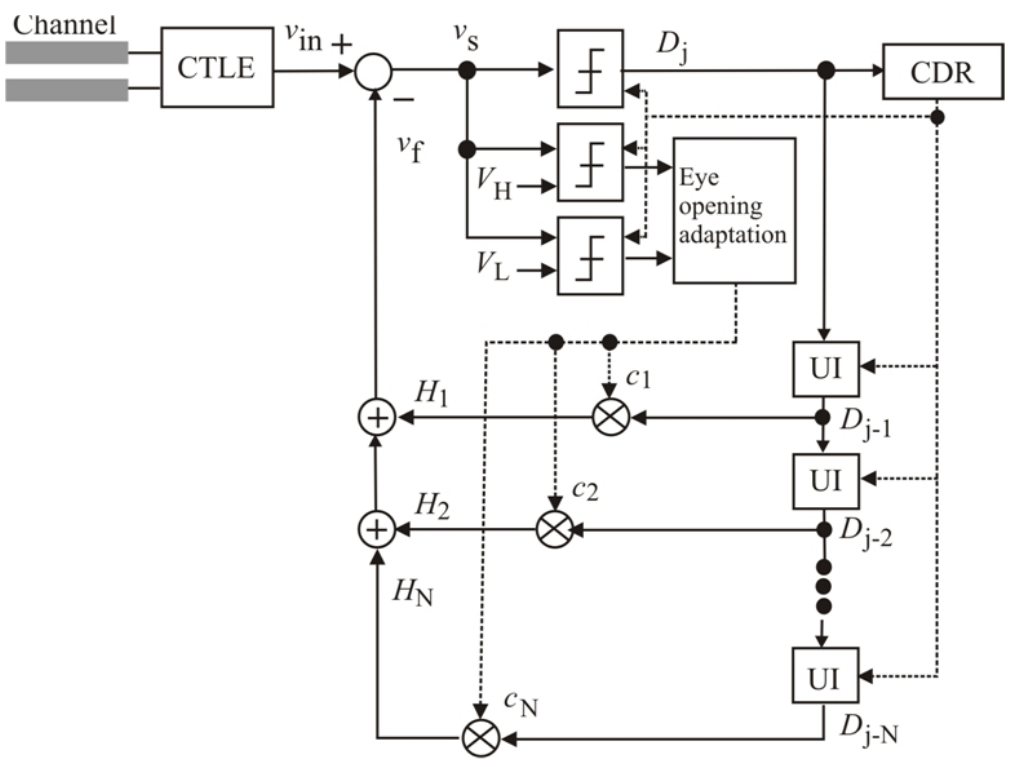

Figure 2.12: 1-dimensional Eye-opening adaptive DFE [53].

\subsubsection{Jitter-Based Adaptive DFE}

The intrinsic relation between the vertical and horizontal openings of data eyes reveals that minimizing timing jitter at the edges of the eye will also maximize the vertical opening of the 
data eye subsequently, as illustrated graphically in Fig.2.13. To illustrate this, we represent the eye-diagram with zero jitter with a sinusoid $v_{s}(t)=V_{m} \sin \left(\omega_{s} t\right)$ where $\omega_{s}=\pi / T_{s}$, as shown in Fig.2.13. We further assume that the eye-diagram with non-zero jitter is simply the down-shifted version of the one without jitter, i.e. $\hat{v}_{s}(t)=V_{m} \sin \left(\omega_{s} t\right)-\Delta V_{m}$, where $\Delta V_{m}$ is the variation of the amplitude due to timing jitter. It is straightforward to show that $\Delta \tau=T_{s} / \pi \sin ^{-1}\left(\Delta V_{m} / V_{m}\right)$. If $\Delta \tau \ll T_{s}$, we have $\Delta \tau \approx T_{s} / \pi\left(\Delta V_{m} / V_{m}\right)$. It is evident that $\Delta V_{m}$ is directly proportional to $\Delta \tau$. In [54], a jitter-based eye-opening monitor was proposed. The transition edge of data eyes is sampled by a number of samplers, as shown in Fig.2.13. XOR gates are used to determine the location of the transition edges and counters to record the number of transitions at each sampling position.

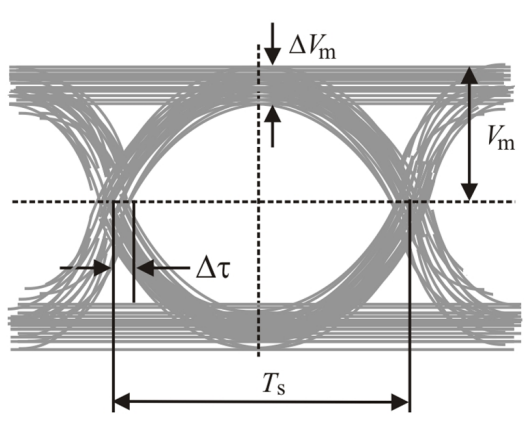

(a) Small eye-edge jitter

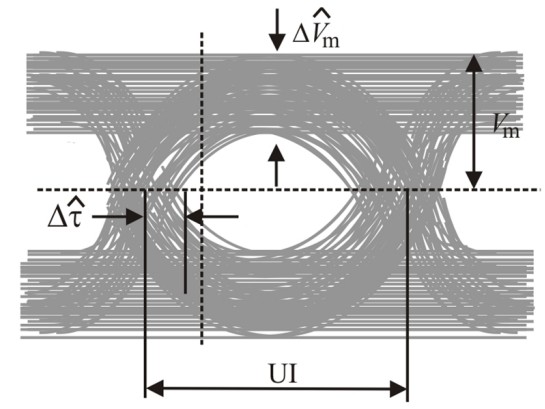

(b) Large eye-edge jitter

Figure 2.13: Relation between vertical opening and edge jitter.

An edge-transition histogram is generated. Measurement results demonstrate that the larger the eye opening, the more narrow the histogram. Since the quality of the obtained histogram depends upon the number of samplers, this method is power hungry. Also, it becomes difficult to employ multiple samplers when data rate is high. To simultaneously minimize the timing jitter and maximize the vertical opening, a dual-mode adaptive DFE was proposed [55]. The dual-mode DFE consists of a data DFE and an edge DFE with the former maximizing the vertical opening and the latter minimizing the timing jitter. The edge adaptive DFE reduces the eye-edge timing jitter by $30 \%$ without sacrificing vertical opening. 


\subsubsection{Comparison}

The preceding presentation of EOM-based adaptive DFE, jitter-based adaptive DFE, and ADC-based adaptive DFE, reveals the following intrinsic advantages of these adaptive DFE as compared with LMS adaptive DFE :

1. EOM and jitter based adaptive DFE allow designers to freely set the constraints with which the optimization algorithms must satisfy. These constraints such as vertical and horizontal eye-openings and timing jitter are directly related to BER of data links.

2. Multiple constraints such as eye-opening and timing jitter at the edges of data eyes can be imposed simultaneously to obtain significantly improved performance, as demonstrated in [55].

3. The constraint of optimization constrain is entirely set by users. For example, in [59], a hexagon two-dimensional EOM was proposed to provide better measurement of the minimum data eye so as to provide an improved two-dimensional EOM adaptive DFE.

4. The step size of EOM adaptive DFE can be set adaptively in accordance with the level of the severity of the violation of the minimum eye-opening or timing jitter so as to provide improved adaptivity and performance, as demonstrated in [49].

\subsection{Chapter Summary}

Design challenges encountered in DFE for multi-Gbps data links including BER, timing constraints, error propagation, arithmetic operation, sampling, and delay cells, and circuit techniques addressing these challenges were studied. The need for and the principle of adaptive DFE were also investigated. 


\section{Chapter 3}

\section{2-Dimensional Hexagon Eye-Opening Monitor}

This chapter presents a new two-dimensional on-chip EOM for Gbps serial links. A review of the state-of-the-art of on-chip EOMs is provided and their pros and cons are investigated. A new hexagon two-dimensional EOM that outperforms the widely used rectangular two-dimensional EOMs is introduced and the implementation details are presented. The effectiveness of the proposed EOM is evaluated by embedding it in a serial link implemented in an IBM $130 \mathrm{~nm}$ 1.2V CMOS technology. For the purpose of comparison, a rectangular two-dimensional EOM is also included in the same data link. The data link with variable channel length and attenuation is analyzed using Spectre from Cadence Design Systems with BSIM 4 device models and results are presented.

\subsection{Eye-Opening Monitors}

Traditionally, the eye diagram of the received data is obtained using a pattern generator at the transmitter to generate a random bit stream and an oscilloscope at the receiver to capture the received data stream. This is costly especially when data rate is high. In addition, it cannot provide real-time feedback signals needed to adjust the parameter of equalizers. Pattern generators have widely been replaced with an on-chip pseudo-random bit-stream generator [60]. Recently, on-chip EOMs capable of providing the eye opening or even the 
complete eye diagram of the received data so as to allow the real-time adjustment of the parameters of channel equalizers to achieve optimal performance emerged [59]. On-chip EOMs can be loosely classified into one-dimensional EOMs, two-dimensional EOMs, jitter-based EOMs, and eye-pattern monitors.

\subsubsection{One-Dimensional EOMs}

A one-dimensional EOM quantifies the quality of data eyes by the vertical dimension of the eye [61]. The voltage at the center of the eye $t_{d}$ is compared with $V_{H}$ and $V_{L}$ quantifying the maximum and minimum vertical openings of the eye respectively, as shown in Fig.3.1 [53]. If $v\left(t_{d}\right)<V_{H}$ and $v\left(t_{d}\right)>V_{L}$, an error i.e. a violation of the minimum vertical opening of the data, is detected. Otherwise, no error is detected. Counters can be used to record the number of errors at the upper and lower boundaries of the eye separately.

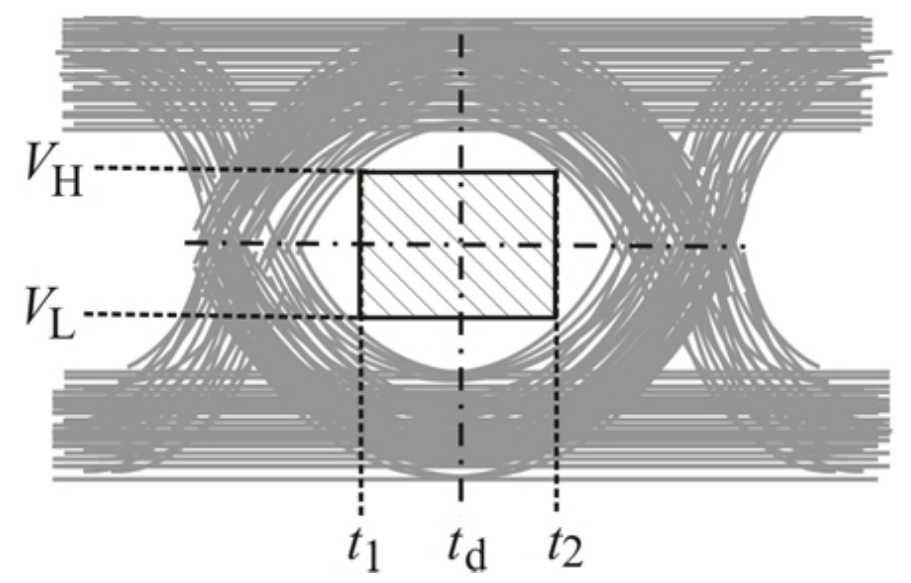

Figure 3.1: One dimensional EOM quantified by $V_{H}$ and $V_{L}$ at $t_{d}$ vertically. Two-dimensional EOM quantified by $V_{H}$ and $V_{L}$ vertically and $t_{1}$ and $t_{2}$ horizontally. 


\subsubsection{Two-Dimensional EOMs}

A two-dimensional EOM quantifies the eye-opening by measuring both the vertical and horizontal dimensions of data eyes, as shown in Fig.3.1 with $t_{1}$ to the left and $t_{2}$ to the right, $V_{H}$ for the maximum voltage, and $V_{L}$ for the minimum voltage [51]. Since $t_{d}$ is determined by clock recovery, $t_{1}$ and $t_{2}$ can be chosen for the desired horizontal opening of the eye by phase-shifting $t_{d}$. $V_{H}$ and $V_{L}$ represent the threshold voltages that are depicted for two comparators at $t_{1}$ and $t_{2}$ to measure the violation of received data symbol with the required detection at the clock and data recovery operation.

\subsubsection{Jitter-Based EOMs}

The preceding two-dimensional EOM uses a rectangular to define the minimum eye opening. It does not take into the consideration of the relation between the vertical opening of the eye and the jitter of the data. Jitter at the edge of the eye reduces the vertical opening of the eye subsequently deteriorates BER, as illustrated graphically in Fig.3.2.
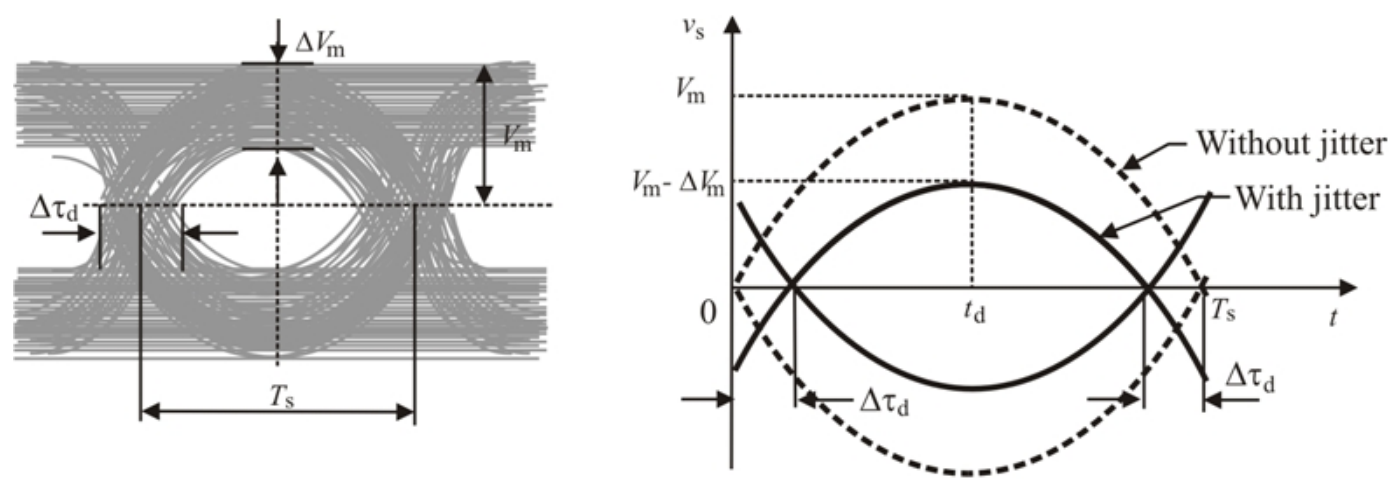

Figure 3.2: Relation between vertical opening and edge jitter.

To find out the relation between the data jitter and the vertical eye-opening, we approximate the waveform of the received symbol of zero data jitter with $v_{s}(t)=V_{m} \sin \left(\omega_{s} t\right)$, as shown 
in Fig.3.2 (the dotted curve). We further assume that the eye-diagram with jitter (the solid curve) is simply the up/down-shifted version of that without jitter (the dashed curve). In this case the received symbol becomes $v_{s}(t)=V_{m} \sin \left(\omega_{s} t\right)-\Delta V_{m}$, where $\Delta V_{m}$ is the variation of the amplitude caused by data jitter $\Delta \tau_{d}$. It is straightforward to show that

$$
\Delta \tau_{d}=\frac{T_{s}}{\pi} \sin ^{-1}\left(\frac{\Delta V_{m}}{V_{m}}\right) .
$$

If $\Delta \tau_{d} \ll T_{s}$, Eq.(3.1) becomes

$$
\Delta \tau_{d} \approx \frac{T_{s}}{\pi}\left(\frac{\Delta V_{m}}{V_{m}}\right) .
$$

It is evident that $\Delta V_{m}$ is proportional to $\Delta \tau_{d}$. The larger the data jitter $\Delta \tau_{d}$, the larger the amplitude variation $\Delta V_{m}$, and the smaller the eye-opening. Jitter-based EOMs indeed can be used to monitor the vertical opening of the eye. Jitter-based EOMs quantify the eye-opening by only examining the data jitter using multiple samples across the edge of the eye without evaluating the vertical opening of the eye [54],[50]. The transition edge of the eye is sampled by a number of samplers and a set of XOR gates with their inputs from two adjacent samplers are used to determine the exact edge of the eye. Counters are used to record the number of transitions at each sampling position and an edge-transition histogram is generated. The larger the eye opening, the narrow the histogram. The histogram provides an effective measure of the eye-opening. A key advantage of this approach that since the detection of the eye edge is also needed in clock recovery operation, the circuitry for clock recovery serve two distinct purposes : recover the clock and monitor eye-opening simultaneously. In [55], a dual-mode adaptive DFE was proposed to simultaneously minimize the jitter 
at the edge of the eye and maximize the vertical opening of the eye. The dual-mode DFE consists of a data DFE and an edge DFE operated independently. The former maximizes the vertical opening whereas the latter minimizes the jitter.

\subsubsection{Eye-Pattern Monitors}

The preceding EOMs cannot provide the information on the actual shape of data eyes. The information is often wanted in the virtual inspection of the eyes. To capture the pattern of the eye, the brute-force approach that samples the data eye from the left edge to the right edge of the eye in a sweeping mode can be employed. The eye-pattern monitor proposed by Noguchi et al. samples the data eye in 128 steps per symbol time [62]. Since multiple sampling clocks are needed, precision phase shifters and phase interpolation are needed to blend in-phase and quadrature-phase to yield a fine phase resolution. An similar approach was adopted by Altera Corp. in its EyeQ (Eye-Quality) monitors [60]. Table 3.1 lists some of recently reported on-chip EOMs.

Table 3.1: Eye-opening monitors

\begin{tabular}{|lllll|}
\hline Ref. & Tech. & EOM & Data rate & Year \\
\hline$[49]$ & SiGe & 1-dimen. & 10 Gbps & 2000 \\
{$[51]$} & $130 \mathrm{~nm}$ & 2-dimen. & 12.5 Gbps & 2005 \\
{$[61]$} & $180 \mathrm{~nm}$ & 1-dimen. & 10 Gbps & 2006 \\
{$[89]$} & $180 \mathrm{~nm}$ & 1-dimen. & 1.25 Gbps & 2007 \\
{$[62]$} & $180 \mathrm{BiCMOS}$ & 2-dimen. & 38-42 Gbps & 2008 \\
{$[54]$} & $180 \mathrm{~nm}$ & Jitter-based & 2 Gbps & 2008 \\
{$[90]$} & $180 \mathrm{~nm}$ & 2-dimen. & 5-10 Gbps & 2009 \\
\hline
\end{tabular}




\subsection{Proposed Two-Dimensional EOM}

In this section, we present a new two-dimensional hexagon on-chip EOM for Gbps serial links. The proposed hexagon EOM outperforms well known rectangular EOM and diamond EOM by accurately detecting the violation of the minimum eye opening.

\subsubsection{Drawbacks of Rectangular EOM}

Although the desired data symbols at the far-end channel are square waves, the loss of the channel results in the sinusoid-like waveform of the received symbol especially at a high symbol rate. Thus the rectangular EOM is week to depict the profile of the eye. To demonstrate this, consider Fig.3.3(a). The highlighted response cuts through the top-left corner of the eye mask at $t_{1}$. An error is detected at $t_{1}$.

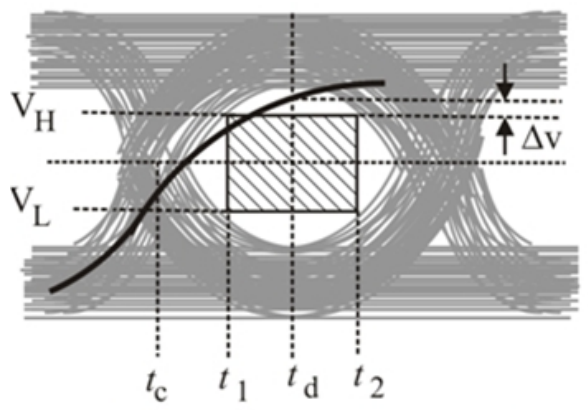

(a) Rectangular eye-opening monitor

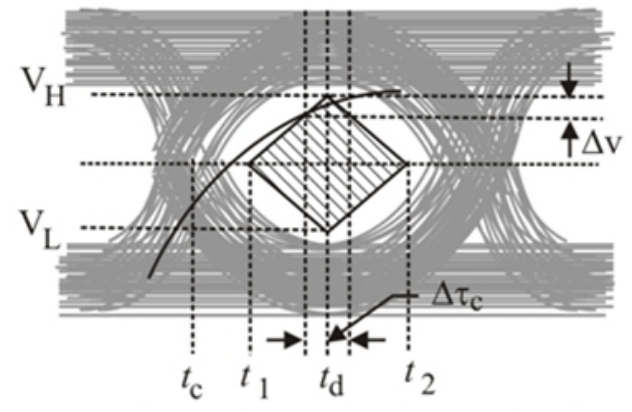

(b) Diamond eye-opening monitor

Figure 3.3: Rectangular and diamond EOMs.

Since the symbol is sampled at the center of the eye $\left(t=t_{d}\right)$ for data recovery and a voltage margin $\Delta v$ from the minimum eye-opening $V_{H}$ at $t=t_{d}$ exists, the received data will be safely recovered at $t=t_{d}$. This observation suggests that the highlighted trace should not be considered as an error. Another drawback of the rectangular EOM is its inability to impose a tight constraint on data jitter at the edge of the eye. The sinusoid-shaped response 
of the received data and the rectangular eye mask force $t_{1}$ to be far away from the eye edge $t_{c}$ for given $V_{H}$ and $V_{L}$ unless $V_{H}$ and $V_{L}$ are small.

\subsubsection{Diamond EOM}

The drawbacks of the aforementioned rectangular EOM can be eliminated by using the diamond-shaped EOM. It is seen in Fig.3.3(b) that the diamond EOM does not detect an error for the highlighted response that would otherwise flag an error if a rectangular EOM were used. The diamond EOM, however, does not take into the consideration of the effect of the jitter of the sampling clock at the center of the eye. The jitter of the sampling clock, denoted by $\Delta \tau_{c}$, gives rise to the uncertainty of the sampling time that is bounded by $t_{d}-\Delta \tau_{c} \leq t_{d} \leq t_{d}+\Delta \tau_{c}$. For the highlighted case shown in Fig.3.3(b), if the sampling point is at $t=t_{d}$ (no jitter), an error will be detected. However, if the sampling point is at $t=t_{d}+\Delta \tau_{d}$ due to the jitter of the sampling clock, no error will be detected.

\subsubsection{Hexagon EOM}

To take into account the effect of the jitter of the sampling clock and at the same time to tighten the budget of the data jitter at the edge of the eye, we propose the hexagon EOM shown in Fig.3.4. The vertical opening is defined by $V_{L}$ for the minimum and $V_{H}$ for the maximum while the horizontal opening is defined by $t_{1}$ to the left side and $t_{4}$ to the right side. The two design constraints of the hexagon EOM are: (i) $t_{2,3}$ should be chosen in such a way that $\left|t_{d}-t_{2,3}\right|>\Delta \tau_{c}$. This constraint ensures the jitter of the sampling clock will not affect the operation of the EOM. (ii) $t_{1}$ should be chosen such that $\left|t_{1}-t_{c}\right|>\Delta \tau_{d}$. This constraint ensures that the data jitter will not affect the operation of the EOM. A similar constraint should be imposed on $t_{4}$ as well.

The values of $t_{1}$ and $t_{2}$ for given $V_{H}$ and $V_{L}$ can be determined by assuming that the received 

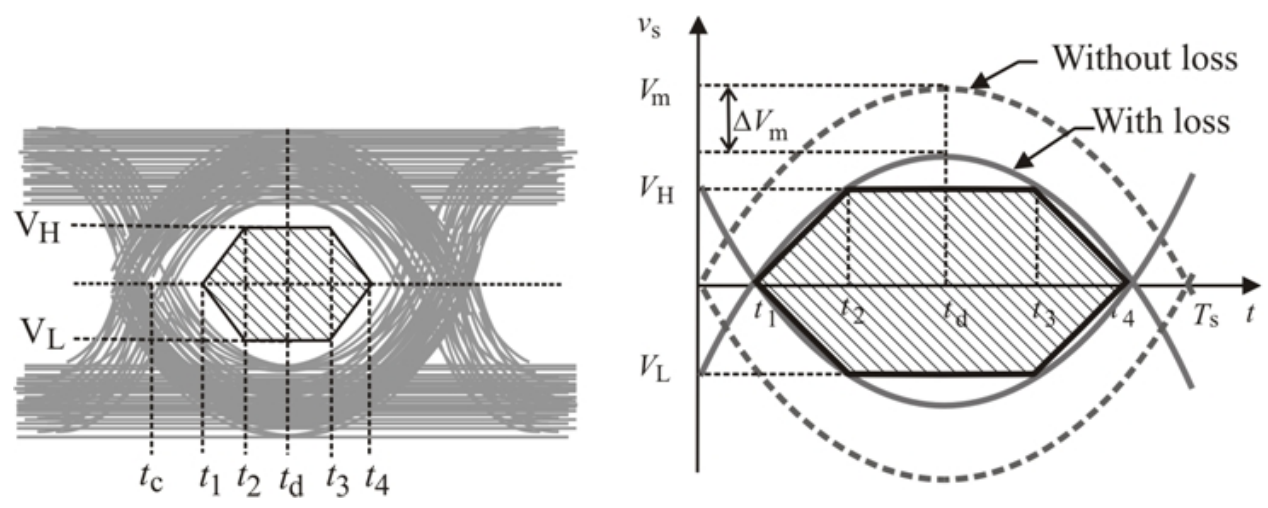

Figure 3.4: Hexagon EOM.

symbol without channel loss (the dotted sinusoid) is given : $v_{s}=V_{m} \sin \left(\omega_{s} t\right)$. The received symbol with channel loss (the solid sinusoid) is given by: $v_{s}=V_{m} \sin \left(\omega_{s} t\right)-\Delta V_{m}$. We thus have

$$
V_{m} \sin \left(\omega_{s} t_{1}\right)-\Delta V_{m}=0
$$

and

$$
V_{m} \sin \left(\omega_{s} t_{2}\right)-\Delta V_{m}=V_{H}
$$

from which we obtain

$$
t_{1}=\frac{1}{\omega_{s}} \sin ^{-1}\left(\frac{\Delta V_{m}}{V_{m}}\right)
$$

and

$$
t_{2}=\frac{1}{\omega_{s}} \sin ^{-1}\left(\frac{V_{H}+\Delta V_{m}}{V_{m}}\right) .
$$




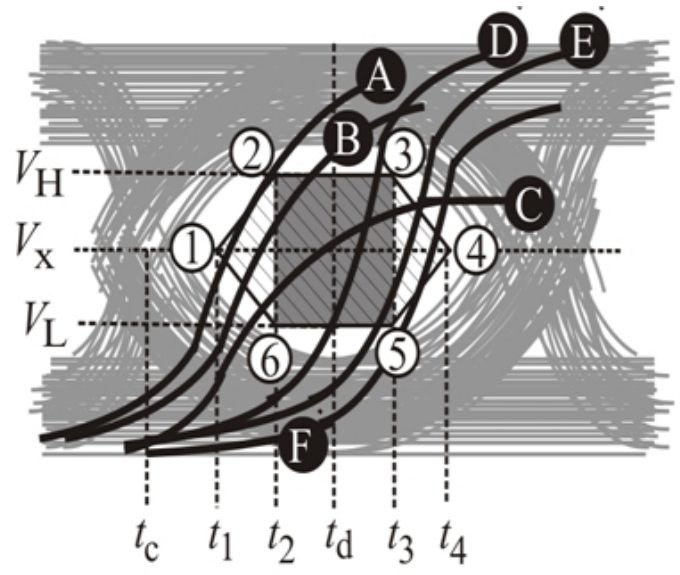

(a)

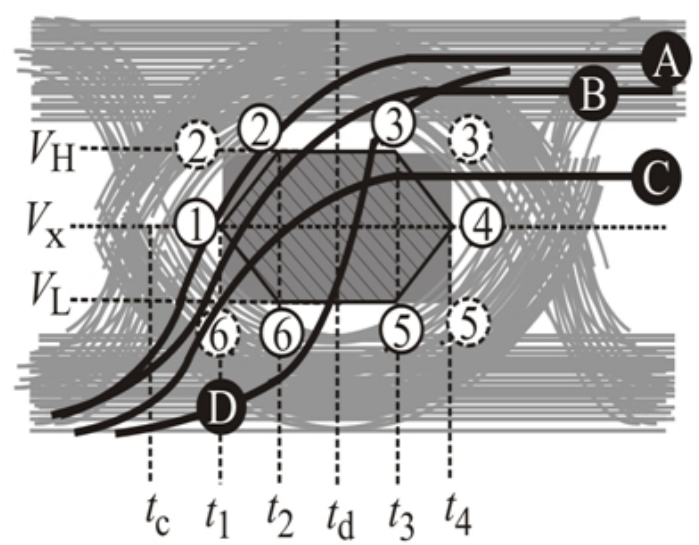

(b)

Figure 3.5: (a) Hexagon EOM that has the same vertical opening but different horizontal opening as that of rectangular EOM. (b) Hexagon EOM that has the same horizontal opening as that of rectangular EOM.

$t_{3}$ and $t_{4}$ can be determined by utilizing the symmetry of the eye with respect to the center of the eye. The improvement of the proposed hexagon EOM over the rectangular EOM can be demonstrated by considering a rectangular and a hexagon EOMs that have the same vertical opening $V_{L} \sim V_{H}$ :

1. Identical vertical opening (Fig.3.5(a)) : For the same vertical openings $V_{L} \sim V_{H}$, the upper bound of the data jitter is $\left|t_{2}-t_{c}\right|>\Delta \tau_{d}$ for the rectangular EOM and $\left|t_{1}-t_{c}\right|>$ $\Delta \tau_{d}$ for the hexagon EOM. With $t_{1}<t_{2}$, jitter constraint is tightened. Consider the highlighted traces in the figure: (i) Trace $\mathbf{A}$ : The rectangular EOM detects no error because $v_{s}\left(t_{2}\right)>V_{H}$ and $v_{s}\left(t_{3}\right)>V_{H}$ (The severity of error : low) while the hexagon EOM detects an error because $v_{s}\left(t_{1}\right)<V_{x}$ and $v_{s}\left(t_{2}\right)>V_{H}$ (The severity of error : low).

(ii) Trace B : The rectangular EOM detects an error at because $V_{L}<v_{s}\left(t_{2}\right)<V_{H}$ and $v_{s}\left(t_{3}\right)>V_{H}$ while the hexagon EOM detects two errors, one at $t_{1}$ because $v_{s}\left(t_{1}\right)<V_{x}$ and $v_{s}\left(t_{2}\right)>V_{H}$, and the other one at $t_{2}$ because $V_{L}<v_{s}\left(t_{2}\right)<V_{H}$. (iii) Trace $\mathbf{C}$ : The rectangular EOM detects two errors, one at $t_{2}$ because $V_{L}<v_{s}\left(t_{2}\right)<V_{H}$, and the other at $t_{3}$ because $V_{L}<v_{s}\left(t_{3}\right)<V_{H}$ while the hexagon EOM detects three errors, one at $t_{1}$ because $v_{s}\left(t_{1}\right)<V_{x}$ and $v_{s}\left(t_{2}\right)>V_{H}$, another at $t_{2}$ because $V_{L}<v_{s}\left(t_{2}\right)<V_{H}$, and 
the remaining one at $t_{3}$ because $V_{L}<v_{s}\left(t_{3}\right)<V_{H}$. (iv) Trace $\mathbf{D}$ : The rectangular EOM will detect an error because $V_{L}<v_{s}\left(t_{2}\right)$ and $v_{s}\left(t_{3}\right)>V_{H}$ while the hexagon EOM will also detect an error. (v) Trace $\mathbf{E}$ : It is the same as trace B. (vi) Trace F : It is the same as trace A.

2. Identical horizontal eye-opening (Fig.3.5(b)) : The hexagon and rectangular EOMs have the same horizontal opening defined by $t_{1}$ to the left and $t_{4}$ to the right. They also have the same vertical opening defined by $V_{H}$ the maximum and $V_{L}$ the minimum. In order to be consistent with Fig.3.5(a), the corners of the rectangular EOM are labeled 2,3,5 and 6. Consider the highlighted traces in the figure : (i) Trace A : The rectangular EOM detects an error at $t_{1}$ because $V_{L}<v_{s}\left(t_{1}\right)<V_{H}$ while the hexagon EOM detects no error at $t_{1}$ because $v_{s}\left(t_{1}\right)>V_{x}$ and $v_{s}\left(t_{2}\right)>V_{H}$. (ii) Trace B : The rectangular EOM detects an error at $t_{1}$ because $V_{L}<v_{s}\left(t_{1}\right)<V_{H}$ while the hexagon EOM detects two errors, one at $t_{1}$ because $v_{s}\left(t_{1}\right)<V_{x}$ and $v_{s}\left(t_{2}\right)>V_{L}$, and the other at $t_{2}$ because $v_{s}\left(t_{1}\right)<V_{x}$ and $V_{L}<v_{s}\left(t_{2}\right)<V_{H}$. (iii) Trace $\mathbf{C}$ : The rectangular EOM detects two errors, one at $t_{1}$ because $V_{L}<v_{s}\left(t_{1}\right)<V_{H}$, and the other $t_{4}$ because $V_{L}<v_{s}\left(t_{4}\right)<V_{H}$ while the hexagon EOM detects three errors, one at $t_{1}$, another at $t_{2}$, and the remaining one at $t_{3}$ because $v_{s}\left(t_{1}\right)<V_{x}$ and $V_{L}<v_{s}\left(t_{2}\right)<V_{H}$ and $V_{L}<v_{s}\left(t_{3}\right)<V_{H}$.

The preceding observations revels that the hexagon EOM provides a better error detection as compared with the rectangular EOM. Channel equalizers that utilizes the feedback provided by the hexagon EOM will outperform those with the rectangular EOM.

\subsubsection{Discussion}

Although in the preceding presentation of the proposed hexagon EOM, symmetrical data eyes were assumed, the proposed hexagon EOM can also handle asymmetrical data eyes. To illustrate, consider the minimum eye-opening mask with symmetrical vertical opening but 


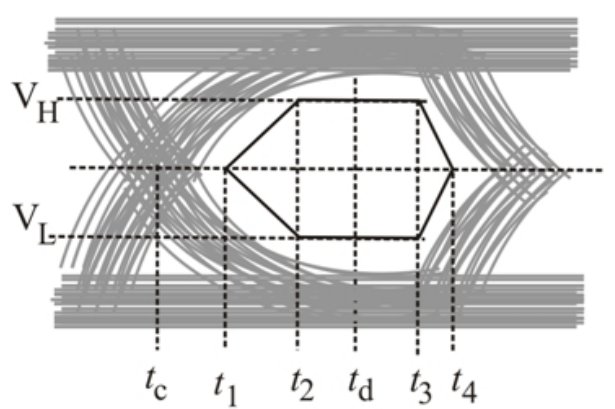

(a)

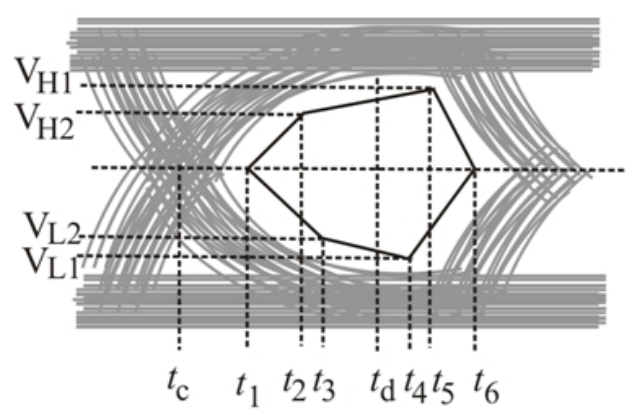

(c)

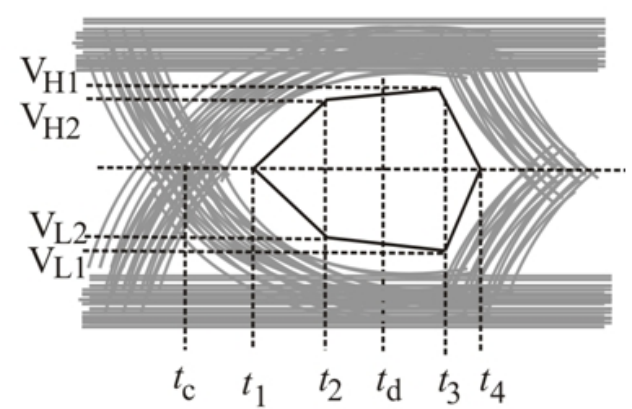

(b)

Figure 3.6: Asymmetrical hexagon EOMs.

asymmetrical horizontal opening, as shown in Fig.3.6(a). The sharp falling edges require that $t_{4}-t_{3}<t_{2}-t_{1}$ so that $t_{1}$ and $t_{4}$ can be placed close to the edges of the eye. Since $t_{1}, \ldots, t_{4}$ are obtained by delaying $t_{c}$ obtained from clock recovery, there is no additional cost to convert the symmetrical hexagon EOM to the asymmetrical hexagon EOM to handle asymmetrical data eyes. If the minimum vertical eye-opening mask is also not symmetrical, as shown in Fig.3.6(b), two additional threshold voltages and voltage comparators will be needed. In the extreme case where both the vertical and horizontal openings are not symmetrical, as shown in Fig.3.6(c), $t_{1}, \ldots, t_{6}$ need to be generated from $t_{c}$ and four voltage comparators are needed to detect any violation of the minimum eye-opening. The preceding observations show that the proposed hexagon EOM is robust in handling both symmetrical and asymmetrical data eyes.

Unlike serial links whose performance is mainly limited by ISI, the performance of parallel links are most constrained by data skew arising from the mismatch of parallel channels 
[1]. The close physical proximity of parallel channels and the finite channel length primarily constrained by the cost of routing a large number of parallel channels largely limit the data rate of parallel links per channel to significantly lower as compared with that of serial links. As a result, complex channel equalization schemes such as pre-emphasis and DFE are rarely needed in parallel links. If one chooses to use channel equalization in parallel links, the cost associated with it could be prohibitively high simply due to the large number of wire channels. However, if only one common DFE is used for all channels, the cost will be significantly reduced. In this case, the same eye-opening monitor is used for all channels. Since the characteristics of each channel might differ, $t_{1}, \ldots, t_{4}$ of each channel will also differ due to skew, one common eye-opening monitor with fixed $t_{1}, \ldots, t_{4}, V_{H}$, and $V_{L}$ might not be able to generate proper error signals subsequently providing optimal DFE equalization. 


\subsection{Implementation}

To verify the performance of the proposed EOM, a data link shown in Fig.3.7, is designed in IBM $130 \mathrm{~nm}$ 1.2V CMOS technology.

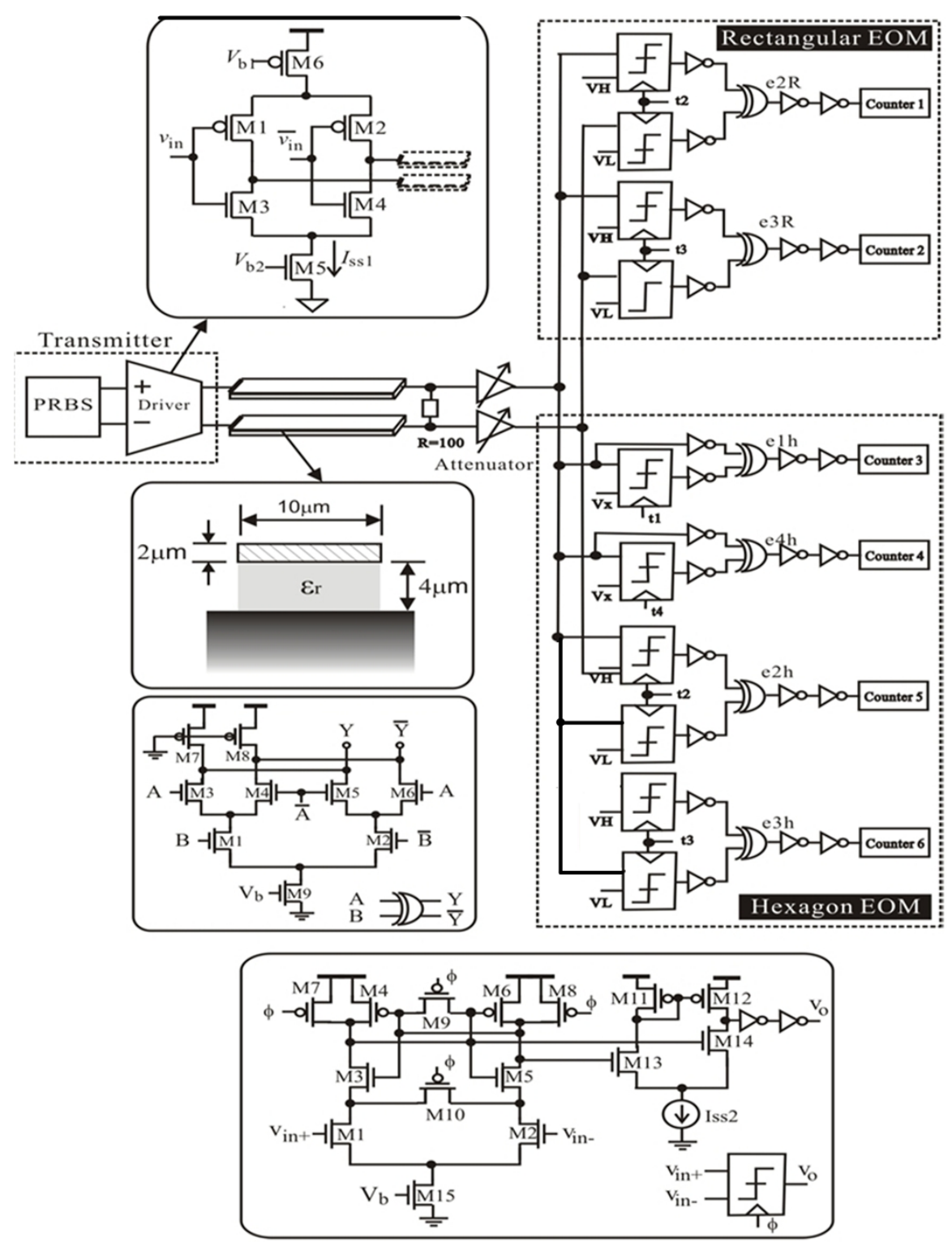

Figure 3.7: Serial link with a hexagon EOM. For the purpose of comparison, a rectangular EOM in parallel with the hexagon EOM is also employed. 
The transmitters consist of a pseudo-random generator with BER of $2^{31}-1$ and a differential current-mode driver that conveys a $2 \mathrm{~mA}$ current to the channel. Note that current-mode signaling is widely preferred in Gbps serial links due to their intrinsic advantages such as a low voltage swing subsequently high data rates, low switching noise, and well-defined output impedance allowing a better impedance matching possible. In contrast, non-linear drivers, such as static inverter-based drivers, have a significant effect on the signal integrity. These drivers not only produce a large voltage swing, which in turn limits data rates and consumes more power, they also generate large switching noise and substrate noise. The varying output impedance of these drivers also introduces a large impedance mismatch subsequently a strong inter-symbol interference. The channel is a 1-mm transmission line with its physical dimensions shown in the figure. The channel is terminated with a $100 \Omega$ resistor. The resistor also performs current-to-voltage conversion. Two voltage attenuators are used to adjust the level of the received signal so as to emulate the attenuation of the channel subsequently test the proposed EOM. A hexagon EOM and a rectangular EOM are connected in parallel, allowing them to process the same symbol simultaneously so that their performance can be compared. The minimum vertical opening of the eye is set to $V_{H}=0.8 \mathrm{~V}$ the maximum and $V_{L}=0.6 \mathrm{~V}$ the minimum for both EOMs. The minimum horizontal opening of the rectangular EOM is set by $t_{1}=0.2 \mathrm{~ns}$ to the left and $t_{2}=0.8 \mathrm{~ns}$ to the right. The minimum opening of the hexagon eye-opening is set to $t_{1}=0.2 \mathrm{~ns}$ to the left, $t_{2}=0.4 \mathrm{~ns}, t_{3}=0.6$ $\mathrm{ns}$, and $t_{4}=0.8 \mathrm{~ns}$ to the right. The data rate used for simulation is 1 Gbps (symbol time $\left.T_{s}=1 \mathrm{~ns}\right)$. The link is analyzed using Spectre from Cadence Design Systems with BSIM 4 device models.

Fig.3.8 shows the simulation results of the rectangular EOM with $V_{H}=0.85 \mathrm{~V}$ and $V_{L}=0.65 \mathrm{~V}$. The voltage of the received symbol exceeds $V_{H}$ and $V_{L}$, i.e., $v_{i n, \max }>V_{H}$ and $v_{\text {in,min }}<V_{L}$. No error is detected at sampling times $S t_{2}$ and $S t_{3}$.

Fig.3.9 shows the simulation results of the rectangular EOM with $V_{H}=0.85 \mathrm{~V}$ and $V_{L}=0.65 \mathrm{~V}$. Using attenuators to attenuate the received symbol such as $v_{i n, \max }<V_{H}$ while $v_{\text {in,min }}<V_{L}$. Hence, errors are detected at sampling times $S t_{2}$ and $S t_{3}$. 


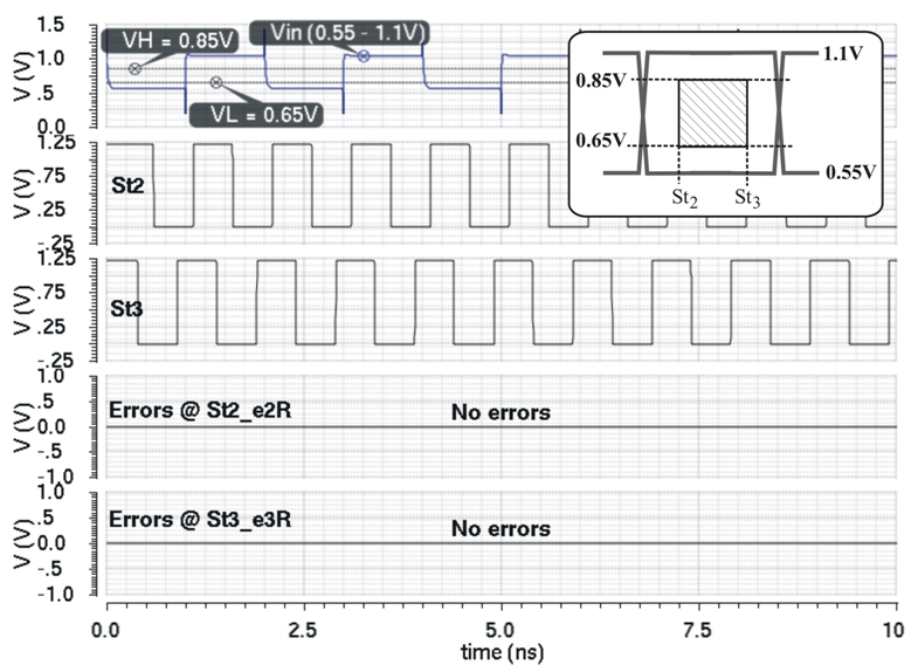

Figure 3.8: Simulated output of the error generators of rectangular EOM with $v_{\text {in,max }}>V_{H}$ and $v_{\text {in, } \min }<V_{L}$.

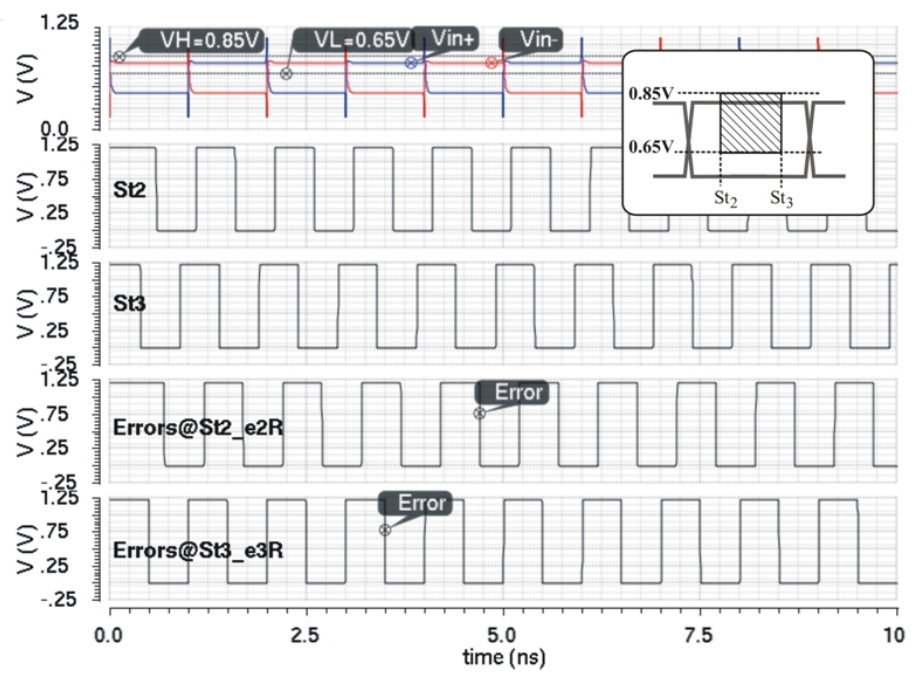

Figure 3.9: Simulated output of the error generators of rectangular EOM with $v_{i n, \max }<V_{H}$ and $v_{\text {in, } \min }<V_{L}$.

Fig.3.10 shows the simulation results of the rectangular EOM with $V_{H}=0.85 \mathrm{~V}$ and $V_{L}=$ $0.45 \mathrm{~V}$. The voltage of the received symbol satisfies $v_{i n, \max }>V_{H}$ but $v_{i n, \min }>V_{L}$. As a result, errors are detected at sampling instants $S t_{2}$ and $S t_{3}$ due to $v_{i n, \min }>V_{L}$ and $v_{\text {in,max }}>V_{H}$. Fig.3.11 shows the simulation results of the hexagon EOM with $V_{H}=0.85 \mathrm{~V}$ and $V_{L}=0.65 \mathrm{~V}$. 
The voltage of the received symbol exceeds $V_{H}$ and $V_{L}$, i.e., $v_{i n, \max }>V_{H}$ and $v_{i n, \min }<V_{L}$. As a result, no error is detected at sampling instants $S t_{2}$ and $S t_{3}$.

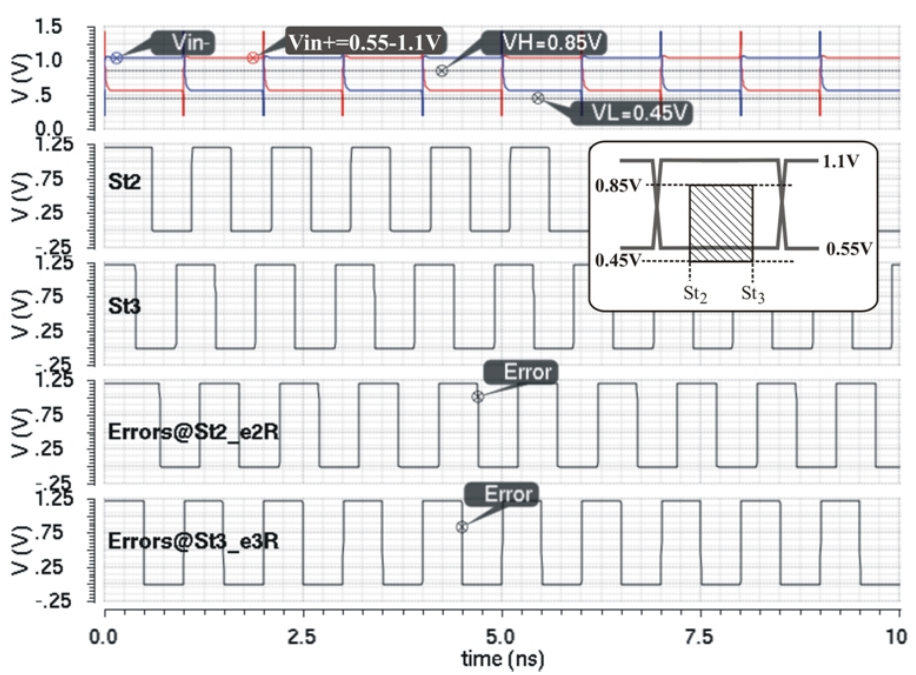

Figure 3.10: Simulated output of the error generators of rectangular EOM with $v_{i n, \max }>V_{H}$ and $v_{\text {in, } \min }>V_{L}$.

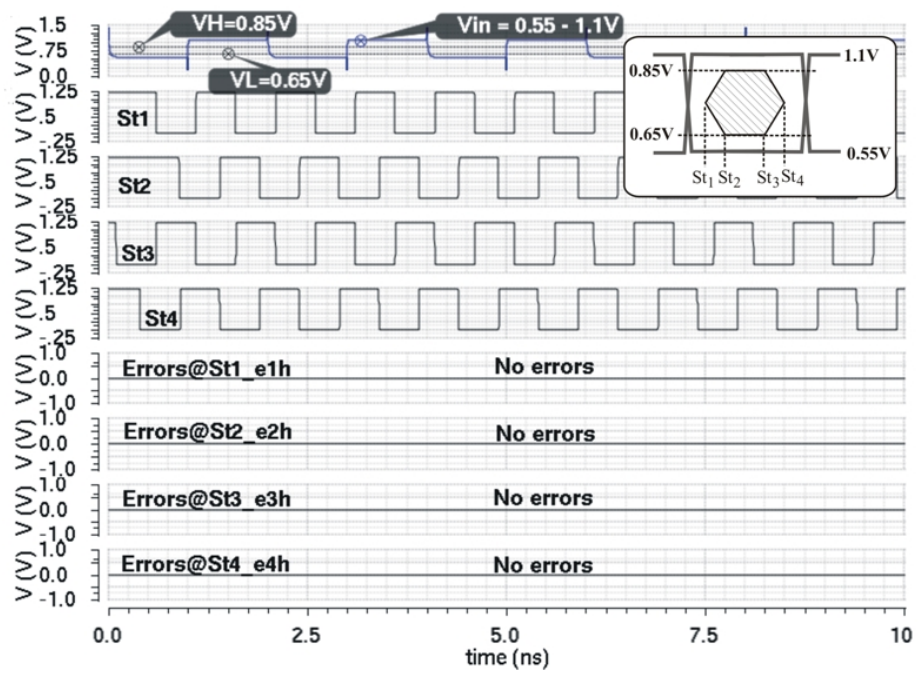

Figure 3.11: Simulated output of the error generators of hexagon EOM with $v_{i n, \min }<V_{L}$ and $v_{\text {in, } \max }>V_{H}$.

Fig.3.12 shows the simulation results of the rectangular EOM with $V_{H}=0.85 \mathrm{~V}$ and $V_{L}=$ $0.45 \mathrm{~V}$. The voltage of the received symbol is attenuated using the attenuators such as $v_{i n, \max }<V_{H}$ but $v_{i n, \min }<V_{L}$. As a result, errors are detected at sampling instants $S t_{2}$ 
and $S t_{3}$ due to $v_{i n, \max }<V_{H}$.

Fig.3.13 shows the simulation results of the hexagon EOM with $V_{H}=0.85 \mathrm{~V}$ and $V_{L}=0.45 \mathrm{~V}$.

The voltage of the received symbol satisfies $v_{i n, \max }>V_{H}$ but $v_{i n, \min }>V_{L}$. As a result, errors are detected at sampling instants $S t_{2}$ and $S t_{3}$ due to $v_{i n, \min }>V_{L}$ and $v_{i n, \max }>V_{H}$.

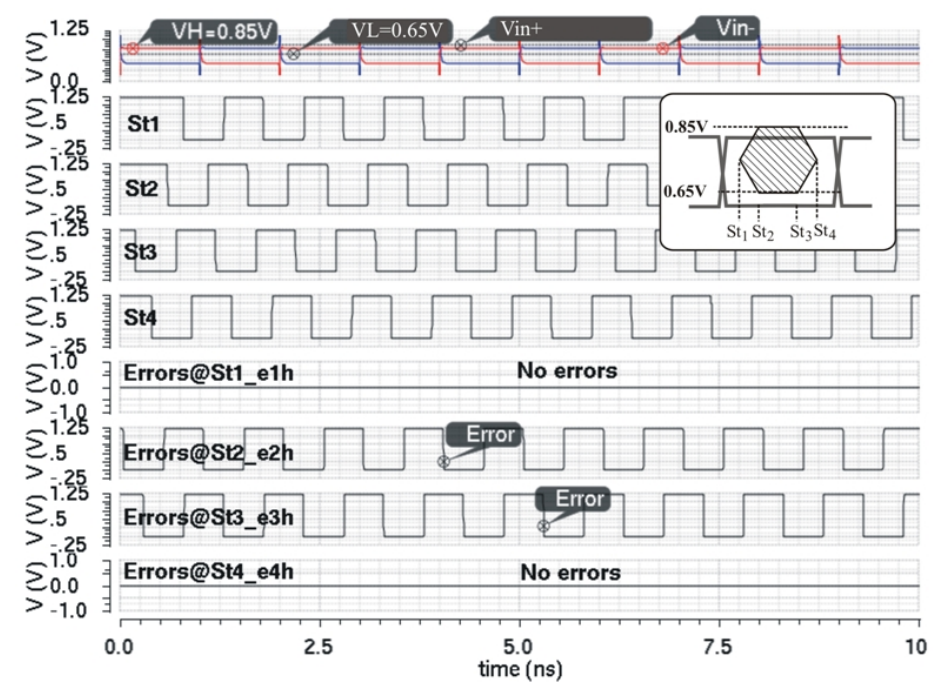

Figure 3.12: Simulated output of the error generators of hexagon EOM with $v_{i n, \max }<V_{H}$ and $v_{i n, \min }<V_{L}$.

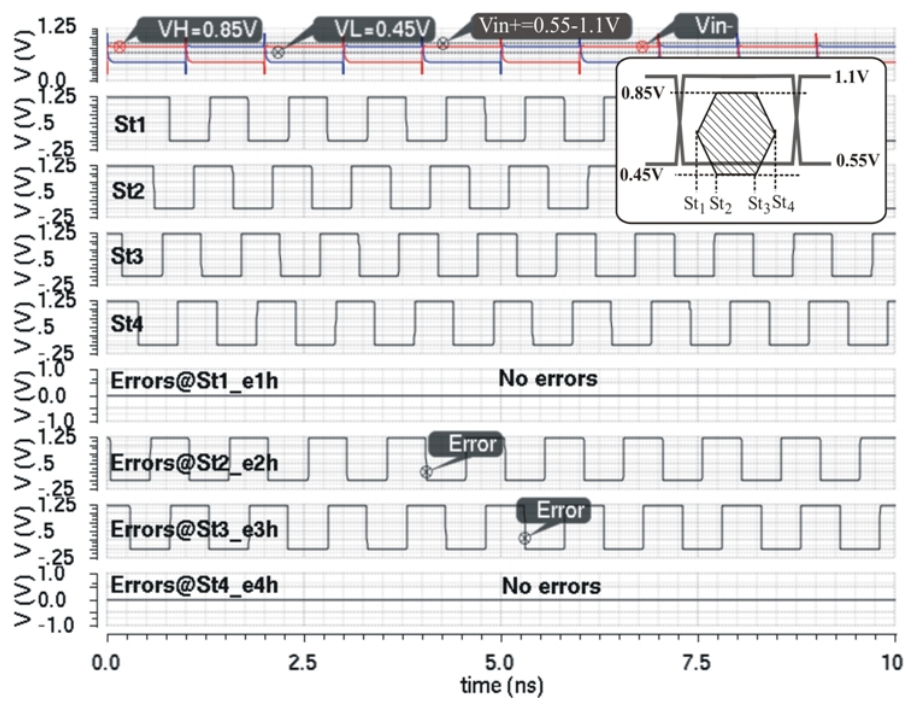

Figure 3.13: Simulated output of the error generators of hexagon EOM with $v_{i n, \min }>V_{L}$ and $v_{i n, \max }>V_{H}$. 
Fig.3.14 plots the waveform of the received data symbols at the input of the comparators and that of the output of the error generators of the hexagon and rectangular EOMs. No data jitter is included in simulation. As can be seen that the voltage swing of the received data symbol exceeds $V_{H}=0.8 \mathrm{~V}$ and $V_{L}=0.6 \mathrm{~V}$, no error is generated by the rectangular EOM and by the hexagon EOM. These results agree well with the analytical results presented earlier. Note that the sparks at the output of the error generators are due to the use of ideal sampling clocks in our simulation and will disappear once real sampling clocks are used.

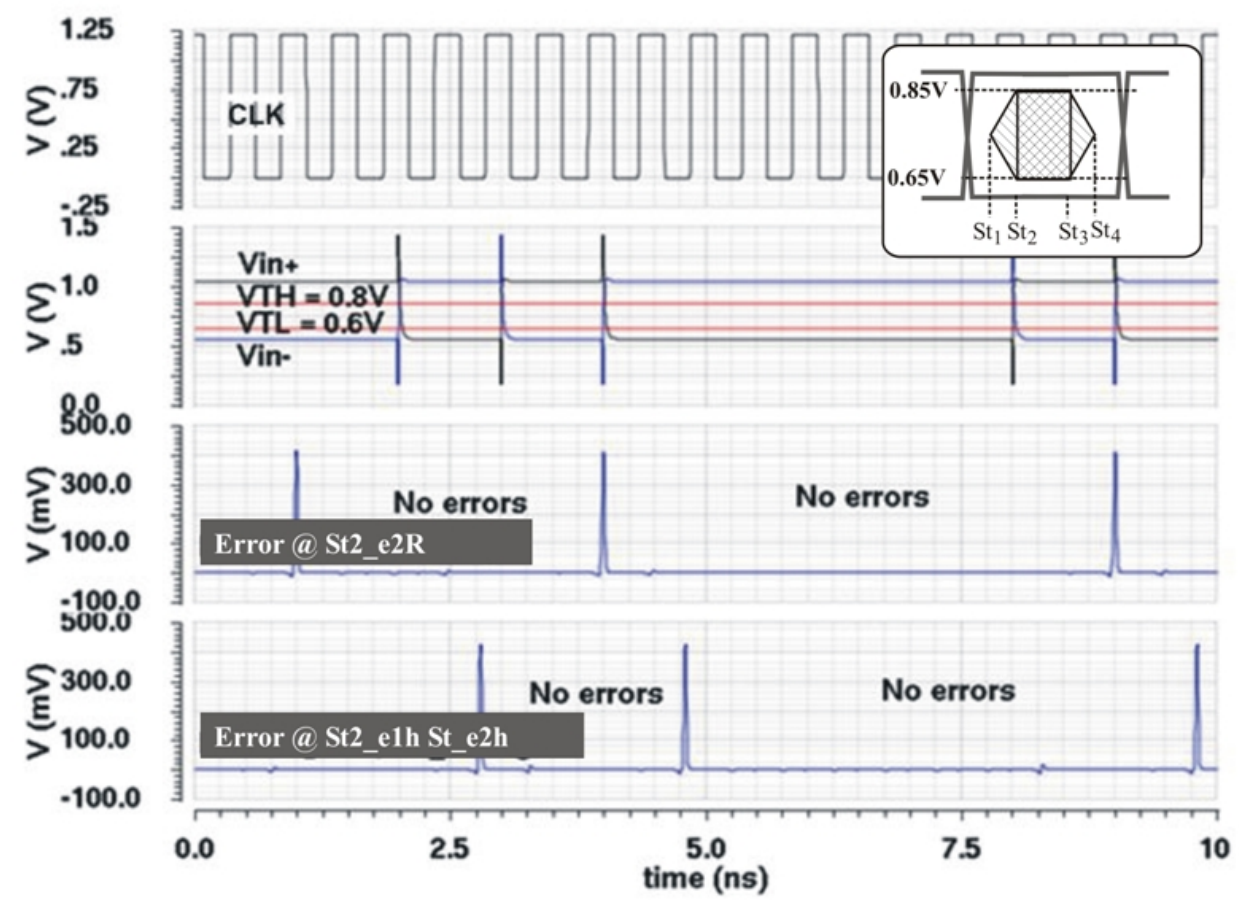

Figure 3.14: Simulated output of the error generators of rectangular EOM and hexagon EOM with a 1-mm channel, $v_{i n, \min }<V_{L}$, and $v_{i n, \max }>V_{H}$.

To investigate the effect of channel loss on both EOMs, the length of the channel is increased to $40 \mathrm{~mm}$ and the response of the received data symbol, together with the output of the error generators of the hexagon and rectangular eye-opening monitors are shown in Fig.3.15. It is seen that an error is detected by the rectangular EOM while no error is detected by the hexagon EOM. 


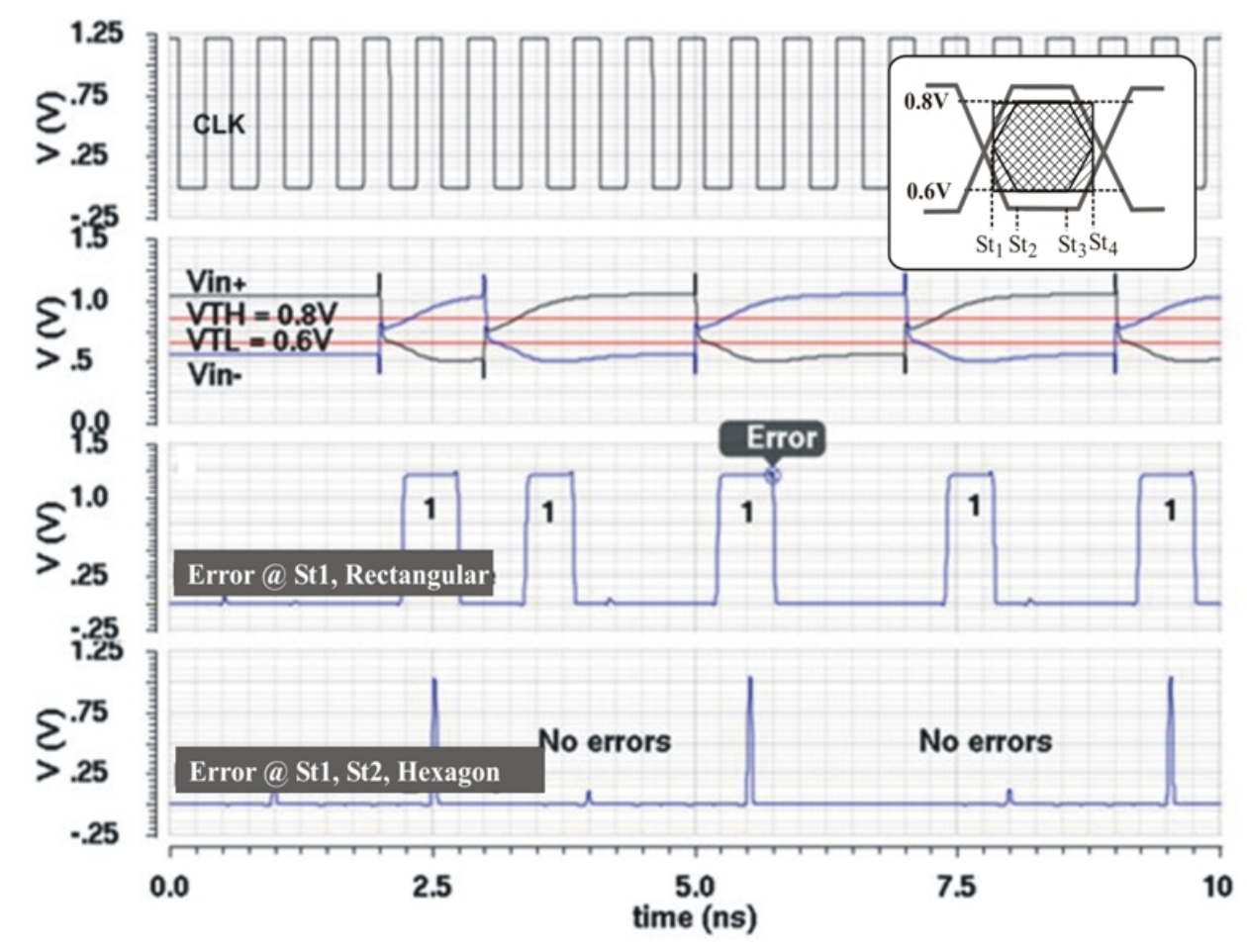

Figure 3.15: Simulated output of the error generators of rectangular EOM and hexagon EOM with a 40-mm channel.

To further investigate the effect of channel loss on both EOMs, the length of the channel is increased to $200 \mathrm{~mm}$ and the response of the received data symbol, together with the output of the error generators of the hexagon and rectangular eye-opening monitors are shown in Fig.3.16. The attenuation of the channel causes the rectangular EOM to detect a violation of the minimum eye-opening at corner 2 of the rectangular EOM (severity of error : low) while the hexagon eye-opening monitor detects two violation of the minimum eye-opening at corners 1 and 2 (severity of error : moderate). 


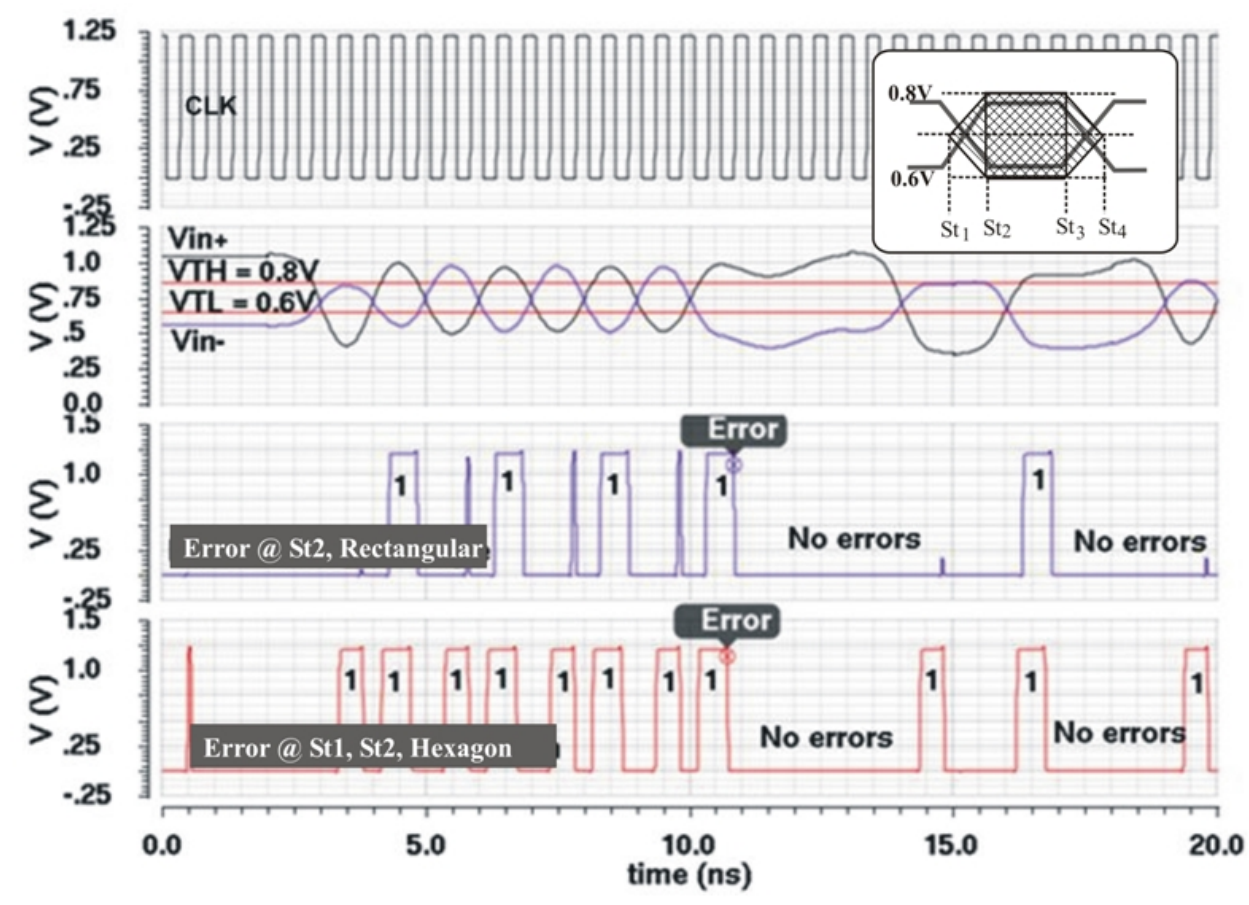

Figure 3.16: Output of error generator of hexagon EOM.

\subsection{Chapter Summary}

A comprehensive review of the state-of-the-art of on-chip EOMs was provided and their pros and cons were studied. A new two-dimensional hexagon EOM that outperforms the widely used rectangular two-dimensional EOM was introduced. The implementation details of the proposed EOM were provided. The effectiveness of the proposed two-dimensional EOM was assessed by embedding it in a serial link implemented in an IBM $130 \mathrm{~nm} 1.2 \mathrm{~V}$ CMOS technology. For the purpose of comparison, a rectangular two-dimensional EOM was also included in the data link. The simulation results of the data link demonstrate that the proposed two-dimensional EOM outperforms the rectangular two-dimensional EOM by providing a tightened control of data jitter at the edge of data eyes and by eliminating unnecessary errors flagged from the rectangular EOM. 


\section{Chapter 4}

\section{2-Dimensional Half-Hexagon Eye- Opening Monitor}

This chapter proposes a power-efficient half hexagon EOM for Gbps serial links. The proposed EOM consumes less power and silicon as compared with 2-dimensional hexagon EOM presented in the previous chapter. The remainder of the chapter is organized as the followings: Section 4.1 presents the proposed half hexagon EOM. The implementation of the EOM is detailed in Section 4.2. The simulation results of the proposed EOM are presented in Section 4.3. The chapter is summarized in Section 4.4.

\subsection{Half Hexagon Eye-Opening Monitor}

It has been observed that at high data rates, the data eye is approximately symmetrical with respect to the center of the eye, the detection of whether there exists a violation of the minimum eye-opening mask or not is therefore only needed to perform on half of the eye, as illustrated graphically in Fig.4.1.

It is seen that the violation of the minimum eye-opening mask at $t_{3}$ can be determined by that at $t_{2}$. This observation reveals that the violation of the minimum eye-opening mask can be detected using only half of the hexagon mask, more specifically, the left half of the hexagon mask. We use Fig.4.2 to further demonstrate this. Consider the following cases : (i) Trace A : The full hexagon EOM, Fig.4.2(right), detects no error because $v\left(t_{j}\right)>V_{H}$ 
for $j=2,3$ and $v\left(t_{j}\right)>V_{x}$ for $j=1,4$. The half hexagon rectangular EOM, Fig.4.2(left), also detects no error.
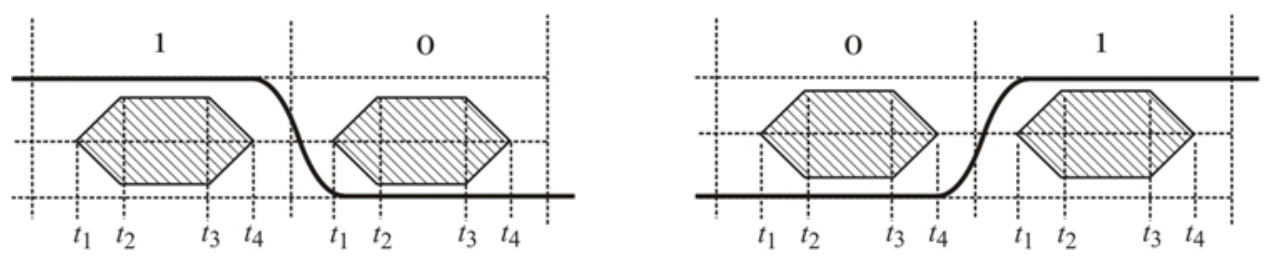

(a) No violation of the minimum eye-opening mask
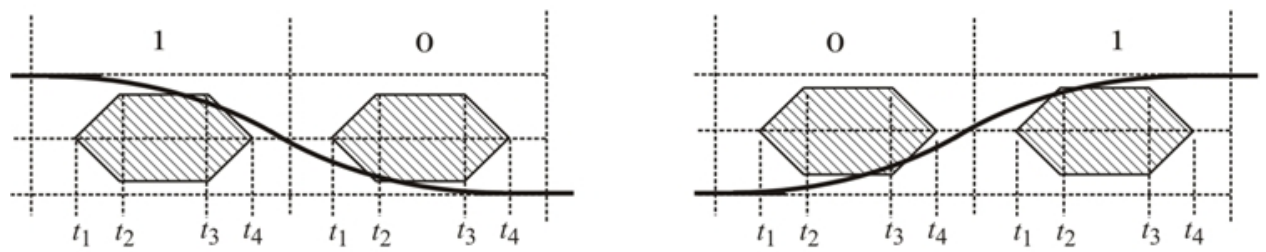

(b) Violation of the minimum eye-opening mask

Figure 4.1: Detection of violation of the minimum eye-opening using half hexagon mask.

(ii) Trace B : The full hexagon EOM detects errors at $t_{1}$ and $t_{2}$ and no error at $t_{3}$ and $t_{4}$. The half hexagon EOM also detects errors $t_{1}$ and $t_{2}$.
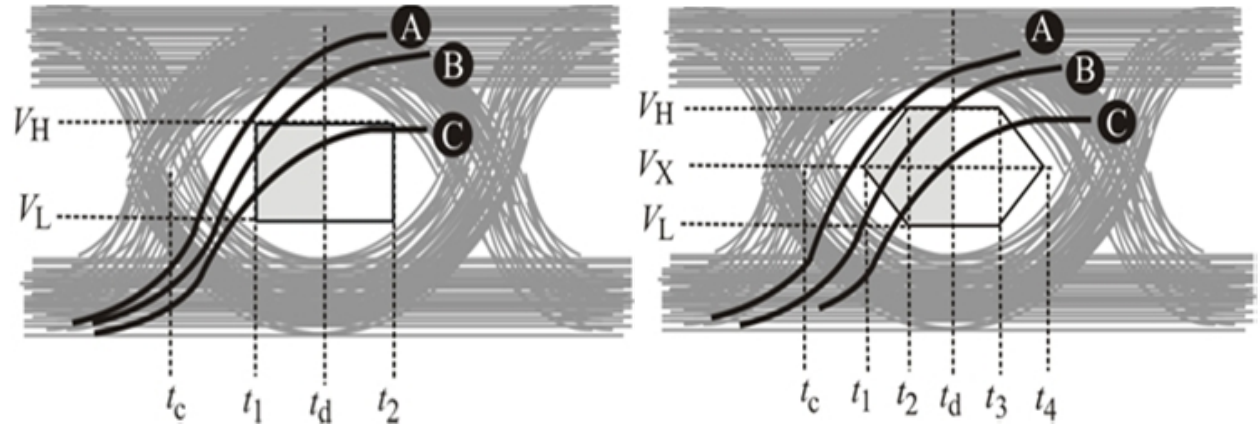

Figure 4.2: Left : Full and half rectangular Eye-Opening patterns. Right : Full and half hexagon Eye-Opening patterns 
(iii) Trace $\mathbf{C}$ : The full hexagon EOM detects errors at $t_{1}, t_{2}$, and $t_{3}$ but no error at $t_{4}$. The half hexagon EOM detects errors at $t_{1}$ and $t_{2}$. The symmetry of the data eye indicates that a violation of the minimum eye-opening mask at $t_{3}$ also exists. The preceding observations reveal that the full and half hexagon EOMs will flag the same error. Since four voltage comparators and four delay blocks are needed for the full-hexagon EOM and only two voltage comparators and two delay blocks are needed for the half-hexagon EOM, the silicon and power consumption of the half-hexagon EOM is only half that of the full-hexagon EOM. A similar analysis can be conducted for the rectangular EOM shown in Fig.4.2(left) as well.

\subsection{Implementation}

The configuration of full and half hexagon EOMs are shown in Fig.4.3. The output of the

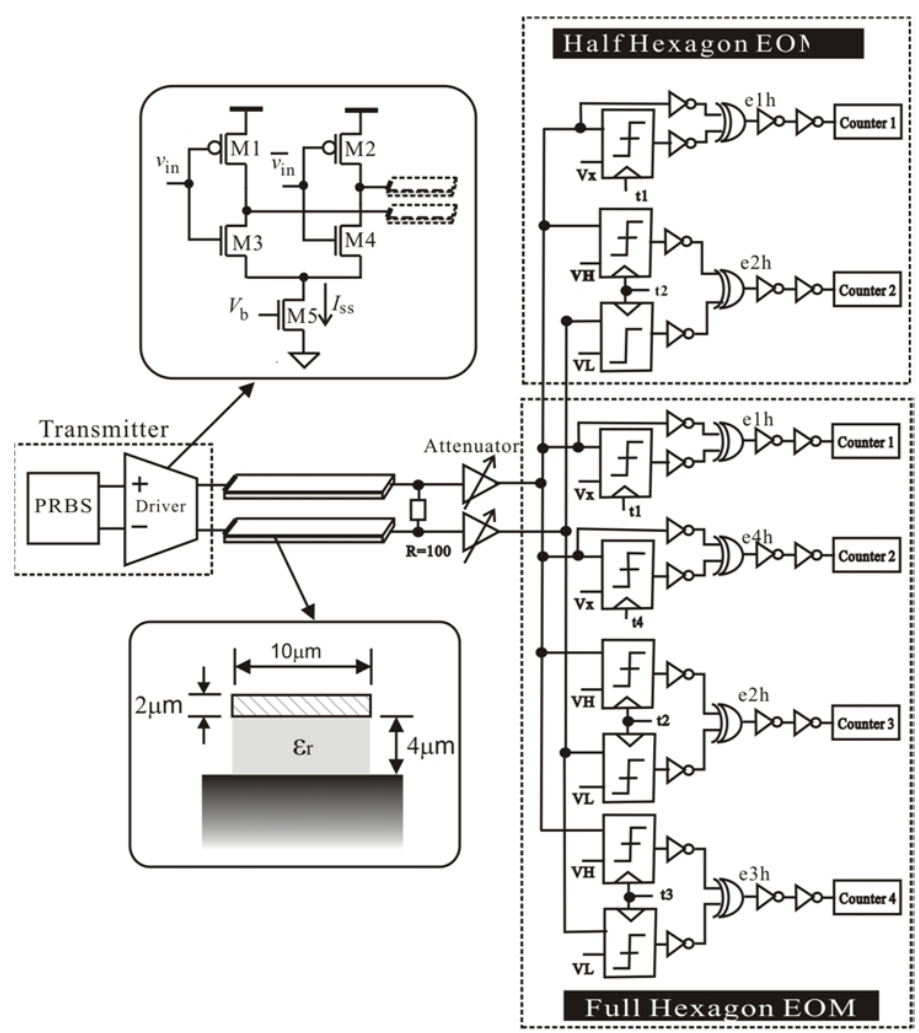

Figure 4.3: Serial link with half and full hexagon EOMs. Circuit parameters : $W_{1,2}=6.5 \mu \mathrm{m}$, $W_{3,4}=4 \mu \mathrm{m}, W_{5}=50 \mu \mathrm{m}$. $L=0.13 \mu \mathrm{m}$ for all transistors. $V_{b}=0.7 \mathrm{~V}$ with tail current $I_{s s}=2 \mathrm{~mA}$. 
comparators is fed to logic blocks to generateerror signals once a violation of the minimum eye-opening mask is detected. The error signals are fed to the counters to record the number of violation. The schematic of the clocked comparator is shown in Fig.4.4.

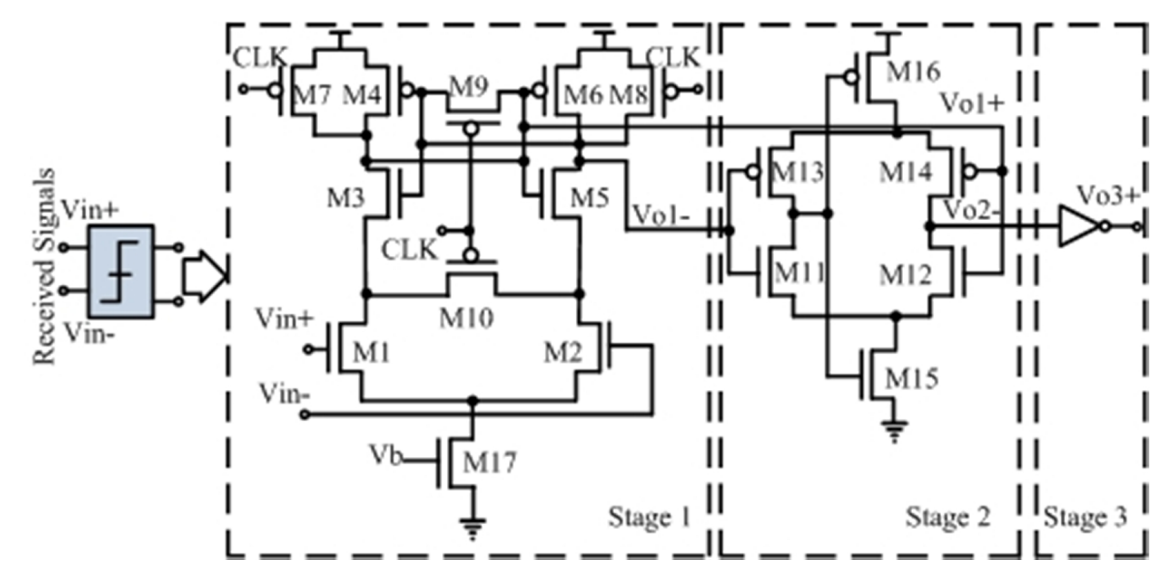

Figure 4.4: Clocked comparator. Circuit parameters : Circuit parameters : $W_{1,2}=4.5 \mu \mathrm{m}$, $W_{3,5}=4 \mu \mathrm{m}, W_{4,6,7,8}=8 \mu \mathrm{m}, W_{9,10}=1 \mu \mathrm{m}, W_{11,12}=1 \mu \mathrm{m}, W_{13,14}=2 \mu \mathrm{m}$, and $W_{15,16,17}=60 \mu \mathrm{m}$. $L=0.13 \mu \mathrm{m}$ for all transistors. $I_{s s}=2 \mu \mathrm{A}, V_{b}=0.8 \mathrm{~V}$.

The design philosophy of the multi-stage comparator proposed by Fayerd and Ismail in [63] was followed in this work to minimize the effect of duty-cycle distortion [64] and kick-back [65]. The first stage is a clocked differential amplifier with a cross-coupled inverter pair load to amplify the differential symbol signal. The positive feedback formed by the cross-coupled inverter pair ensures the rapid transition of the output of the comparator. The output of the first stage is fed to a self-biased differential amplifier that performs further amplification and differential to single-ended conversion. The 3rd and 4th stages are simply static inverters. The deployment of cross-coupled inverter pair in stage 1 ensures that the output of the stage 1 will switch at the same time dispite of the mismatches between M1 and M2, and that between M3/M4 inverter pair and M5/M6 inverter pair. Similarly, the self-biased differential pair in stage 2 will also minimize duty-cycle distortion. Note that if the stage 1 was implemented using a differential pair, the mismatches between the input transistors of 
the differential pair would give rise to differential triggering voltages of the comparator. If we assume that the triggering voltage variation is $\Delta v$ and further assume that the sampling clock is jitter-free, $\Delta v$ will directly affect the detection of the violation of the minimum eye-opening mask, as illustrated graphically in Fig.4.5.

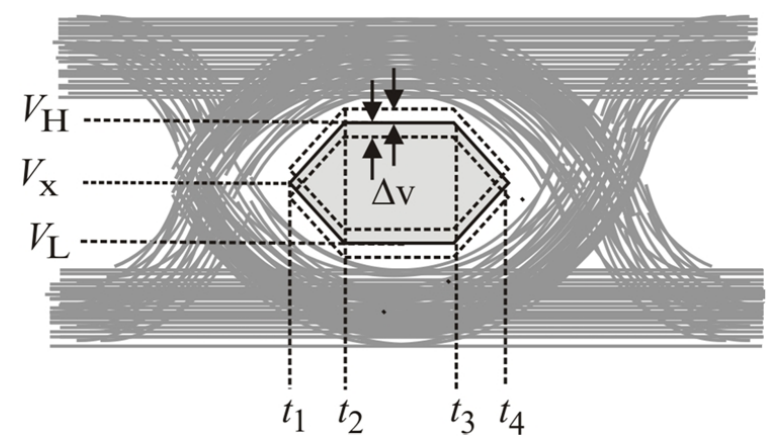

Figure 4.5: Effect of duty-cycle distortion on hexagon EOM.

The schematic of the current-mode differential XOR2 gate is shown in Fig.4.6.

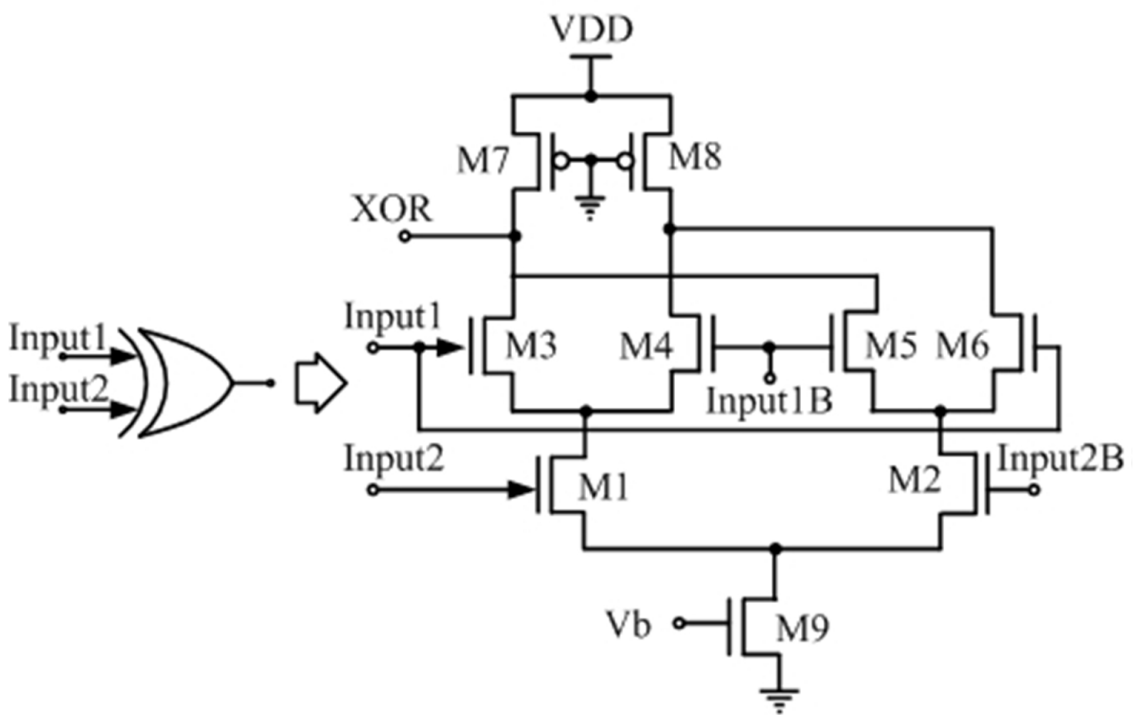

Figure 4.6: Schematic of XOR2. Circuit parameters: $W_{1,2}=3 \mu m, W_{7,8}=1.5 \mu m$, and $W_{9}=$ $50 \mu m$. $L=0.13 \mu m$ for all transistors, $V_{b}=0.8 \mathrm{~V}$. 
Current-mode logic is known for its superior speed and low switching noise generation. To improve the speed of the circuit, the load resistors are replaced with two PMOS transistors $M_{7,8}$ working in the triode region. For the purpose of comparison, a full rectangular EOM and a half rectangular EOM shown in Fig.4.7 were also designed, and their performance is compared with that of hexagon EOM.

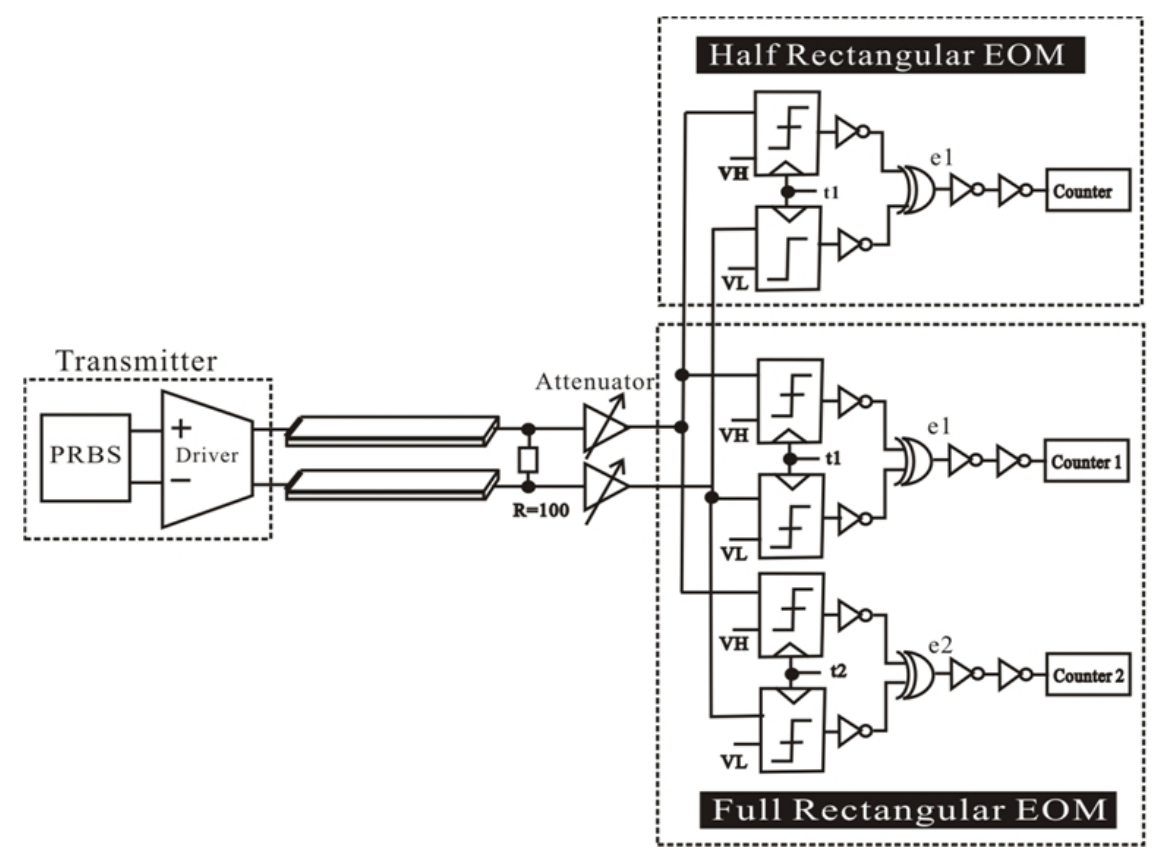

Figure 4.7: Serial link with half and full rectangular EOMs. The same circuit and channel parameters as those in Fig.4.3 are used.

\subsection{Simulation Results}

To verify the performance of the proposed half hexagon EOM, the data link shown in Fig.4.3

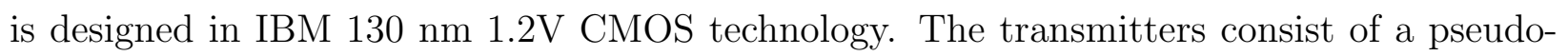
random generator with BER of $2^{31}-1$ and a differential current-mode driver that conveys a $2 \mathrm{~mA}$ current to the channel. The channel is a 1-mm transmission line terminated with a $100 \Omega$ resistor. Two voltage attenuators are used to adjust the level of the received signal so 
as to emulate the loss of the channel. For the purpose of comparison, the data link with fullrectangular and half-rectangular EOMs and the same transmitter and channel (Fig.4.7) is also designed. The minimum vertical opening of the eye is set to $V_{H}=0.85 \mathrm{~V}$ and $V_{L}=0.65$ $\mathrm{V}$. The minimum horizontal opening of the rectangular EOM is set by $t_{1}=0.2 \mathrm{~ns}$ to the left and $t_{2}=0.8 \mathrm{~ns}$ to the right while that of the hexagon EOM is set to $t_{1}=0.2$ ns to the left, $t_{2}=0.4 \mathrm{~ns}, t_{3}=0.6 \mathrm{~ns}$, and $t_{4}=0.8 \mathrm{~ns}$ to the right. The data rate is 1 Gbps. The links are analyzed using Spectre from Cadence Design Systems with BSIM4 device models. Fig.4.8 shows the simulation results of the full hexagon EOM with the voltage swing of the received symbol exceeding $V_{H}$ and $V_{L}$. As a result, no error is detected at sampling instants $S t_{2}$ and $S t_{3}$.

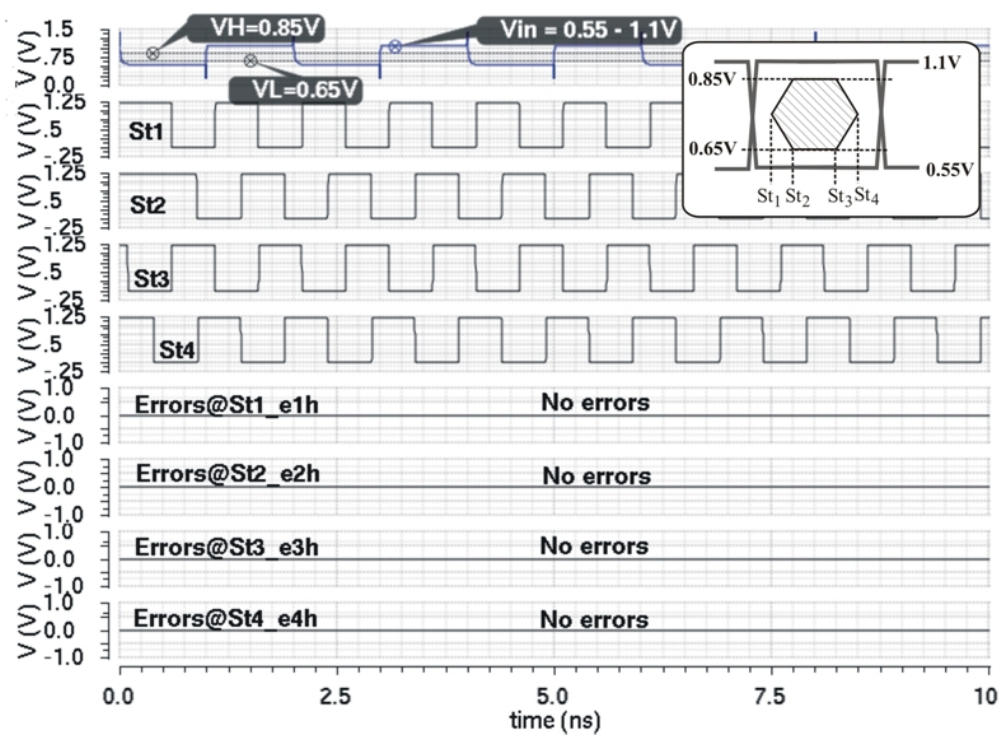

Figure 4.8: Simulated output of full hexagon EOM with $v_{\text {in, } \min }<V_{L}$ and $v_{\text {in, } \max }>V_{H}$. No error is detected at $S t_{2}$ and $S t_{3}$. Top plot : Received data. 2nd-5th plots : Sampling clocks $S t_{2} \sim S t_{4}$. 6th-9th plots : Error outputs $e 1 h \sim e 4 h$.

Fig.4.9 shows the simulation results of the half hexagon EOM with the same input and the same $V_{H}$ and $V_{H}$. No error is detected at sampling instants $S_{t 2}$ and $S_{t 3}$. 


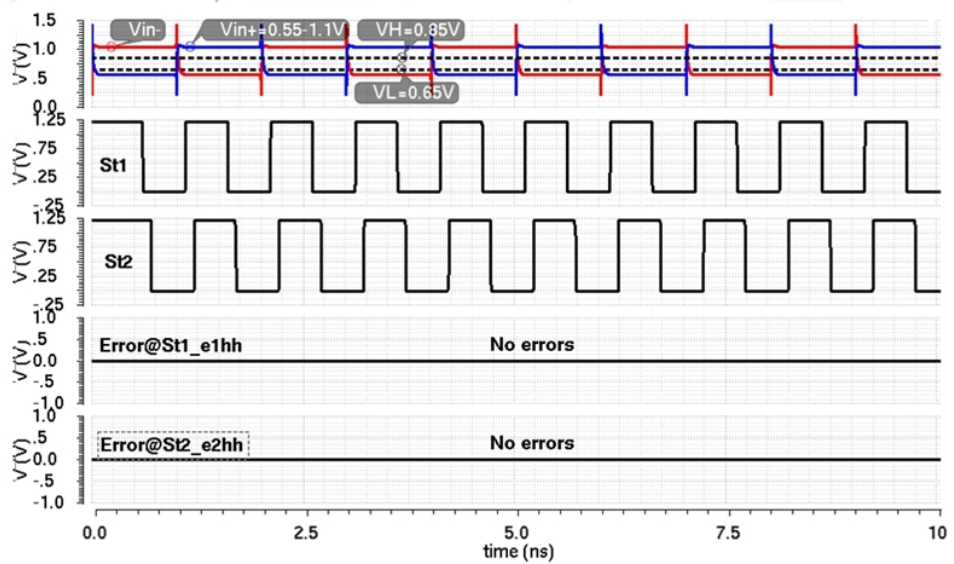

Figure 4.9: Simulated output of half hexagon EOM with $v_{i n, \min }<V_{L}$ and $v_{i n, \max }>V_{H}$. No error is detected at $S t_{2}$ and $S t_{3}$. Top plot : Received data. 2nd-3rd plots : Sampling clocks $S t_{2} \sim S t_{2}$. 4th-5th plots : Error outputs $e 1 h \sim e 2 h$.

Figs.4.10 and 4.11 show the simulation results of the full and half hexagon EOMs, respectively with $v_{i n, \max }<V_{H}$ and $v_{i n, \min }<V_{L}$. Errors are detected at $S t_{2}$ and $S t_{3}$. It is seen that both EOMs provide the same error detection.

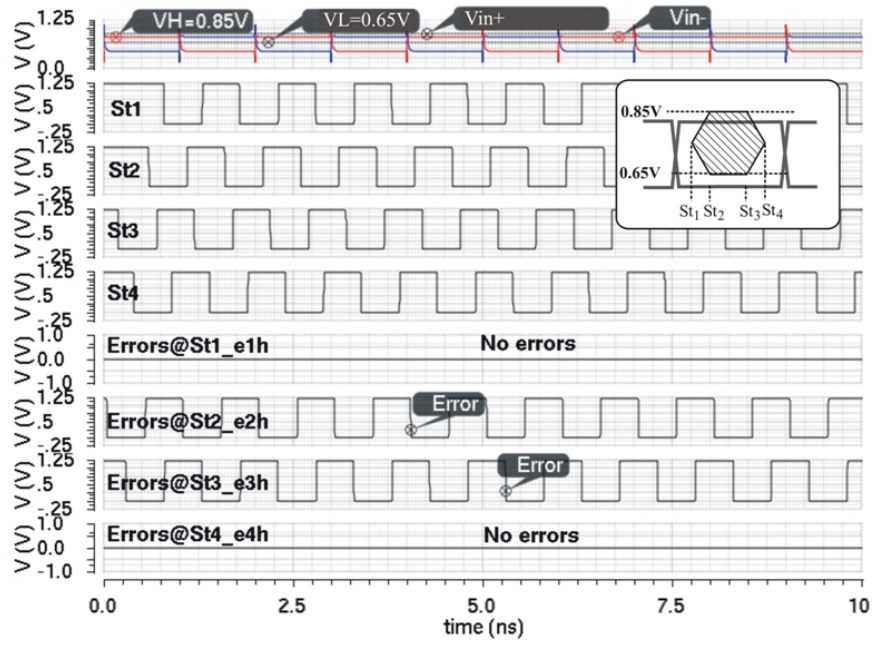

Figure 4.10: Simulated output of full hexagon EOM with $v_{i n, \max }<V_{H}$ and $v_{\text {in, } m i n}<V_{L}$. Errors are detected at $S t_{2}$ and $S t_{3}$. Top plot : Received data symbol. 2nd-5th plots : Sampling clocks $S t_{2} \sim S t_{4}$. 6th-9th plots : Error outputs $e 1 h \sim e 4 h$. 


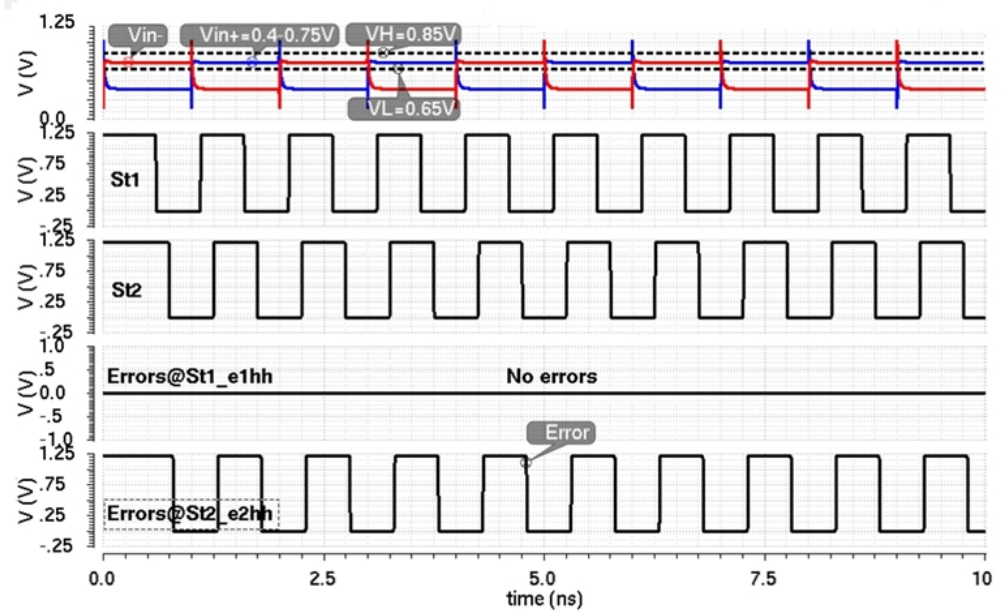

Figure 4.11: Simulated output of half hexagon EOM with $v_{\text {in, } \max }<V_{H}$ and $v_{\text {in,min }}<V_{L}$. Errors are detected at $S t_{2}$. Top plot : Received data symbol. 2nd-3rd plots : Sampling clocks $S t_{2} \sim S t_{3}$. 4th-5th plots : Error outputs $e 1 h \sim e 2 h$.

To further investigate the effect of channel loss on both EOMs, the length of the channel is increased to $200 \mathrm{~mm}$ and the response of the received data symbol, together with the output of the half hexagon and rectangular EOMs are shown in Fig.4.12 and Fig.4.13, respectively. It is seen that both EOMs provide the same error detection.

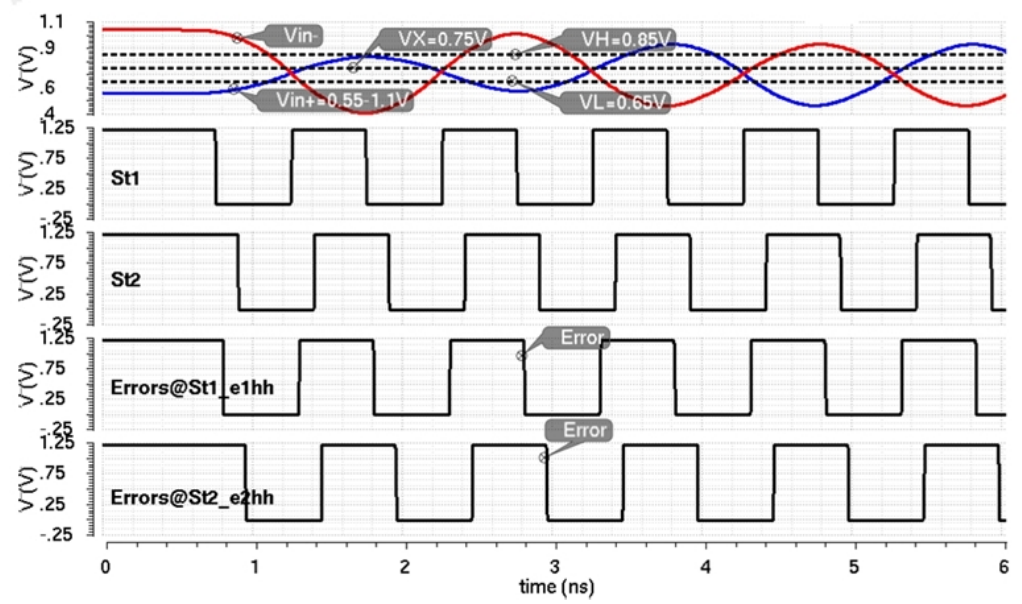

Figure 4.12: Simulated output of half hexagon EOM with a 200-mm channel. Top plot : Received data. 2nd and 3rd plots: Sampling clocks $S t_{2} \sim S t_{3}$. 4th and 5th plots: Output of half-hexagon EOM. 


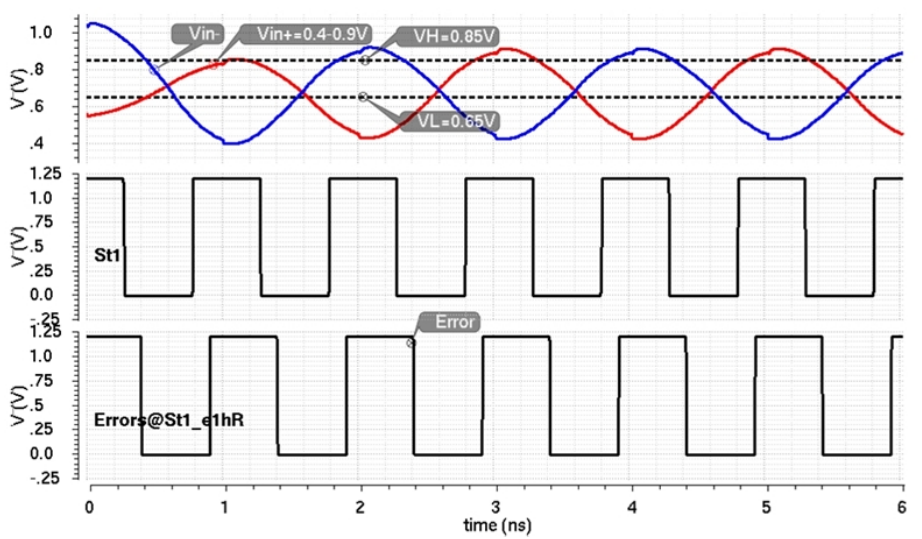

Figure 4.13: Simulated output of half rectangular EOM with a 200-mm channel. Top plot : Received data. 2nd plot: Sampling clock $S t_{1}$. 3rd plot : Output of half-rectangular EOM.

To investigate the effect of temperature variation and process spread on the performance of the proposed hexagon EOM, the data link with the proposed hexagon EOM was analyzed with temperature varied from $-20 \mathrm{C}$ to $80 \mathrm{C}$ and the the results are shown in Fig.4.14. The same data link was also analyzed at four process corners (at room temperature) and the results are provided in Fig.4.14.

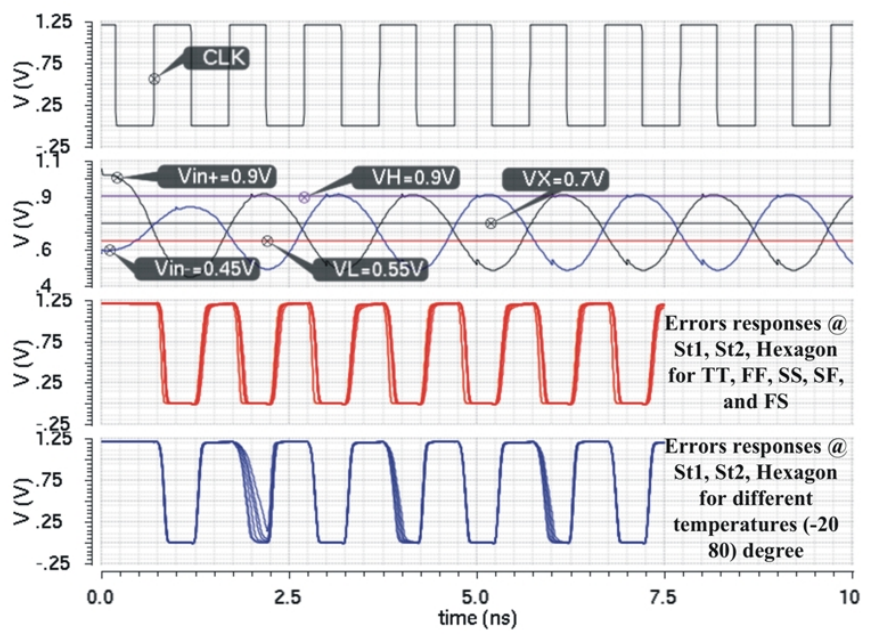

Figure 4.14: Simulated output of hexagon EOM with a 200-mm channel. Top plot : sampling clock. 2nd plot : Received data symbol and the minimum vertical eye-opening. 3rd plot : Output of EOM at process corners (TT: typical nMOS/typical pMOS; FF: fast nMOS/fast pMOS; FS: fast nMOS/slow pMOS, SF: slow nMOS/fast pMOS, and SS: slow nMOS/slow pMOS). Bottom plot : Output of EOM with temperture from $-20 \sim 80 \mathrm{C}$. 
Table 4.1: Reference comparison of proposed works

\begin{tabular}{||lllll||}
\hline Proposed works & Tech. & power consumption & Slicers & Step size \\
\hline \hline Hexagon EOM & $130 \mathrm{~nm}$ & $17.23 \mathrm{~mW}$ & 6 & Variable \\
Half Hexagon EOM & $130 \mathrm{~nm}$ & $9.72 \mathrm{~mW}$ & 3 & Variable \\
Maximum-Jitter EOM & $130 \mathrm{~nm}$ & $6.13 \mathrm{~mW}$ & 2 & Fix \\
\hline \hline
\end{tabular}

Table 4.2: Reference comparison of adaptive DFEs

\begin{tabular}{||lllll||}
\hline Performance & {$[30]$} & {$[34]$} & {$[68]$} & Maximum-jitter \\
\hline \hline Tech. & $130 \mathrm{~nm}$ & $130 \mathrm{~nm}$ & $130 \mathrm{~nm}$ & $130 \mathrm{~nm}$ \\
Data rate & $6.25 \mathrm{Gbps}$ & $6.25 \mathrm{Gbps}$ & $3.7 \mathrm{Gbps}$ & $2 \mathrm{Gbps}$ \\
Ch. length & $33 \mathrm{in}$ & $36 \mathrm{in}$ & $16 \mathrm{in}$ & $2 \mathrm{M}$ \\
Rx & CTLE/1-tap & 5 -tap & $3 \mathrm{IIR}$ & 2 -tap \\
power consumption & $180 \mathrm{~mW}$ & $14 \mathrm{~mW}$ & $17.3 \mathrm{~mW}$ & $15.45 \mathrm{~mW}$ \\
V.eye-opening & - & - & $45 \%$ & $1.0 \mathrm{~V}(83 \%)$ \\
H.eye-opening & - & - & - & $0.34 \mathrm{~ns}(68 \%)$ \\
Step size & Variable & Variable & Variable & Fix \\
Jitter & $1.3 \mathrm{ps}$ & - & $0.56 \mathrm{UI}$ & $80 \mathrm{ps}(16 \%)$ \\
\hline \hline
\end{tabular}

\subsection{Chapter Summary}

A power-efficient hexagon EOM was introduced and its design details were presented. As compared with full hexagon EOM, the proposed half hexagon EOM lowers the power and silicon consumption by $50 \%$, while performing the same functionality. Its performance was validated using simulations. 



\section{Chapter 5}

\section{Adaptive DFE Uing 2-Dimensional Hexagon EOM}

This chapter presents a variable step-size adaptive DFE utilizing a hexagon EOM for Gbps serial links. The adaptation specifically the step size used in search for the optimal tap coefficients of DFE is set by the severity of the violation of the pre-defined minimum eyeopening by received data symbols so as to achieve both a fast convergence of adaption and the maximum eye-opening. The effectiveness of the proposed adaptive DFE is evaluated by embedding it in a 2 Gbps serial link implemented in an IBM $130 \mathrm{~nm}$ 1.2V CMOS technology. For the purpose of comparison, an adaptive DFE with a rectangular EOM is also designed and included in the same data link. The data link with variable channel lengths is analyzed using Spectre from Cadence Design Systems with BSIM4 device models and simulation results are presented. The remaining of the chapter is organized as follows : Section 5.1 presents the principle work of the proposed adaptive DFE. Section 5.2 addresses the algorithm that utilizes to conrol the process of the adaptation. Section 5.3 details the circuit implementation of the proposed adaptive DFE. Simulation results that validate the

effectiveness of the proposed adaptive DFE are presented in Section 5.4. The chapter is summerised in Section 5.5. 


\subsection{The Principle}

Fig.5.1(a) shows the violation of the minimum eye-opening by the received data symbol at $t_{1}$ only. Fig.5.1(b) shows the violation of the minimum eye-opening at both $t_{1}$ and $t_{2}$. Since this case is more severe as compared with that of Fig.3(a), a higher level of the severity of the violation of the minimum eye-opening is defined. Since the violation of the minimum eye-opening of Fig.5.1(b) is more severe as compare with that of Fig.5.1(a), a larger fixed step should be warranted in search for the optimal DFE coefficients so as to provide a faster convergence time. When the severity of error drops, the step size is also reduced accordingly to lower the residue in computing tap coefficients.
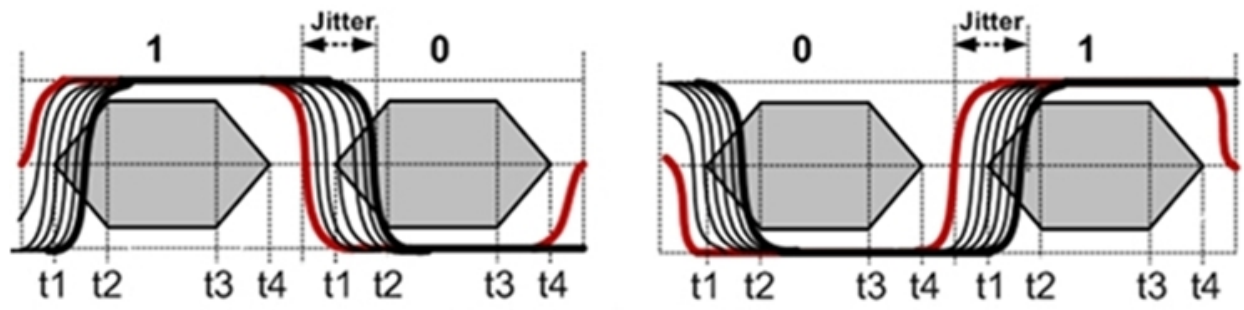

(a) Violation at $\mathrm{t} 1$ due to jitter
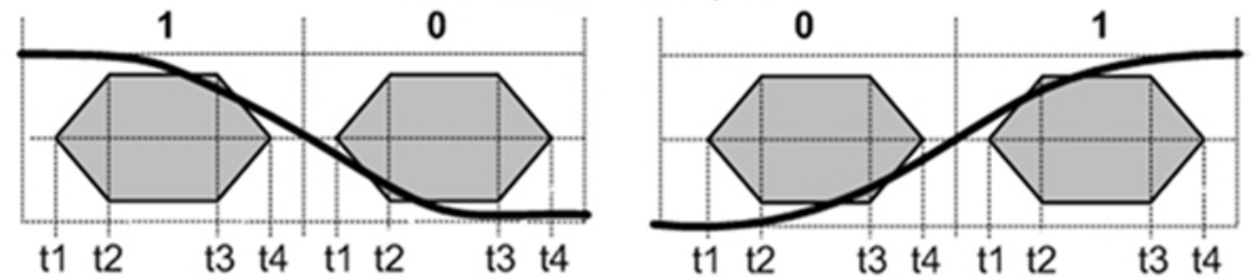

(b) Violations at 12 and 13 due to attenuation

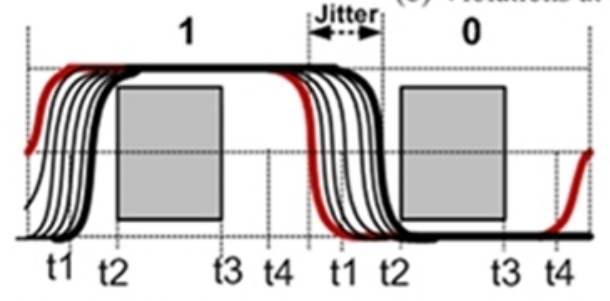

(c) No violations are recorded due to jitter

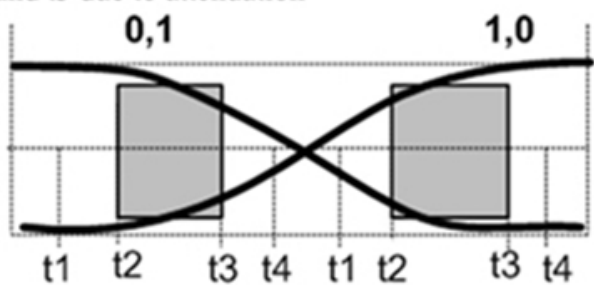

(d) Violations are recorded at 12 and $\mathrm{t} 3$ due to attenuation

Figure 5.1: (a) Violation of the minimum eye-opening at $t_{1}$ only. (b)Violation of the minimum eye-opening at $t_{1}$ and $t_{2}$. (c) No violation of the minimum hexagon EOM but violation of the minimum rectangular EOM exists. 


\subsection{The Algorithm}

In this work, the coefficients of the feedback taps of the DFE equalizer are obtained using the modified LMS depicted in Equation (5.1)

$$
C_{j, k+1}=C_{j, k}+h e_{k} D_{k}
$$

where $j=1,2, \ldots, N, N$ is the number of DFE taps, $C_{j, k+1}$ and $C_{j, k}$ are the coefficients of $j$-tap in steps $k+1$ and $k$ respectively, $h$ is the step size used to adjust the tap coefficients, $e_{k}$ is the error in step $k$, and $D_{k}$ is the decision in step $k$. It is seen that similar to SS-LSM DFE, the modified LMS DFE does not need the value of received data symbols. In stead, only the error generated by the error detect unit $e_{k}$ is needed. Depending upon the severity of the violation of the minimum eye-opening, different $e_{k}$ is assigned. A steepest ascent technique is employed to adjust tap coefficients. When an error is detected, the severity of error will be determined and fed to the adaptive engine. The adaptive engine will set the step size $h$ based on the severity of the error. The step size is set by adjusting the tail current of the taps based on the step size provided. The opening of the data eye will increase accordingly. This process will repeat until no more error is detected by the error detect unit. When this occurs, adaption is completed and the optimal tap coefficients are found. The flow-chart of the adaptive process is shown in Fig.5.2. 


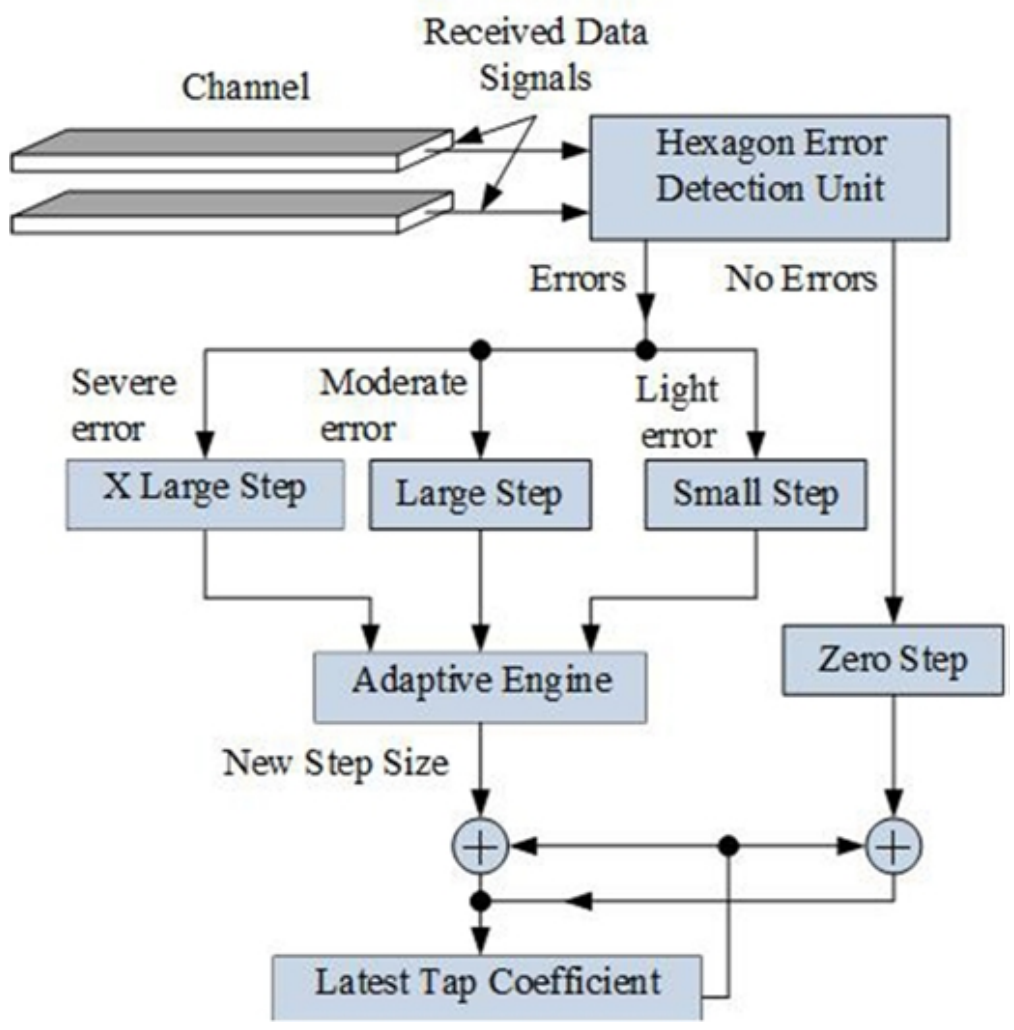

Figure 5.2: Search engine of proposed hexagon EOM based adaptive DFE.

\subsection{Implementation}

\subsubsection{The Architecture}

A DFE core with two feedback taps shown in Fig. 5.3 is designed in IBM $130 \mathrm{~nm} 1.2 \mathrm{~V}$ CMOS to demonstrate the effectiveness of the proposed adaptive DFE. The underlying principle can be applied to DFE with an arbitrary number of taps. A pre-amplifier with its load resistors implemented using PMOS transistors $M_{7,8}$ in triode is needed to amplify the received data symbol so that the input of the slicer is sufficiently large to minimize the possibility of the erroneous decision of the sliver. As the received symbol is often severely attenuated by the channel upon arriving at the far end of the channel, input offset voltage of the pre-amplifier mainly arising from the mismatches between input pair $M_{1}, M_{2}$ could be comparable to the 
received data symbols, resulting in changing the switching time (delay) of the comparator. Input offset voltage compensation is therefore required at the pre-amplification stage. As our goal of this study was to develop an EOM-based adaptive DFE, no input offset voltage cancellation was implemented in the design presented in the paper. Readers are referred to [25], [37], [66] for various techniques for input offset voltage compensation in Gbps serial links.

The output of the slicers is fed to a differential current-mode summer for tap 1 of the DFE. Its delayed version is fed to another differential current-mode summer for tap 2 of the DFE.

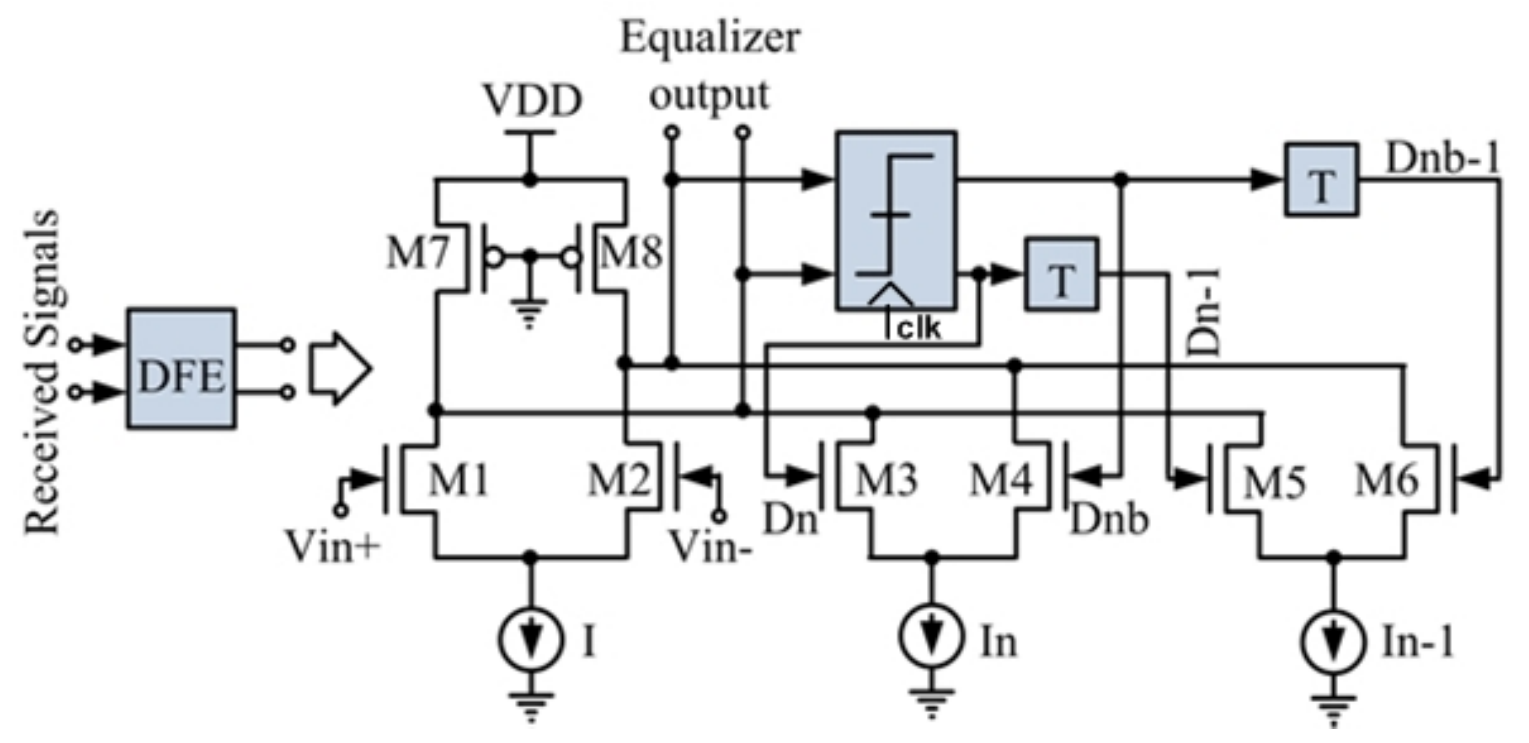

Figure 5.3: DFE core. Transistor sizes: $W_{1,2}=4 \mu \mathrm{m}, W_{3,4,5,6}=3 \mu \mathrm{m}, W_{7,8}=1 \mu \mathrm{m}, W_{9}=40 \mu \mathrm{m}$, and $W_{10,11}=30 \mu \mathrm{m}, L=0.13 \mu \mathrm{m}$. For all transistors biasing: $I_{s s}=2 \mu \mathrm{A}, V_{b}=0.8 \mathrm{~V}$.

The delay of tap 1 is provided by the intrinsic delay of the current-steering logic $M_{3} / M_{4}$ while the delay of tap 2 is provided by both the explicit delay stage and the intrinsic delay of the current-steering logic $M_{5} / M_{6}$. The output of the slicer is multiplied by an appropriate coefficient that ideally matches the voltage of the first post-cursor. Multiplication is performed by steering the tail current whose value is the coefficient of tap $1, C_{0}$. The delayed version 
of the output of the slicer is multiplied by an appropriate coefficient to match the voltage of the second post-cursor. The DFE eliminates the first two post-cursors by adjusting tap coefficients $C_{0}$ and $C_{1}$.

\subsubsection{Slicer}

The three-stage clocked comparator proposed in [63] and shown in Fig. 4.4 was adopted as the slicer. The 3-stage configuration of the slicer minimizes the effect of duty-cycle distortion [64] and kick-back [67]. The first stage is a clocked differential amplifier with a cross-coupled inverter pair load to amplify the differential symbol signal. The positive feedback formed by the cross-coupled inverter pair ensures the rapid transition of the output of the comparator. The output of the first stage is fed to a self-biased differential amplifier that performs further signal. The positive feedback formed by the cross-coupled inverter pair ensures the rapid transition of the output of the comparator. The output of the first stage is fed to a self-biased differential amplifier that performs further amplification and differential to single-ended conversion. The 3rd stage is simply a static inverter. The deployment of cross-coupled inverter pair in stage 1 ensures that the outputs of the stage 1 switch simultaneously regardless of the mismatches between $M_{1}$ and $M_{2}$, and that between $M_{3} / M_{4}$ inverter and $M_{5} / M_{6}$ inverter pair. Similarly, the self-biased differential pair in stage 2 will minimize duty-cycle distortion. Note that if the stage 1 was implemented using a differential pair, the mismatches between the input transistors of the differential pair would give rise to differential triggering voltages of the comparator.

\subsubsection{Error Detection Unit}

Adaptive DFE eliminates the ISI of the current symbol by adjusting the coefficients of the taps of the DFE based on the severity of the error. The control signal determining the step size in search for the optimal coefficients of DFE comes from the error detection unit 
(EDU). In the proposed adaptive DFE, a hexagon EOM is used to detect the violation of the minimum eye-opening of the received data symbols. Three threshold voltages $V_{H}, V_{X}$, and $V_{L}$ and two sampling points $t_{1}$ and $t_{2}$ are utilized to monitor the quality of received data eyes. Three clocked slicers are used to detect the violation of the minimum eye-opening, as shown in Fig.5.4.

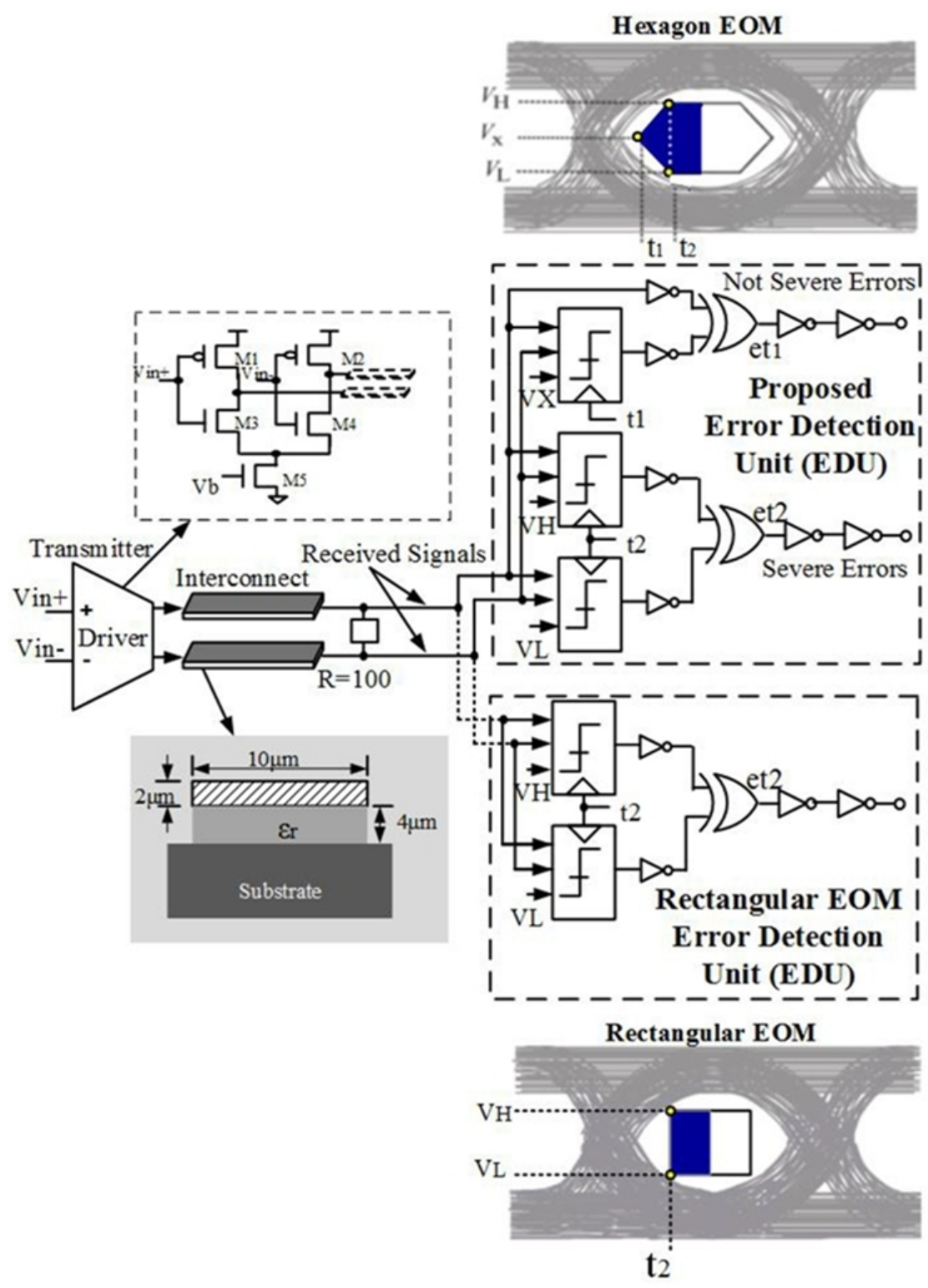

Figure 5.4: Error detection units. 
Three levels of the severity of the violation of the minimum eye-opening are provided by EDU, as shown graphically in Fig.5.5.

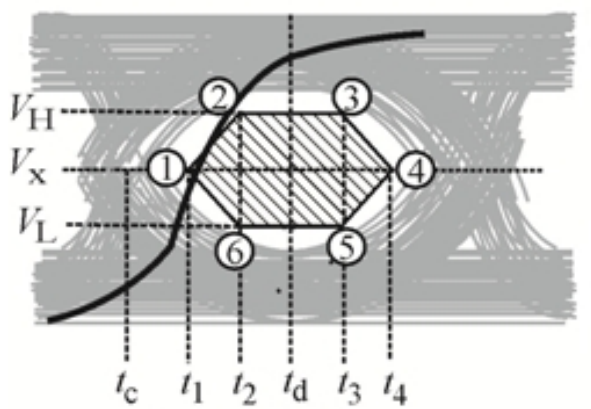

(a) Light violation

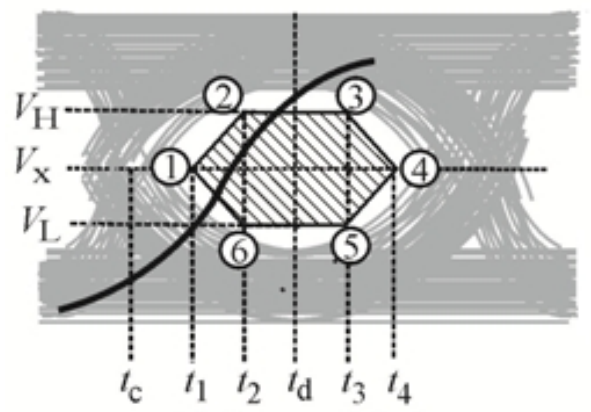

(c) Severe violation

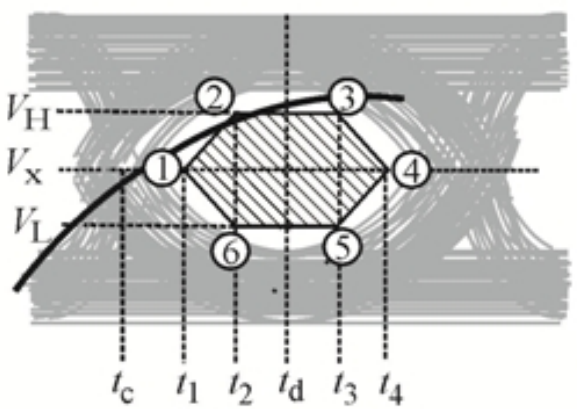

(b) Moderate violation

Figure 5.5: Severity of violation with hexagon EOM.

When the minimum eyeopening is violated at $t_{1}$, a light violation error flag will be set. When the minimum eye-opening is violated at $t_{2}$, a moderate violation error flag will be set, and when the minimum eyeopening is violated at both $t_{1}$ and $t_{2}$, a severe violation error flag will be set. Clearly for the different severity of violation, the action provided by DFE will differ accordingly. 


\subsubsection{Digital-To-Analog Converter}

The configuration and schematic of variable step-size digital-to-analog converter (DAC) are shown in Fig.5.6. Two charge pumps that provide two different currents are used for tap 1 and tap 2. The charge pump formed by $M_{3}-M_{8}$ will be in action when a not severe error
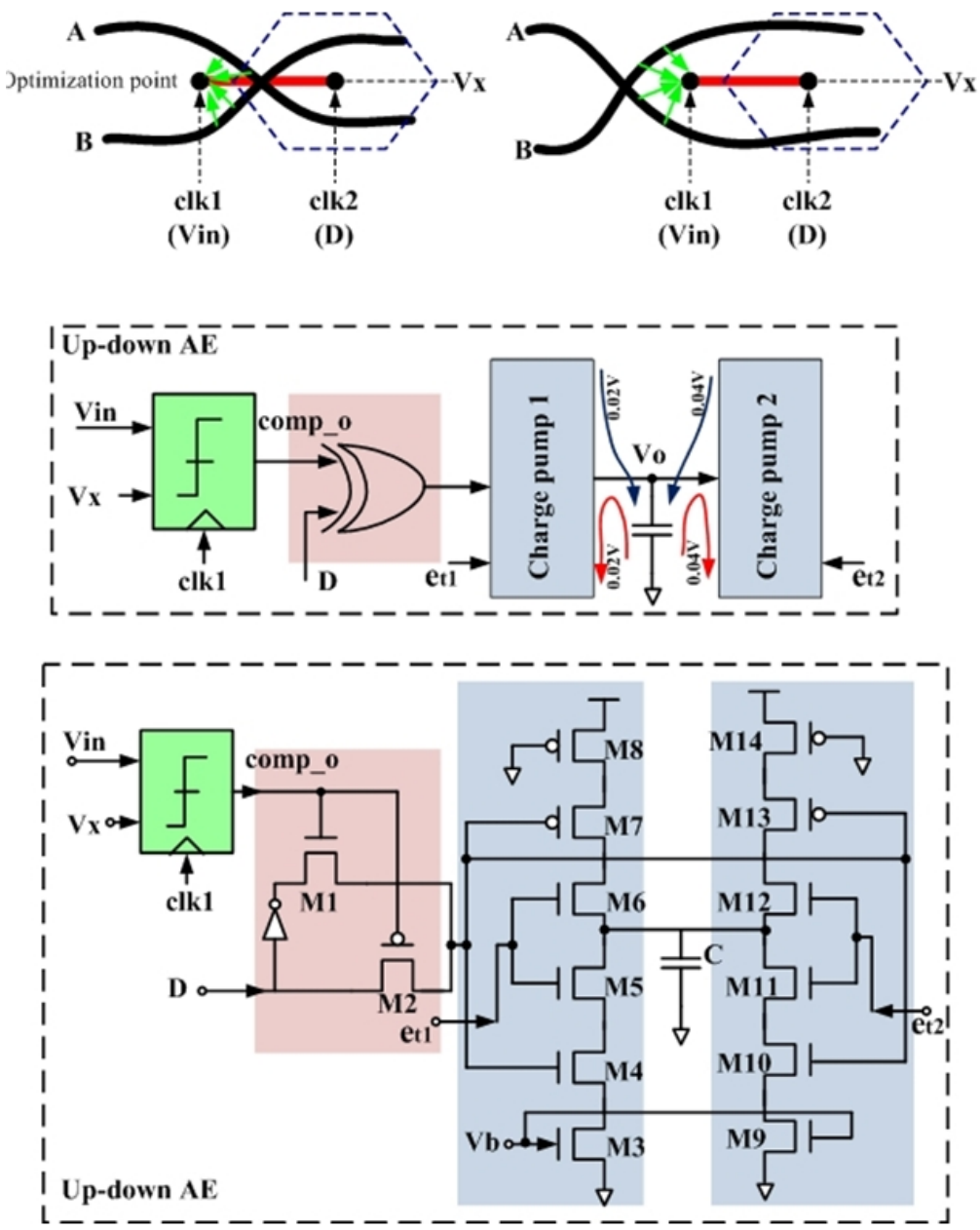

Figure 5.6: Configuration (top) and schematic (bottom) of variable step-size DAC. Charge pump $M_{3}-M_{8}$ is enabled when $e_{t 1}=1$. Charge pump $M_{9}-M_{14}$ is enabled when $e_{t 2}=1$.

$\left(e_{t 1}\right)$ is flagged. Both charge pumps will be ON when a severe error is detected $\left(e_{t 1}=\right.$ $e_{t 2}=1$ ). In the latter case, the voltage of the capacitor will change much faster. The DAC maps the severity of error to different voltages with a voltage step $20 \mathrm{mV}$. The voltage step 
is chosen in consideration of the corresponding change of the tail current of the feedback taps of the DFE. If the step is too small, the action of the DFE will be too weak to be

effective. On the other hand, if the step is too large, the action of the DFE will be overly strong, resulting in an unwanted oscillation in search for optimal DEF coefficients subsequently a long adaption process. A $10 \mathrm{pF}$ capacitor is used at the output of the charge pump. The value of the capacitor must also be chosen with care. If the capacitance is too large, the output voltage will not change fast enough to provide an appropriate voltage to DFE, resulting in a slow adaption. On the other hand, if the capacitance is too small, a large voltage fluctuation will exist, which will in turn have a negative effect on the search for the optimal coefficients of DFE. In order to allow the voltage of the capacitor to change in either positive or negative directions, depending upon the nature of the violation of the minimum eye-opening, two slicers, one at the edge of the data symbol and the other at the center of the data symbol, and a XOR2 gate are employed.

Consider the data symbol A shown in Fig.5.6(top-left). the slicer at $c l k_{1}$ outputs 1 and that at $c l k_{2}$ outputs 0 . As a result, the XOR2 gate sends 1 to the charge pump and the voltage of the capacitor of the charge decreases accordingly. Now, let us consider the data symbol A shown in Fig.5.6(top-right). Since the slicer at $c l k_{1}$ outputs 0 and that at $c l k_{2}$ outputs 0 , the XOR2 gate sends 0 to the charge pump and the voltage of the capacitor of the charge increases accordingly.

\subsubsection{XOR Gate}

The schematic of the current-mode differential XOR2 gate is shown in Fig.4.6. Current-mode logic is known for its high speed and low switching noise generation. To improve the speed of the circuit, the load resistors are replaced with two PMOS transistors $M_{7}, M_{8}$ working in the triode region. 


\subsection{Simulation Results}

For the purpose of comparison, a rectangular EOM-based adaptive DFE was also designed, as shown in Fig.5.4. The proposed adaptive DFE shown in Fig.5.7 consists of a main amplifier for main cursor, a slicer, three delay cells, two feedback taps, an error detection unit, and a DAC. Two serial links with the same transmitter and channel but different error detection units, specifically, a proposed adaptive DFE with a hexagon EOM and an adaptive DFE with a rectangular EOM, were designed in an IBM $130 \mathrm{~nm} 1.2 \mathrm{~V}$ CMOS technology and analyzed using Spectre from Cadence Design Systems with BSIM4 device models.

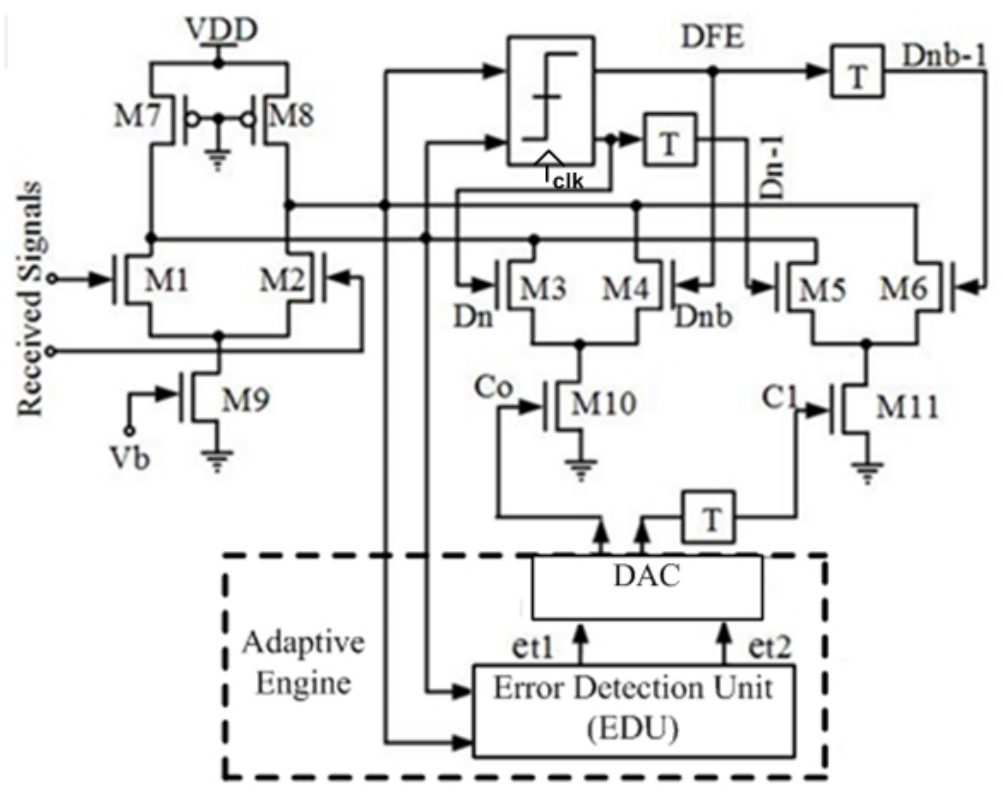

Figure 5.7: Proposed adaptive DFE.

The data rate of the link is 1 Gbps. A differential current-mode driver conveying a 2 $\mathrm{mA}$ current to the channel was employed. The channel is a transmission line terminated with a $100 \Omega$ resistor. Various channel lengths are used to generate different levels of the severity of violation of the minimum EOM. Two differential current-mode taps are employed to eliminate the first two post-cursors of received data symbols. The tail currents of the 
feedback taps are adjusted by the adaptive DFE. The output of the error detection unit is connected to the input of the adaptive engine to update the tail current of the feedback taps of the adaptive DFE. Fig.5.8 shows the response of channels of length $35 \mathrm{~mm}$ and $55 \mathrm{~mm}$ to a pulse input which is applied to the near end of the channels, respectively. The dispersion of the input pulse due to the finite bandwidth of the channels is evident. The longer the channel, the severe the dispersion. When channel length is small, the voltage swing of the received data was observed to be $0.45 \mathrm{~V} \sim 1.1 \mathrm{~V}$. The minimum vertical opening of the eye was therefore set to $V_{H}=0.95 \mathrm{~V}, V_{X}=0.75 \mathrm{~V}$, and $V_{L}=0.55 \mathrm{~V}$.
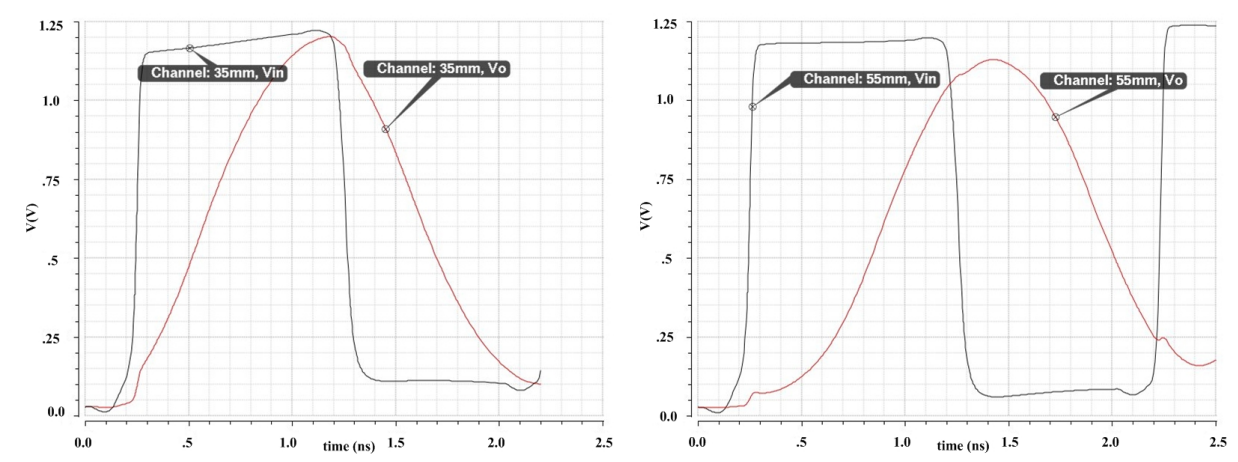

Figure 5.8: Response of channels of length $35 \mathrm{~mm}$ (left) and $55 \mathrm{~mm}$ (right) to a pulse input at the near end of the channel.

The sampling times for hexagon were set to $t_{1}=0.2 \mathrm{~ns}$ and $t_{2}=0.4 \mathrm{~ns}$ while for the rectangular EOM, it was set to $t_{1}=0.4 \mathrm{~ns}$. The simulation results of tap coefficients for the adaptive DFE with a hexagon EOM and the adaptive DFE with a rectangular EOM are compared in Fig.5.9.

The waveforms represent the signals at the output of the adaptive engine. The waveform of the coefficients of the adaptive DFE with a hexagon EOM shows four different types of errors, i.e., no errors, light errors, moderate errors, and severe errors.

Fig.5.10 shows the errors of the adaptive DFE with a hexagon EOM and those of the adaptive DFE with a rectangular EOM. The top waveform represents moderate errors detected at $t_{2}$. The waveform in the middle represents light errors detected at $t_{1}$. The last waveform shows the errors detected by the adaptive DFE with a rectangular EOM. It is 


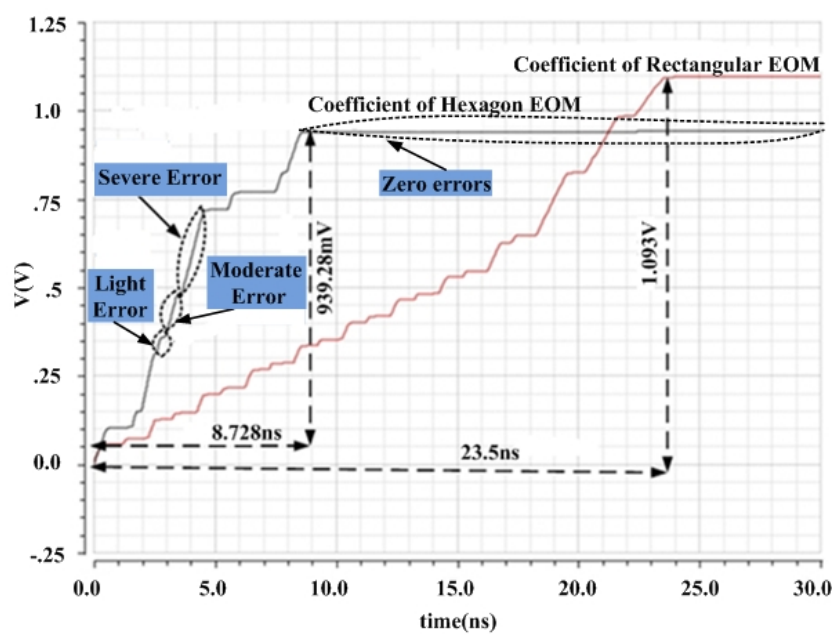

Figure 5.9: Tap coefficients of adaptive DFE with hexagon and rectangular EOMs.

observed that a large number of errors are generated when the rectangular EOM is used, resulting in a long sequence of unnecessary DFE actions subsequently a slow convergenceand high power consumption.

It is evident from Fig.5.9 and Fig.5.10 that the variable step sizes provided by the proposed adaptive DFE with a hexagon EOM enable the DFE to converge in 8.7 ns over voltage range $939.2 \mathrm{mV}$. As compared with convergence time $23.5 \mathrm{~ns}$ and voltage range $1.09 \mathrm{~V}$ of the adaptive DFE with a rectangular EOM,

the proposed adaptive DFE with a hexagon EOM converges approximately 4 times faster. The improved convergence speed is mainly due to (i) the use of large step sizes in search for optimal tap coefficients when the violation of the minimum eye-opening is severe and (ii) the reduced step size when searching approaches the optimal tap coefficients. table tabulates the performance of the proposed EOM-based adaptive DFE.

Fig.5.11 shows the waveform of data symbols at the far end of the channel before and after the proposed adaptive DFE. The increased opening of the data eye after adaptive equalization is evident, especially when the channel length is large.

Figs.5.12 and Figs.5.13 plot the adaptation process with channel lengths $35 \mathrm{~mm}$ and 55mm, 


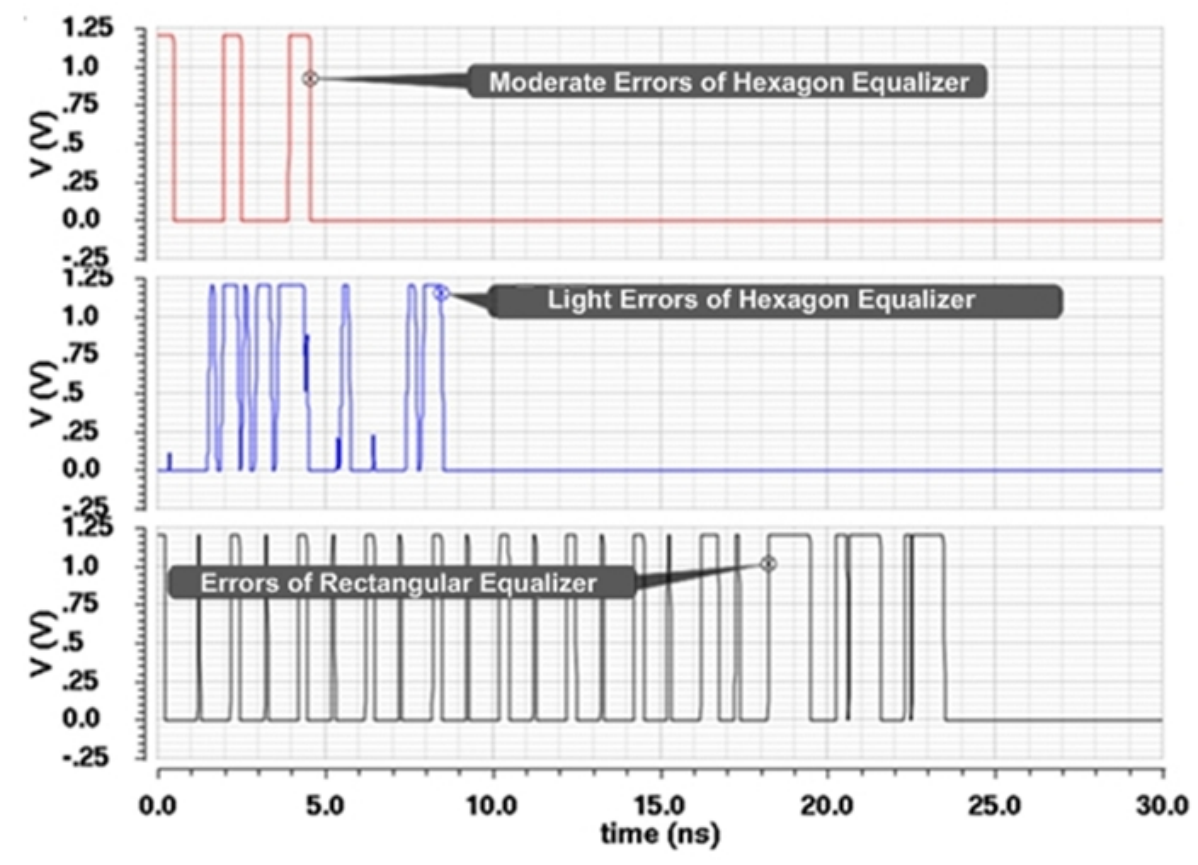

Figure 5.10: Error detection of rectangular and hexagon adaptive DFE.

respectively.

It is observed that tap1 and tap2 have different adjustment rates set by the two charge pumps depicted earlier. Also observed that initially both the violation of the minimum eyeopening and the XOR operation of the samples at $c l k_{1}$ and $c l k_{2}$ of Fig.5.4 yield up and down adjustments of the tap coefficients, speeding up the adaptation process. With the increase in the eye-opening, no violation of the minimum eye-opening is detected at $24 \mathrm{~ns}(35 \mathrm{~mm}$ channel) and $79 \mathrm{~ns}(55 \mathrm{~mm}$ channel). This also removes the effect of the output of the XOR operation, resulting in stable tap coefficients.

Table 5.1 illustrates the performance of proposed EOM-based adaptive DFE.

\subsection{Chapter Summary}

A variable step-size adaptive DFE utilizing a hexagon EOM was proposed for Gbps serial links. The proposed adaptive DFE provides three levels of errors, namely light error, 

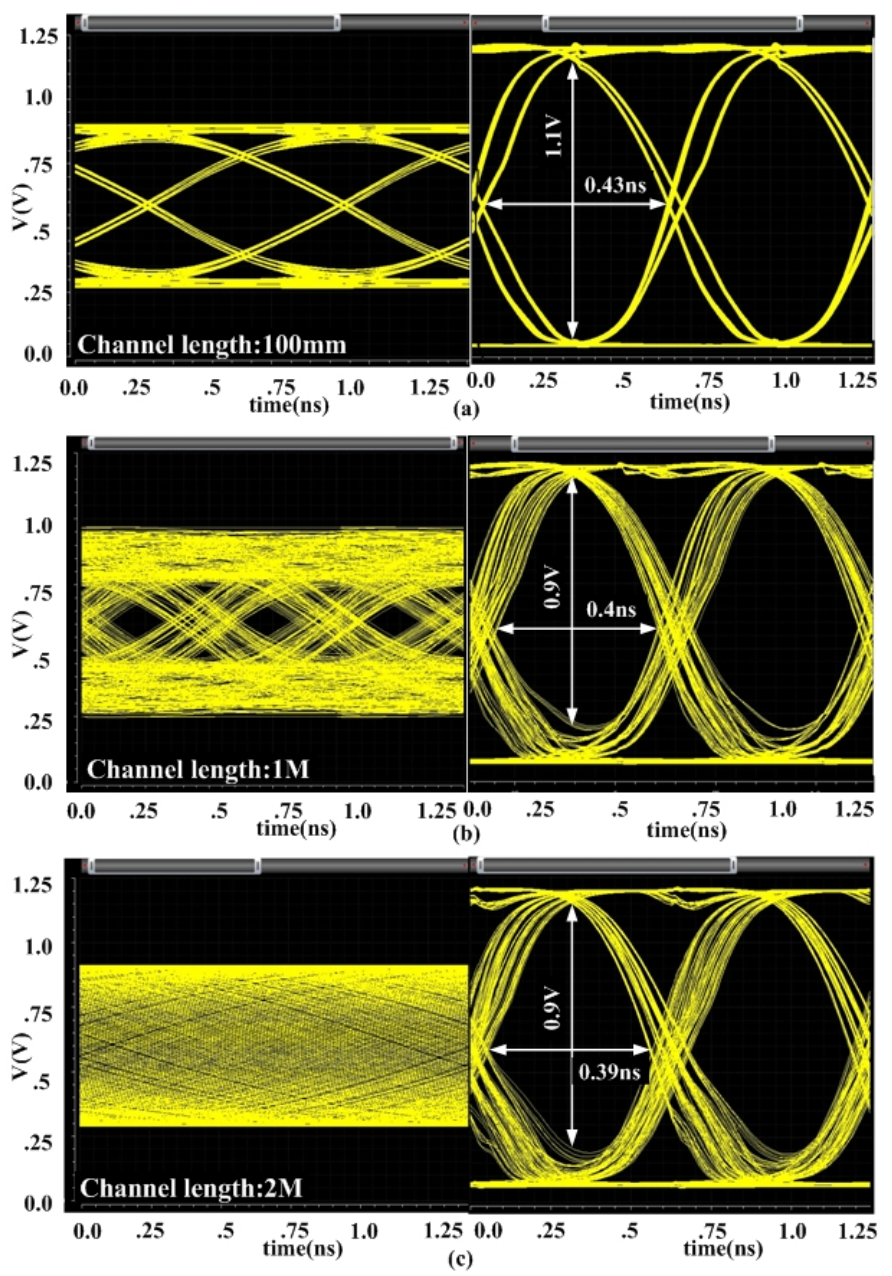

Figure 5.11: Waveform of data before (left) and after (right) the proposed adaptive DFE. Top: channel length $=100 \mathrm{~mm}$. Middle: channel length=500mm. Bottom: channel length=1M.

moderate error, and severe error. These errors are fed to a current-mode DAC to generate a variable step-size voltage to update the tap coefficients and achieve the fast convergence time of adaption. As compared with an adaptive DFE using a rectangular EOM, the proposed adaptive DFE reaches the convergence time approximately 4 times faster. The effectiveness of the proposed adaptive DFE was evaluated by embedding it in a 2 Gbps serial link implemented in an IBM $130 \mathrm{~nm}$ 1.2V CMOS technology. 


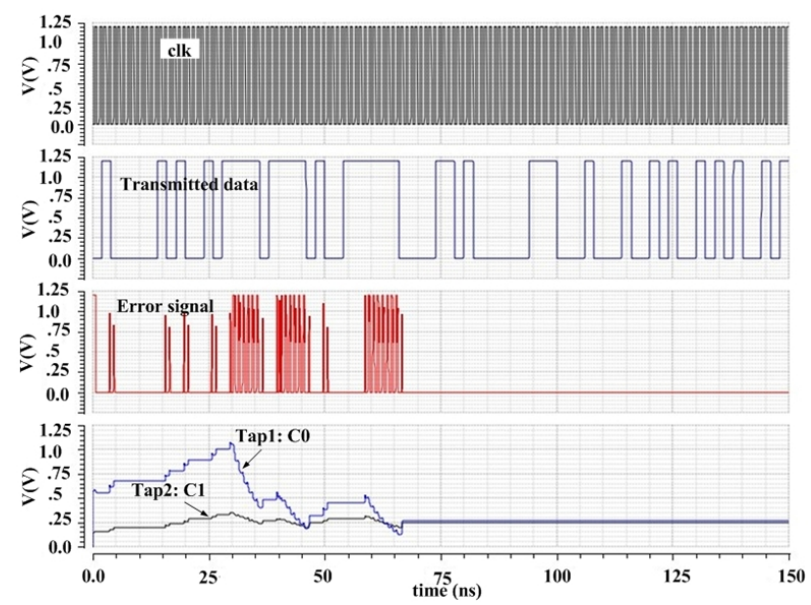

Figure 5.12: Error detection and tap coefficients of proposed adaptive DFE with $35 \mathrm{~mm}$ channel length.

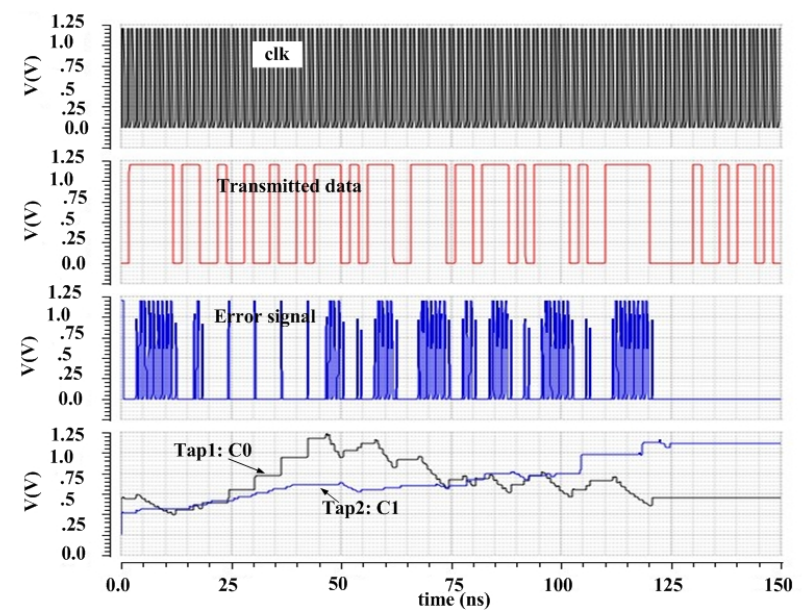

Figure 5.13: Error detection and tap coefficients of proposed adaptive DFE with $55 \mathrm{~mm}$ channel length. 
Table 5.1: Performance of proposed hexagon eye-opening monitor adaptive DFE (2 Meter FR4 channel).

\begin{tabular}{ll}
\hline Tech. & IBM $130 \mathrm{~nm}$ \\
Supply voltage & $1.2 \mathrm{~V}$ \\
Data rate & $2 \mathrm{Gbps}$ \\
Power of charge pumps & $4.13 \mathrm{~mW}$ \\
Power of error detect units & $7.54 \mathrm{~mW}$ \\
Power of DFE core (pre-amp., tap generators, delay units, ...) & $5.71 \mathrm{~mW}$ \\
Total power & $17.38 \mathrm{~mW}$ \\
Vertical eye-opening & $0.9 \mathrm{~V}(75 \%)$ \\
Horizontal eye-opening & $0.39 \mathrm{~ns}(78 \%)$ \\
Jitter & $55 \mathrm{ps}(11 \%)$ \\
\hline
\end{tabular}





\section{Chapter 6}

\section{Maximum-Jitter Adaptive DFE}

This chapter presents a maximum jitter adaptive DFE for Gbps serial links. The adaptation in search for the optimal tap coefficients of DFE is set by the detection of the violation of the maximum jitter of received data symbols. The effectiveness of the proposed adaptive DFE is evaluated by embedding it in a 1 Gbps serial link with a micro-strip channel of variable length implemented in an IBM $130 \mathrm{~nm}$ 1.2V CMOS technology. The data link with variable channel length is analyzed using Spectre from Cadence Design Systems with BSIM4 device models. Simulation results are presented. The remaining of the chapter is organized as follows : Section 6.1 details the proposed maximum jitter adaptive DFE. Implementation of the proposed jitter-based adaptive DFE is addressed in Section 6.2. Simulation results that validate the effectiveness of the proposed adaptive DFE are presented in Section 6.3. Section 6.4 investigates the drawbacks of adaptive DFE utilizing SS-LMS and EOM. The proposed adaptive DFE that outperforms an adaptive DFE using SS-LMS and EOM is addressed in Section 6.5. The simulation results are given in Section 6.6. The chapter is concluded in Section 6.7. 


\subsection{Maximum-Jitter Adaptive DFE}

\subsubsection{The Principle}

In the proposed jitter-based adaptive DFE, the detection of whether a violation of the maximum jitter at the edges of received data symbols is performed by comparing the voltage of the received data symbol with its common-mode voltage $V_{x}$ at $t_{1}$ and $t_{2}$ where $t_{1}=t_{c}+\Delta t_{c}$, $t_{c}$ is the jitter-free threshold-crossing time, $\Delta t_{c}$ is the maximum jitter allowed, and $t_{2}$ is the time of the center of the data symbol, as shown in Fig.6.1.

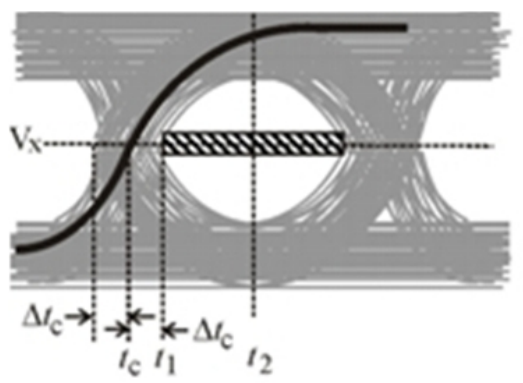

(a)

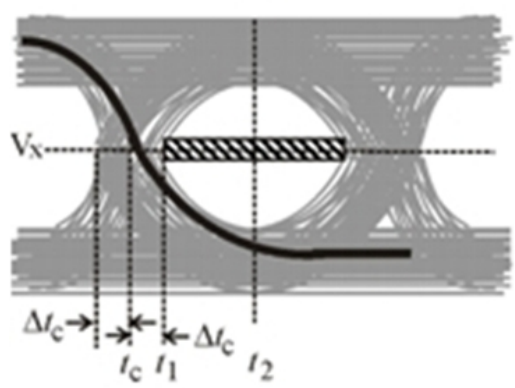

(c)

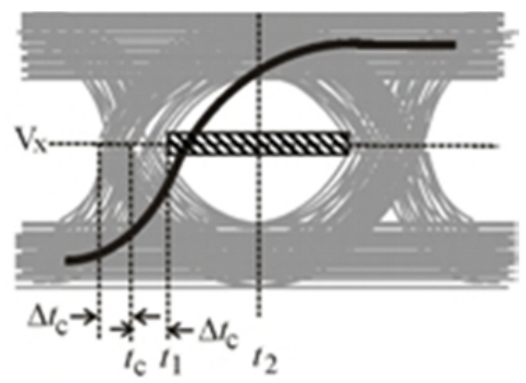

(b)

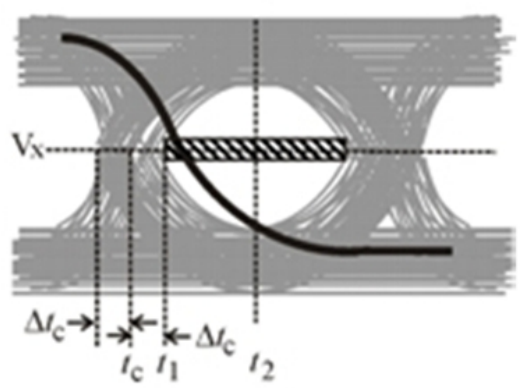

(d)

Figure 6.1: Jitter-based of error detection. (a,c) - No error exists. (b,d) - Errors exist.

The following cases are examined:

- Case 1 (Fig.6.1(a)): No error is detected because $V_{s}\left(t_{1}\right), V_{s}\left(t_{2}\right)>V_{x}$.

- Case 2 (Fig.6.1(b)): An error is detected because $V_{s}\left(t_{1}\right)<V_{x}$ and $V_{s}\left(t_{2}\right)>V_{x}$. 
- Case 3 (Fig.6.1(c)): No error is detected because $V_{s}\left(t_{1}\right), V_{s}\left(t_{2}\right)<V_{x}$.

- Case 4 (Fig.6.1(d)) An error is detected because $V_{s}\left(t_{1}\right)<V_{x}$ and $V_{s}\left(t_{2}\right)>V_{x}$.

An error signal indicating whether a violation of the maximum allowable jitter occurs or not can therefore be generated by XORing the output of two comparators, one clocked at $t_{1}$ and comparing $V_{s}\left(t_{1}\right)$ with $V_{x}$ and the other clocked at $t_{2}$ and comparing $V_{s}\left(t_{1}\right)$ with $V_{x}$. The tap coefficients of DFE are obtained using the following modified LMS algorithm

$$
C_{j, k+1}=C_{j, k}+h e_{k} D_{k},
$$

where $j=1,2, \ldots, N, \mathrm{~N}$ is the number of DFE taps, $C_{j, k+1}$ and $C_{j, k}$ are the coefficients of tap- $j$ in steps $k+1$ and $k$, respectively, $h$ is the step size used to adjust the tap coefficients, $e_{k}$ is the error detected in step $k$ using the error detect unit (EDU) depicted earlier, and $D_{k}$ is the decision of the slicer in step $k$. It is seen that similar to SS-LMS DFE, the modified LMS DFE does not require the value of received data symbols. Instead, only the error generated by the EDU is needed. When an error is detected, the error signal will be fed to the adaptive engine, as shown in Fig.6.2. The adaptive engine will set $h$ and adjust the tap coefficients of DFE. This process continues until no error is detected. When this occurs, the adaptation process completes and the optimal tap coefficients that satisfy the specified worst jitter constraint are obtained. 


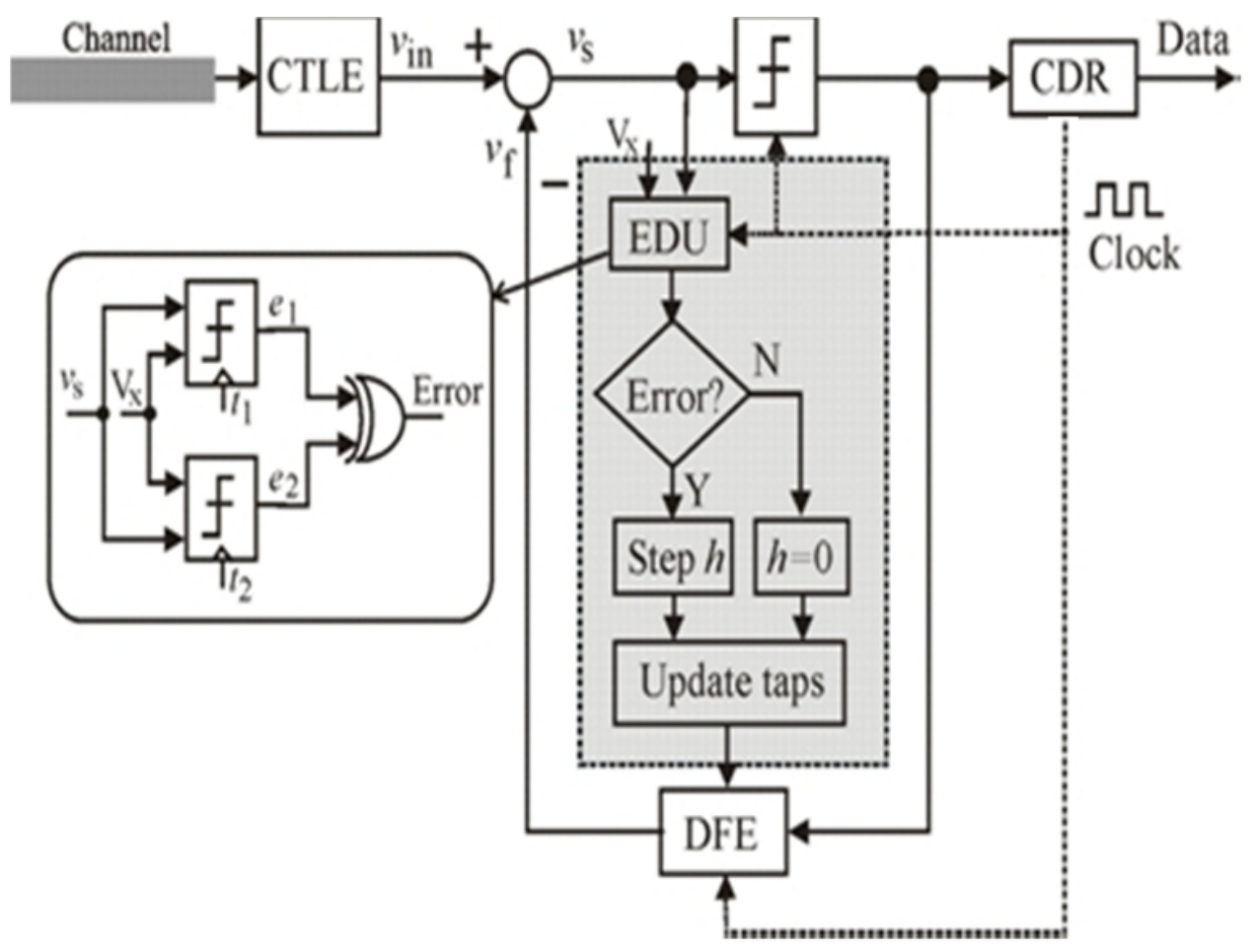

Figure 6.2: Adaptive engine of proposed jitter-based adaptive DFE.

\subsection{Implementation}

\subsubsection{Error Detection Unit}

The proposed jitter-based adaptive DFE adjusts the tap coefficients of DFE based on whether there is a violation of the maximum allowable jitter or not. The error detect unit consists of two clocked slicers that compare the voltage of incoming data symbol with its commonmode voltage at $\mathrm{t} 1$ and $\mathrm{t} 2$. The output of the comparators are XORed to generate the error signal, as shown in Fig.6.3. The error signal is then fed to the adaptive engine to produce an appropriate voltage that adjusts the tail currents of the tap generators. The XOR gate employed in the implementation is the schematic of the current-mode XOR2 gate shown in Fig.4.6. 


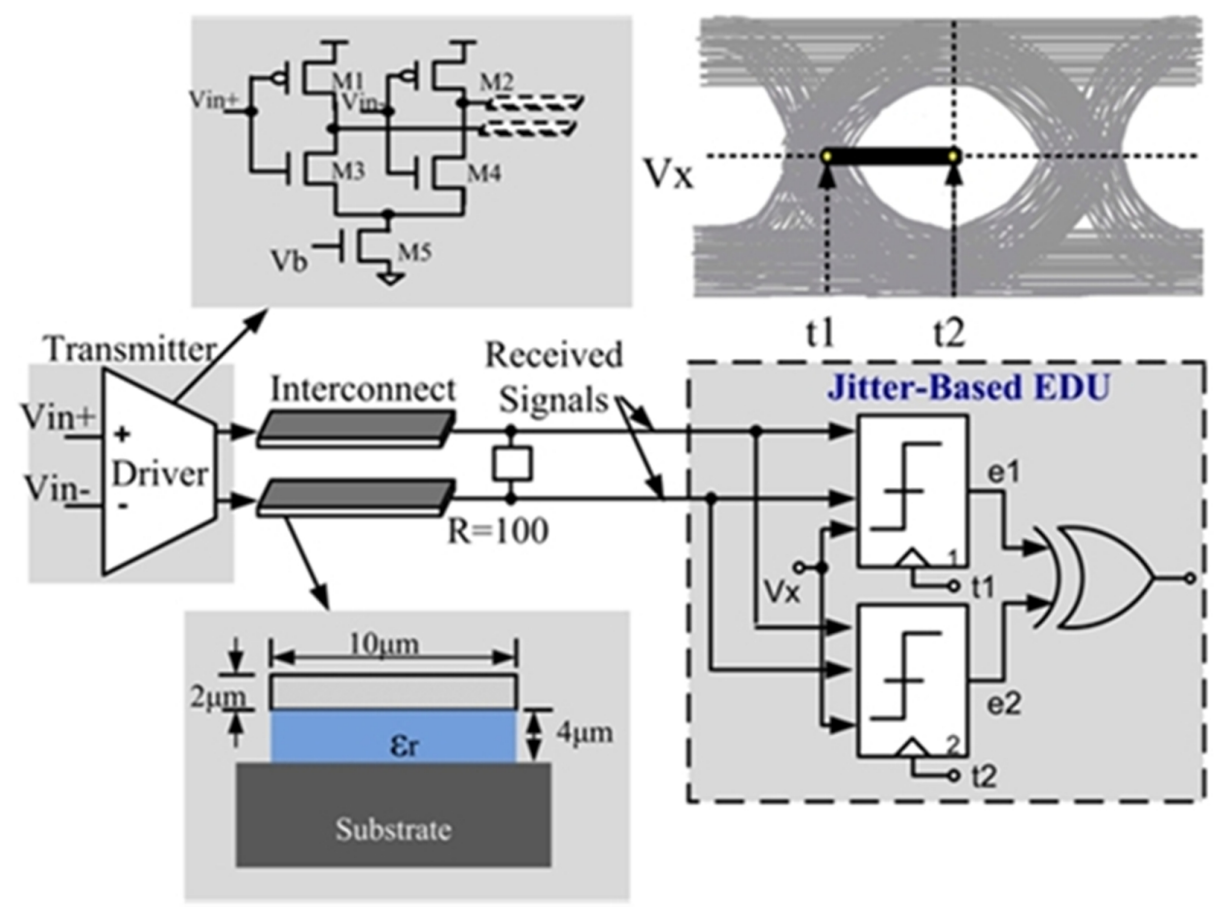

Figure 6.3: Error Detection Unit. Channel parameters : Microstrip with width $10 \mu \mathrm{m}$, height 2 $\mu \mathrm{m}$, and length $80 \mu \mathrm{m}$. Dielectric constant of field oxide : $\epsilon_{r}=5$.

\subsubsection{Digital-to-Analog Converter}

The one-bit digital-to-analog converter (DAC) shown in Fig.6.4 is used to map the Boolean error signal to a voltage. When no error is generated by the EDU, $M_{1}$ is off and the output voltage is set by the accumulated voltage across the capacitor. When an error is generated by the EDU, the output voltage is set by the accumulated voltage across the capacitor plus the voltage feed to the capacitor through $V_{d d}$. Therefore, the output increment. The output voltage increment is set to $20 \mathrm{mV}$. The voltage increment is chosen in consideration of the corresponding change of the tail current of the feedback taps of the DFE. If the step is too small, the action of the DFE will be too weak to be effective. 


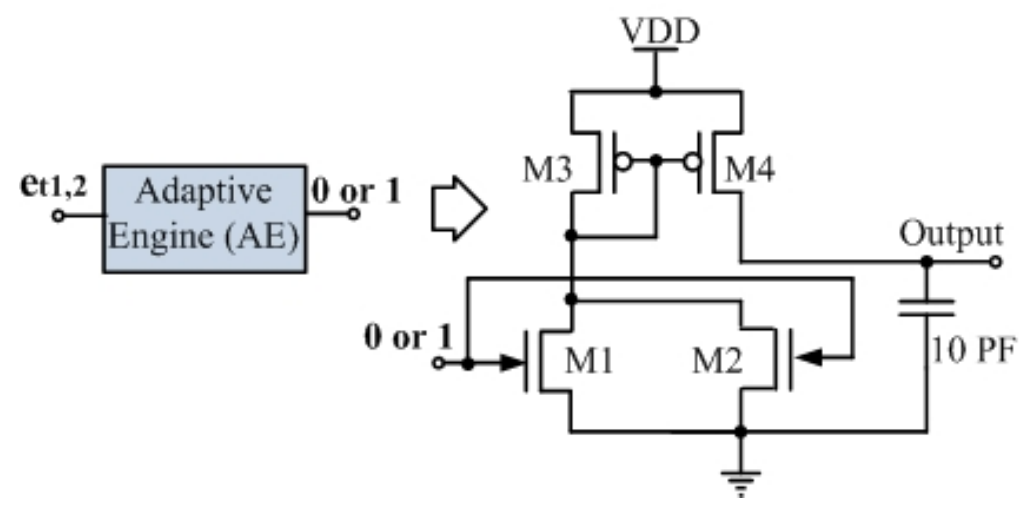

Figure 6.4: Schematic of digital-to-analog converter (DAC).

On the other hand, if the step is too large, the action of the DFE will be overly strong, resulting in an unwanted oscillation in search for optimal DEF coefficients. A 10 pF capacitor is employed at the output of the DAC. The value of the capacitor must also be chosen with care. If the capacitance is too large, the output voltage will not change fast enough to provide an appropriate voltage to DFE, resulting in a slow adaptation process. On the other hand, if the capacitance is too small, a large voltage fluctuation will exist, which will in turn have a negative effect on the search for the optimal coefficients of DFE.

\subsection{Simulation Results}

The effectiveness of the proposed jitter-based adaptive DFE is verified using a 1 Gbps serial link over an $80 \mu \mathrm{m}$ interconnect. The dimensions of the interconnect are given in Fig.6.3. The configuration of the proposed adaptive DFE is the same used in Fig.5.7 consists of a main amplifier for the main cursor, a slicer, two delay cells, two current-steering feedback taps, an error detection unit, and an adaptive engine. It is designed in an IBM $130 \mathrm{~nm} 1.2$ V CMOS technology and analyzed using Spectre from Cadence Design Systems with BSIM4 device models. A differential current-mode driver conveying a $2 \mathrm{~mA}$ current to the channel is employed. The channel is an $80 \mu \mathrm{m}$ transmission line terminated with a $100 \Omega$ resistor 
to reduce the effect of signal reflections. Two differential current-mode taps are employed to eliminate the first two post cursors of received data symbols. The tail currents of the feedback taps are adjusted by the adaptive DFE. When channel length is small (2 mm), the voltage swing of the received data was observed to be $0.45-1.1 \mathrm{~V}$. The threshold voltage is set to $V_{x}=0.75 \mathrm{~V}$ and two sampling times $t_{1}=0.1 \mathrm{UI}$ and $t_{2}=0.5 \mathrm{UI}$ are used in simulation. Fig.6.5 shows the adaptation process of the proposed adaptive DFE for $20 \mathrm{~mm}$ channel. It is observed that the adaptation process completes in less than $10 \mathrm{~ns}$ when no error is detected.

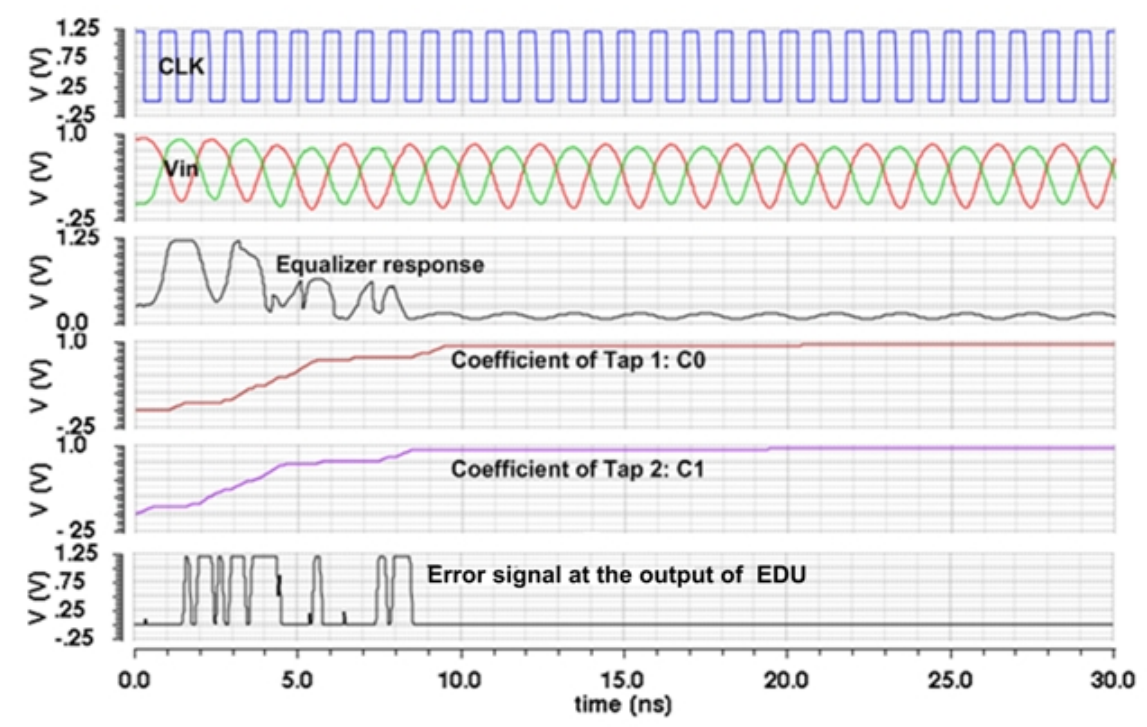

Figure 6.5: Adaptation process of proposed jitter-based adaptive DFE with a $20 \mathrm{~mm}$ channel.

Figs.6.6 shows the waveform of data symbols at the input of the slicer without (left ) and with (right) the proposed jitter-based DFE for (a) $1000 \mathrm{~mm}$, (b) 1M, and (c) $2 \mathrm{M}$ channel lengths, respectively. The data conveyed to the channel by the transmitter is a square wave. It is evident that the proposed adaptive DFE is capable of maximizing the opening of data eyes even if received data eyes are totally closed. 

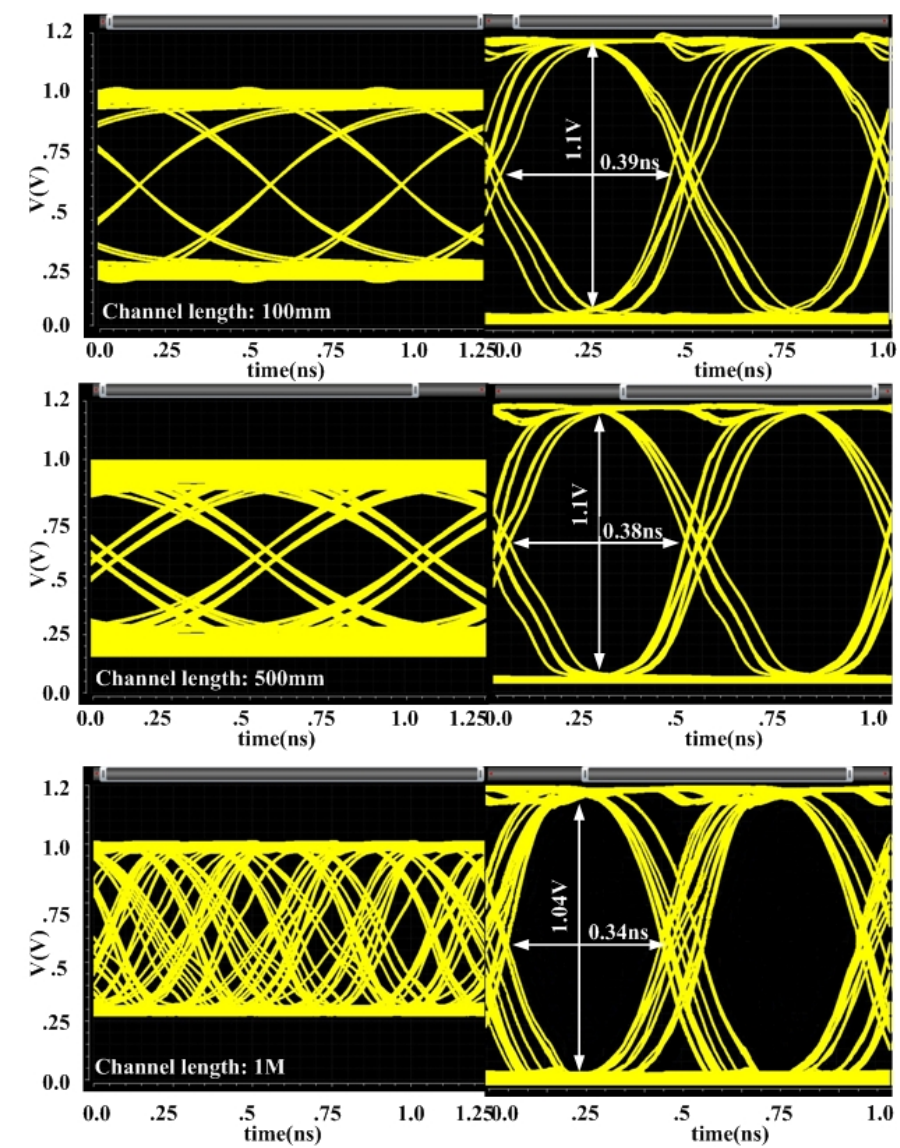

Figure 6.6: Waveforms of data symbols without (left) and with (right) the proposed 2-tap jitterbased adaptive DFE. Channel lengths : (a) $100 \mathrm{~mm}$, (b) 1M, and (c) $2 \mathrm{M}$.

It is seen that the proposed adaptive DFE is capable of opening the data eye. Fig.6.7 (a) shows the simulated eye diagram of data symbols at the input of the slicer without and with the proposed jitter-based DFE with channel length $50 \mathrm{~mm}$. Fig.6.7 (b) shows the enlarged view of the left edge of the simulated eye diagram of data symbols at the input of the slicer without and with the proposed jitter-based DFE. It is seen that the timing jitter of the data eye is within the allowed maximum jitter. The data conveyed to the channel by the transmitter is a pseudo-random data sequence.

Table 6.1 shows the performance of proposed maximum-jitter based adaptive DFE. 


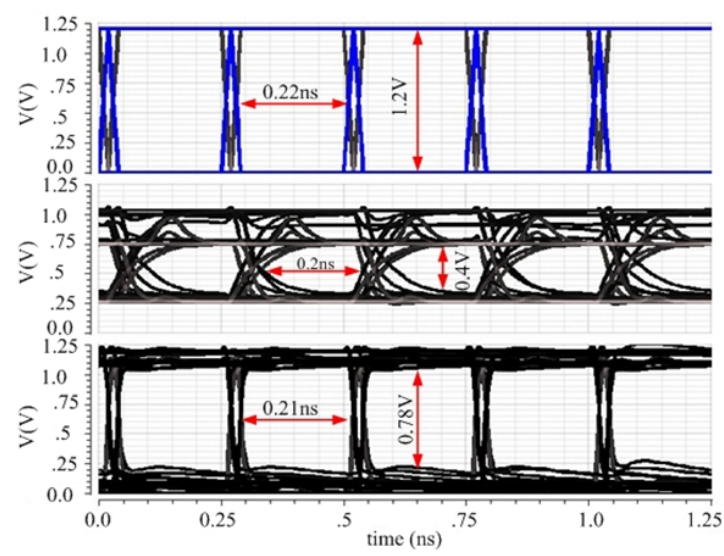

(a)

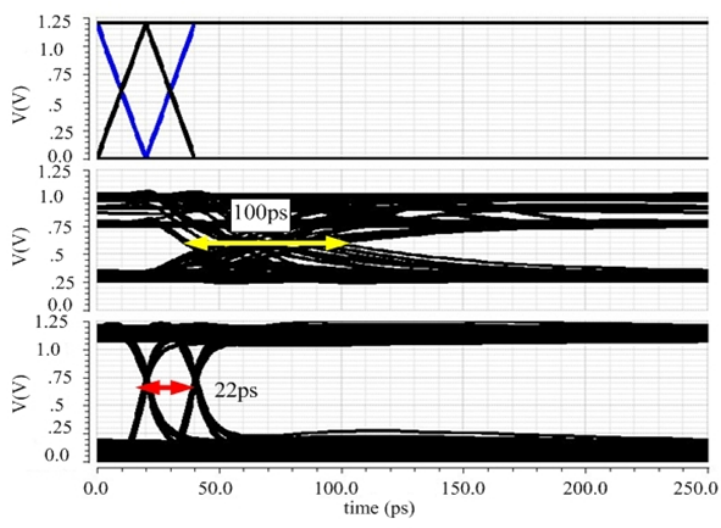

(b)

Figure 6.7: (a) : Simulated eye diagram of the data link. Channel length : $50 \mathrm{~mm}$. Top : Data conveyed to the channel. Middle: Data at the far-end of the channel without the proposed DFE. Bottom : Data at the far-end of the channel with the proposed DFE. (b) : The left edge of the simulated eye diagram of the data link. Channel length : $50 \mathrm{~mm}$. Top : Data conveyed to the channel. Middle : Data at the far-end of the channel without the proposed DFE. Bottom : Data at the far-end of the channel with the proposed DFE.

\subsection{Drawbacks of Adaptive DFE Using SS-LMS or EOM}

In this section, we investigate the drawbacks of adaptive DFE utilizing SS-LMS and EOM.

\subsubsection{Drawbacks of Adaptive DFE Using SS-LMS}

Although SS-LMS is widely used due to its simplicity, it suffers from some drawbacks. In general, SS-LMS provides error signals based on the difference between the equalized data symbol and the corresponding output signals of the slicer. The tap coefficients will be 
increased or decreased based on the sign of the error. Due to channel imperfections, the received signal is an analog signal and the equalization basically tries to return it to its digital form by comparing it with the output of the slicer. Since it is impossible to make both signals identical, the error is always non-zero. This means that SS-LMS will always provide either negative or positive error signals as shown in Fig.6.8 even if the adaptation process has already reached convergence time and the received data eye is sufficiently large, such that the data can be recovered safely resulting in unwanted power consumption.

A small step-size could be used in search for optimal tap coefficients to reduce power consumption when adaptation convergence. This, however, will lead to a long adaptation convergence time, as shown in Fig.6.9. Hence, a trade-off between the step-size and adaptation time must be made. This considers as another drawback of SS-LMS adaptive DFE.

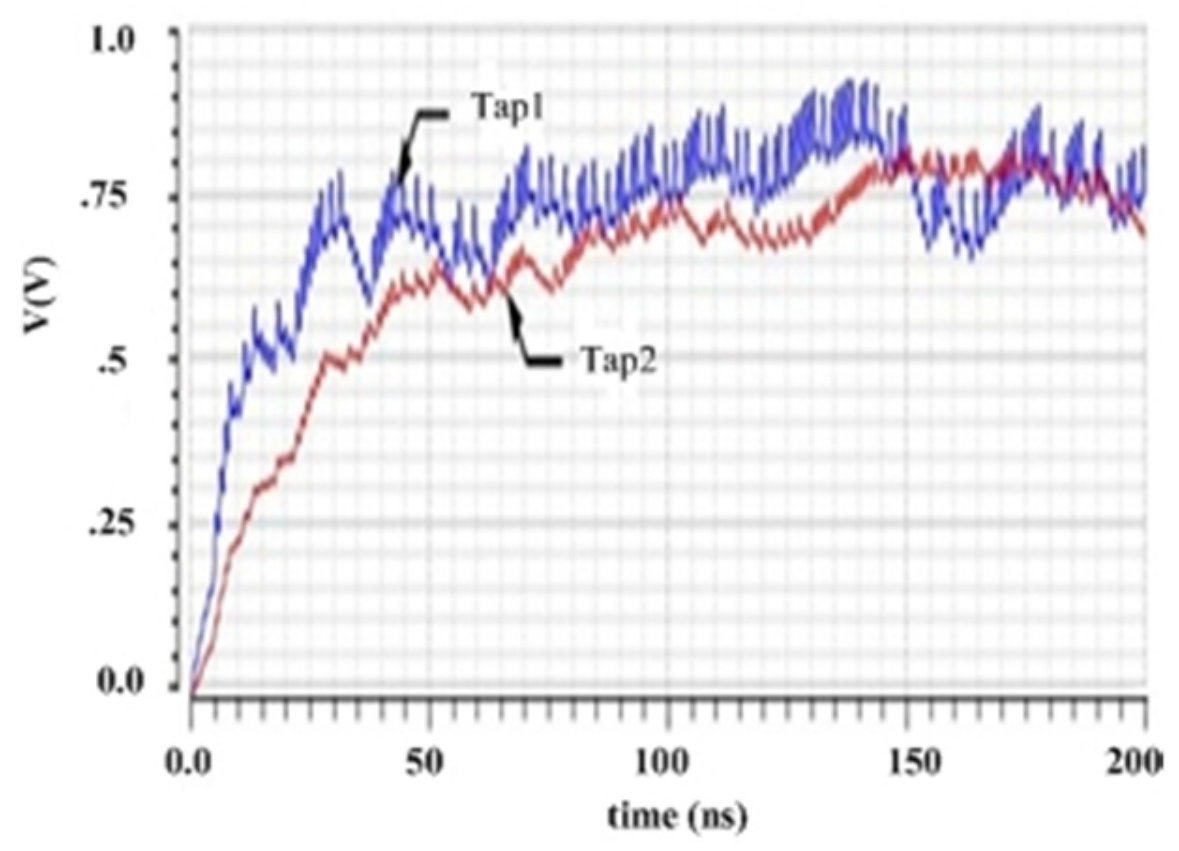

Figure 6.8: LMS tap coefficients. 


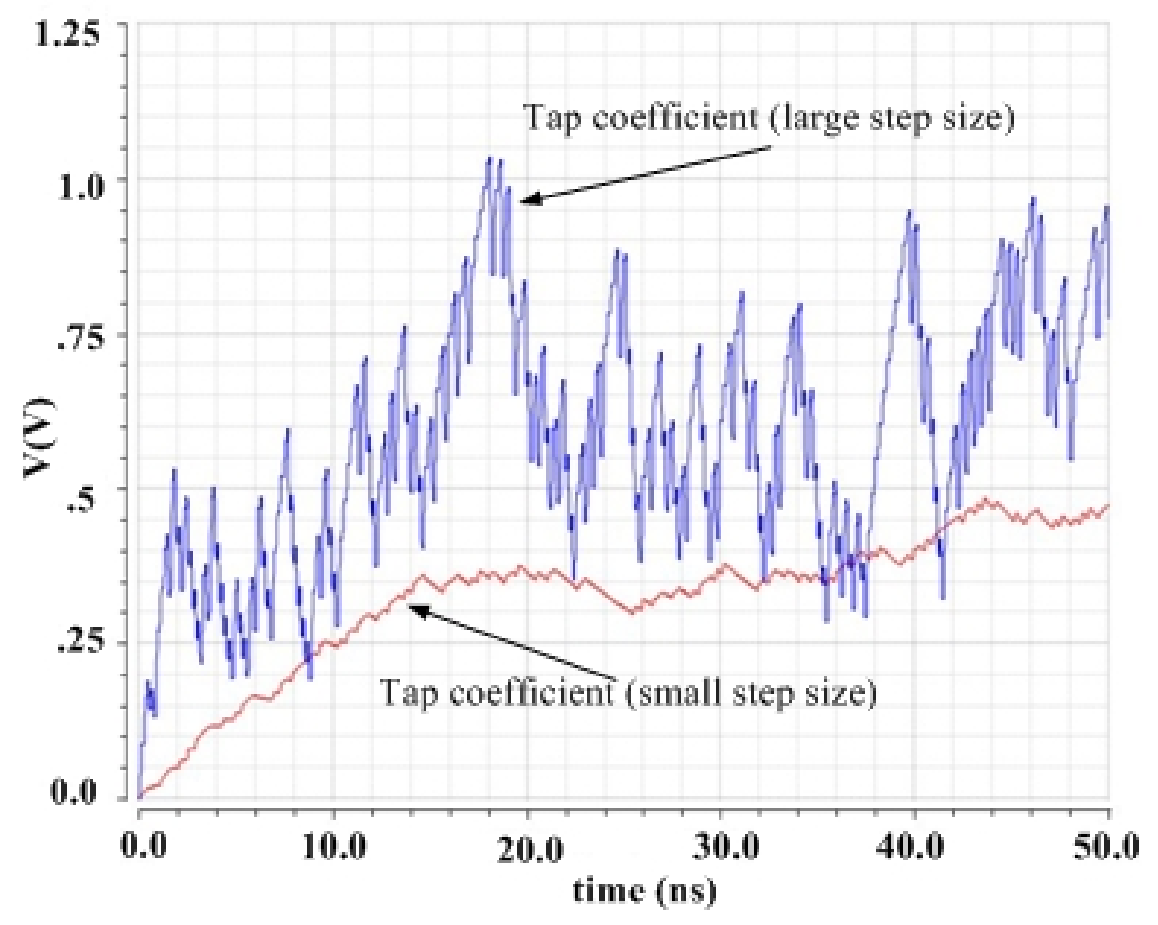

Figure 6.9: LMS step size.

\subsubsection{Drawbacks of Adaptive DFE Using EOM}

Eye-opening monitor extracts the error by sampling the received data symbols and compares the results with two threshold voltages $V_{H}$ and $V_{L}$. The violation with these threshold voltages at specific sampling time considers as an error. Since it only conveys the information on whether a violation of the minimum eye opening occurs or not, adaptation process moves only in one direction, as shown in Fig. 6.10. However, if a violation of the minimum eye opening occurs after the adaptation process completed, the adaptive engine will continue adjust the tap coefficients in the same direction. This leads to deteriorate the performance of the DFE as illustrated in Fig.6.11. 


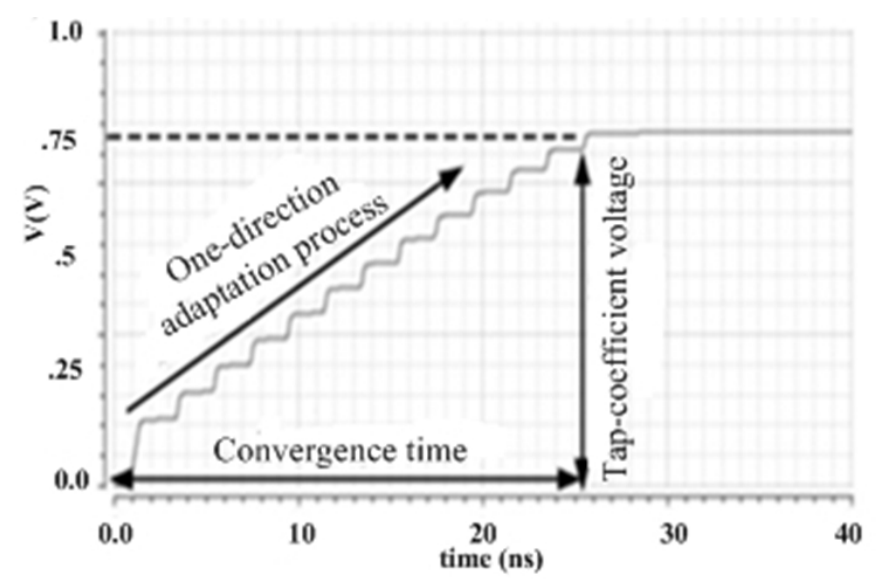

Figure 6.10: One-direction step size of EOM.

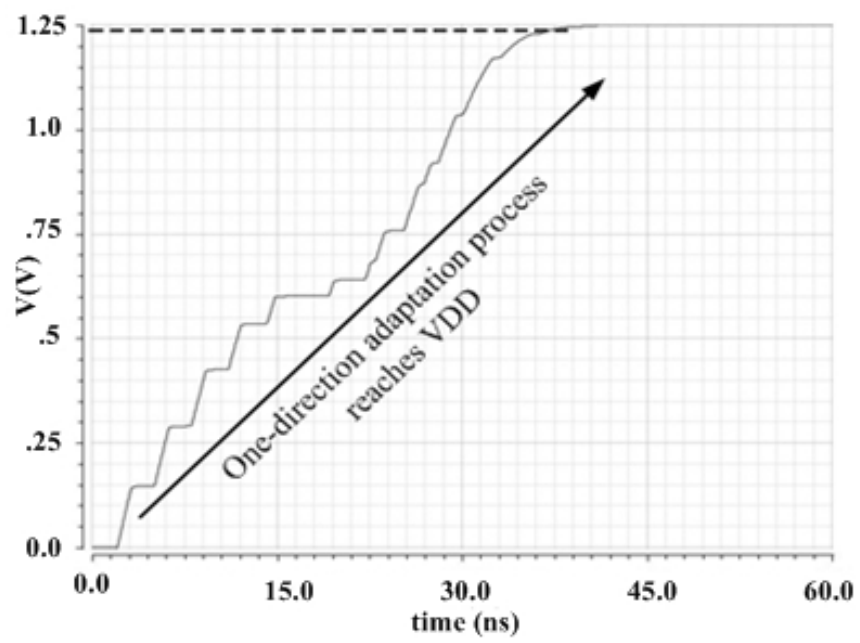

Figure 6.11: Drawback of one-direction step size of EOM.

\subsection{The Solution}

Digital-to-analog converter (DAC) gets its input signals from Error Detection Unit (EDU). The EDU generates error due to the violation of the received data symbol with minimum detection requirements at the clock and data recovery operation. Hence, an adaptive engine updates tap coefficients accordingly. In this section, we present a new adaptive DFE that eliminates the drawbacks of DFE using SS-LMS and EOM. As illustrated in Section 6.4.1 and Section 6.4.2 that DFE utilizing SS-LMS can adjust tap coefficients in both plus $(+)$ and minus (-) directions. It, however, continues to operate even the adaptation approaches 
the optimum. Whereas the DFE employing EOM determinates the operation when no error detected.

The schematic of proposed adaptive engine shown in Fig. 6.12 consists of two error detection units and one digital-to-analog converter. The schematic of EDUs is shown in Fig. 6.14.

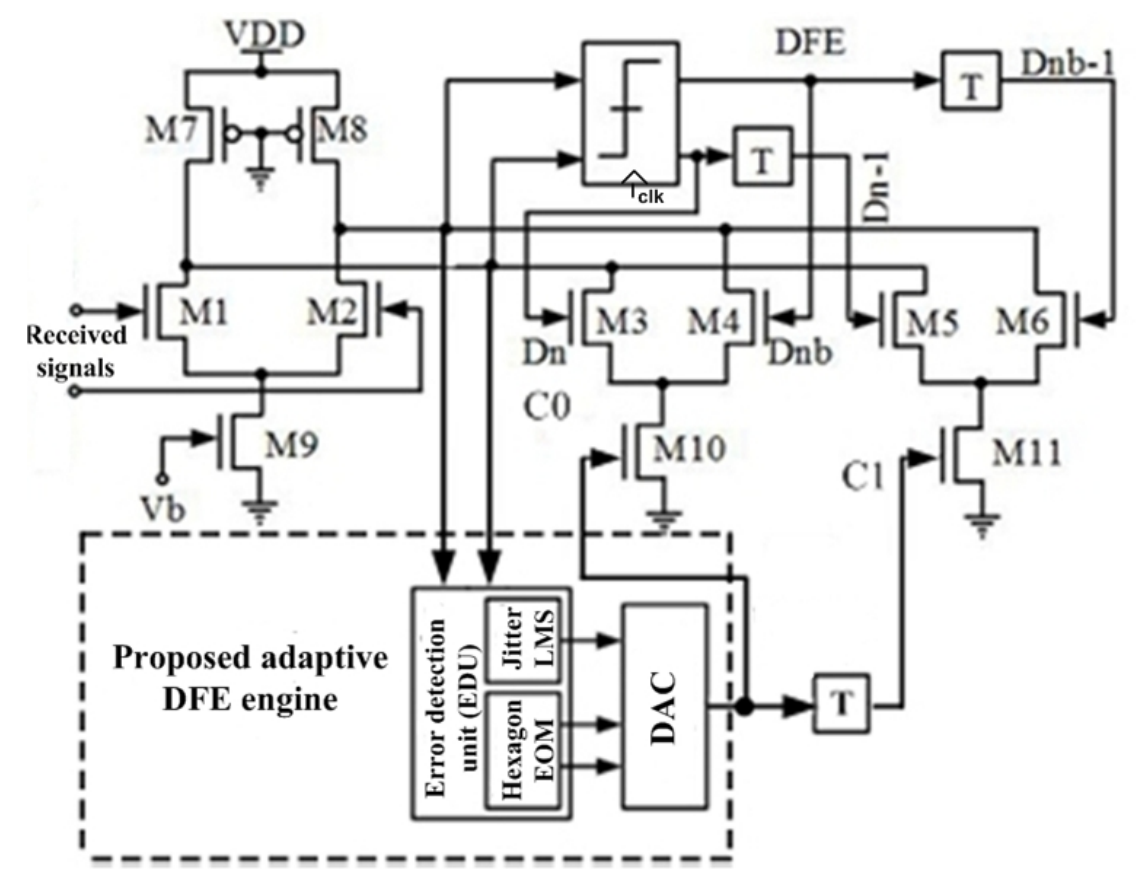

Figure 6.12: The schematic of proposed adaptive DFE including the proposed adaptive engine.

The first EDU detects the violation of the maximum timing jitter. It utilizes the least mean square algorithm to optimize the opening of the data eye. The second EDU utilizes a hexagon EOM to detect the error of the received data symbol, as detailed in Section 6.4.2. This EDU controls the ON/OFF of the adaptation process. Fig. 5.6 shows the DAC schematic which is considered in this adaptive engine. The input signal to the DAC is the error generated in two EDUs. A positive and negative step voltage are generated based on the error provided by the jitter EDU. The load capacitor of the DAC will be charged and discharged. This error signal enables the adaptive engine to provide positive and negative 
step voltages to update the tap coefficients of the DFE. The second EDU which utilizes half hexagon EOM controls the ON/OFF of the DAC. This EDU allows the adaptation process to update the tap coefficients as long as an error is detected by the EDU using EOM . When no error is detected, the opening of the received data symbol exceeds the minimum required a safe data recovery operation and the adaptive process ends. The flow chart of the search engine of he DFE is shown in Fig.6.13.

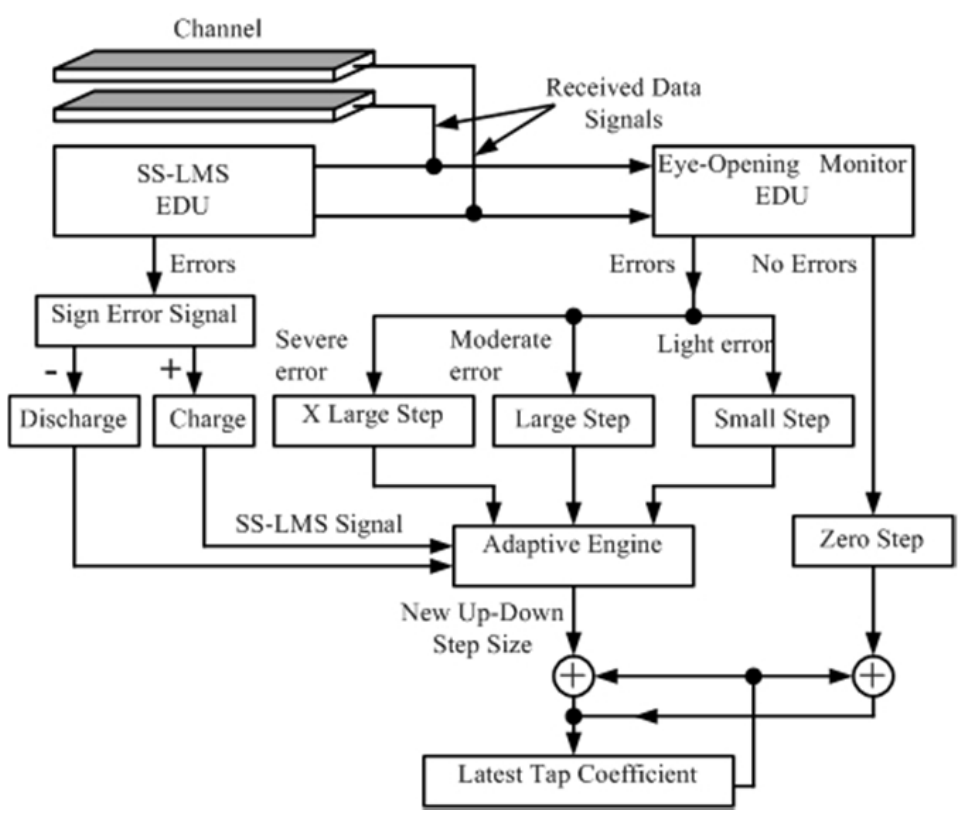

Figure 6.13: Search engine of proposed new adaptive engine for adaptive DFE.

\subsection{Simulation Results}

The effectiveness of the proposed adaptive engine AE of DFE is verified using a 1 Gbps serial link. The schematic of the combination of both Hexagon-based EDU and jitter-based EDU is illustrated in Fig.6.14. 


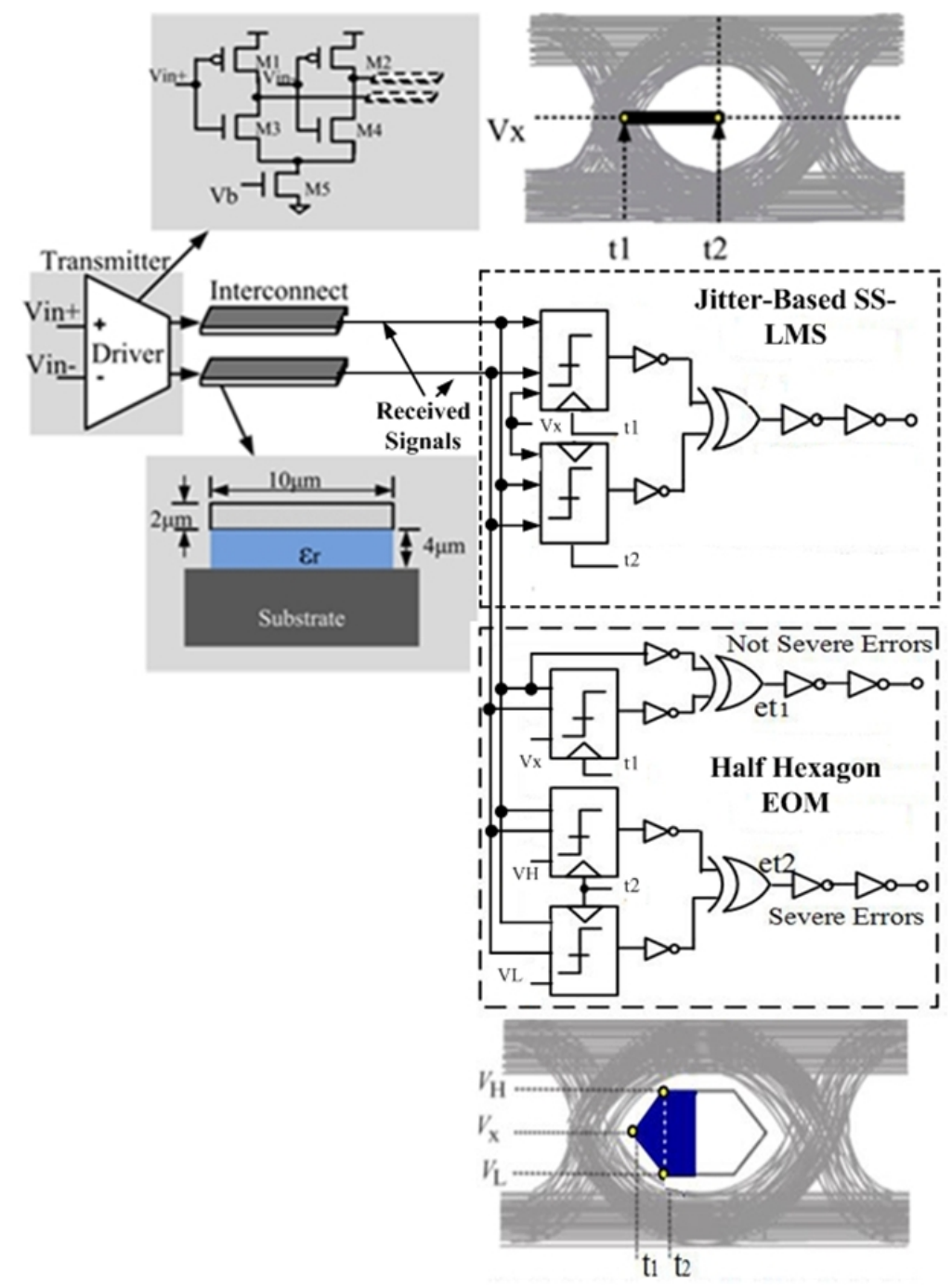

Figure 6.14: Serial link including two error detection units.

Differential current-mode driver is employed to convey a $2 \mathrm{~mA}$ current to the channel. The channel is a variable-length transmission line terminated with a $100 \Omega$ resistor. Jitter-based SS-LMS is illustrated at the top of the serial link schematic. Two slicers are used to compare the voltage of incoming data symbol with its common-mode voltage. The outputs of the slicers are XORed to generate the error signal. The error signal is fed to the input of DAC unit to provide a proper step voltage to adjust the tail currents of the feedback taps.

Three clocked comparators are used to implement the EDU utilizing a hexagon EOM 
shown at the bottom of the serial link schematic. Two error signals (severe and not sever) are extracted due to violation of received data signals and delivered to a DAC unit. $20 \mathrm{mV}$ is used as a one step level for adjusting the feedback taps. The severity, not severity, and both signals are $20 \mathrm{mV}, 40 \mathrm{mV}$, and $60 \mathrm{mV}$ respectively. $10 \mathrm{PF}$ capacitor is connected to the output to accumulate the voltage levels. Therefore, the tap coefficients will be adjusted for $20 \mathrm{mV}, 40 \mathrm{mV}$, and $60 \mathrm{mV}$ based on the error signal delivered from EDU using hexagon EOM and controls the $\mathrm{ON} / \mathrm{OFF}$ of the adaptation process as well. On the other hand, the error signal delivered from jitter-based EDU will make the tap coefficients to increase or decrease based on the sign of the error signal.

The simulation starts with increasing from zero to open the received data eye. different step sizes are drawn due to the severity and not severity signals. Also, we can see the up down of the signal due to the error signal delivered from EDU utilizing SS-LMS. This solved the drawback of using EOM for updating the tap coefficients in one direction. Also, there are accidental errors happened after the convergence time, this will not leads the adaptation process to hit the VDD. We can see after 45ns, the adaptation process is totally stopped due to the error signal provided from EDU using EOM. This eliminates the drawback of unlimitted error signal provided by using SS-LMS.

Figs.5.16-5.22 illustrate the schematic (left) and layout (right) of inverter, NOR2, XOR2, delay cell, clocked comparator, non-clocked comparator, adaptive engine, and DFE core respectively. Fig.6.23 represents the chip layout of the adaptive DFE utilizing maximum jitter based adaptive DFE technique. The chip layout $(1 \mathrm{~mm} \times 1 \mathrm{~mm})$ has been fabricated in Canadian Microelectronics Corporation (CMC) based on IBM 130nm 1.2V CMOS technology. 
Table 6.1: Performance of proposed jitter adaptive DFE (1 meter channel length).

\begin{tabular}{ll}
\hline Tech. & IBM $130 \mathrm{~nm}$ \\
Supply voltage & $1.2 \mathrm{~V}$ \\
Data rate & $2 \mathrm{Gbps}$ \\
Power of charge pumps & $3.41 \mathrm{~mW}$ \\
Power of error detect units & $6.33 \mathrm{~mW}$ \\
Power of DFE core (pre-amp., tap generators, delay units, ...) & $5.71 \mathrm{~mW}$ \\
Total power & $15.45 \mathrm{~mW}$ \\
Vertical eye-opening & $1.0 \mathrm{~V}(83 \%)$ \\
Horizontal eye-opening & $0.34 \mathrm{~ns}(68 \%)$ \\
Jitter & $80 \mathrm{ps}(16 \%)$ \\
\hline
\end{tabular}

\subsection{Chapter Summary}

A maximum jitter adaptive decision feedback equalizer was proposed for Gbps serial links. The adaptation is set by the detection of the violation of the maximum allowable jitter of received data symbols. The effectiveness of the proposed adaptive DFE was evaluated by embedding it in a 1 Gbps serial link with variable channel lengths implemented in an IBM $130 \mathrm{~nm}$ 1.2V CMOS technology. Simulation results demonstrated that the proposed adaptive DFE is capable of maximizing the eye-opening of received data symbols while meeting jitter constraints. Although the example used to validate the proposed adaptive DFE in this paper only contains two DFE taps, the underlining principle is valid for DFE with an arbitrary number of taps. The method presented here only applies to DFE with a fixed number of taps and cannot change the number of DFE taps adaptively. If one want to have the number of taps also adaptive to the characteristics of channels, an algorithm that can pre-determine the number of taps based on the characteristics of the channel is needed. 

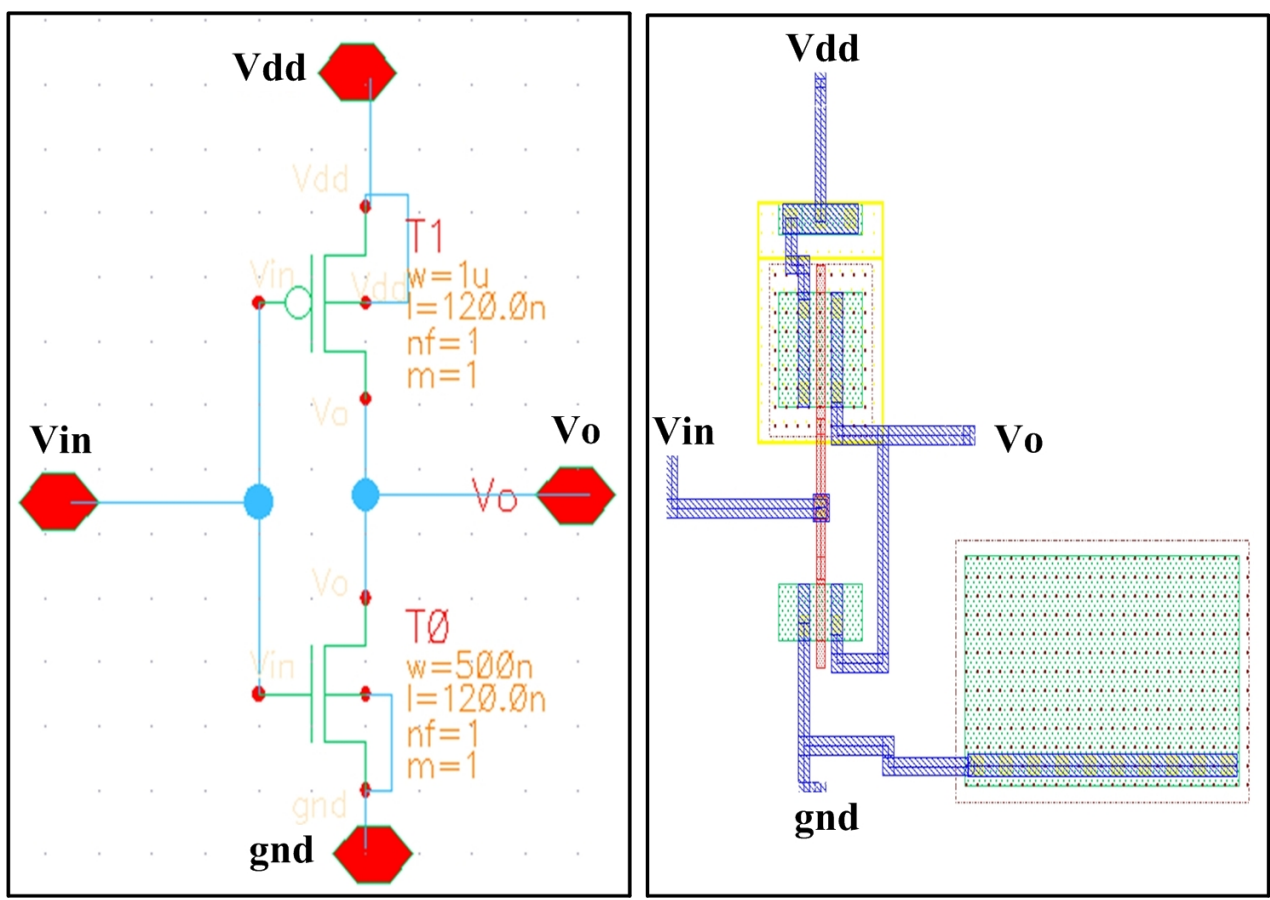

Figure 6.15: Inverter: schematic (left) and layout (right).
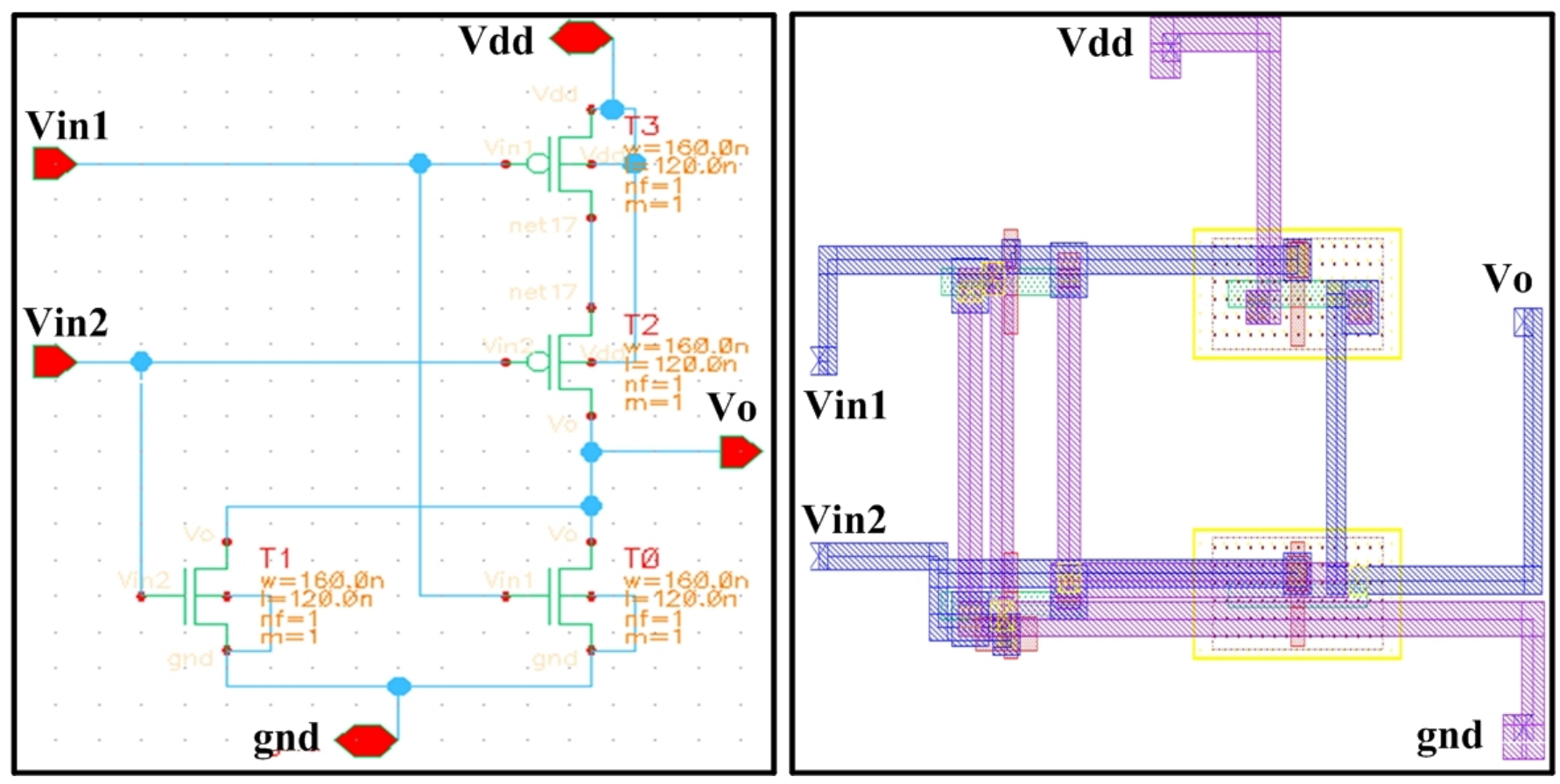

Figure 6.16: NOR2: schematic (left) and layout (right). 

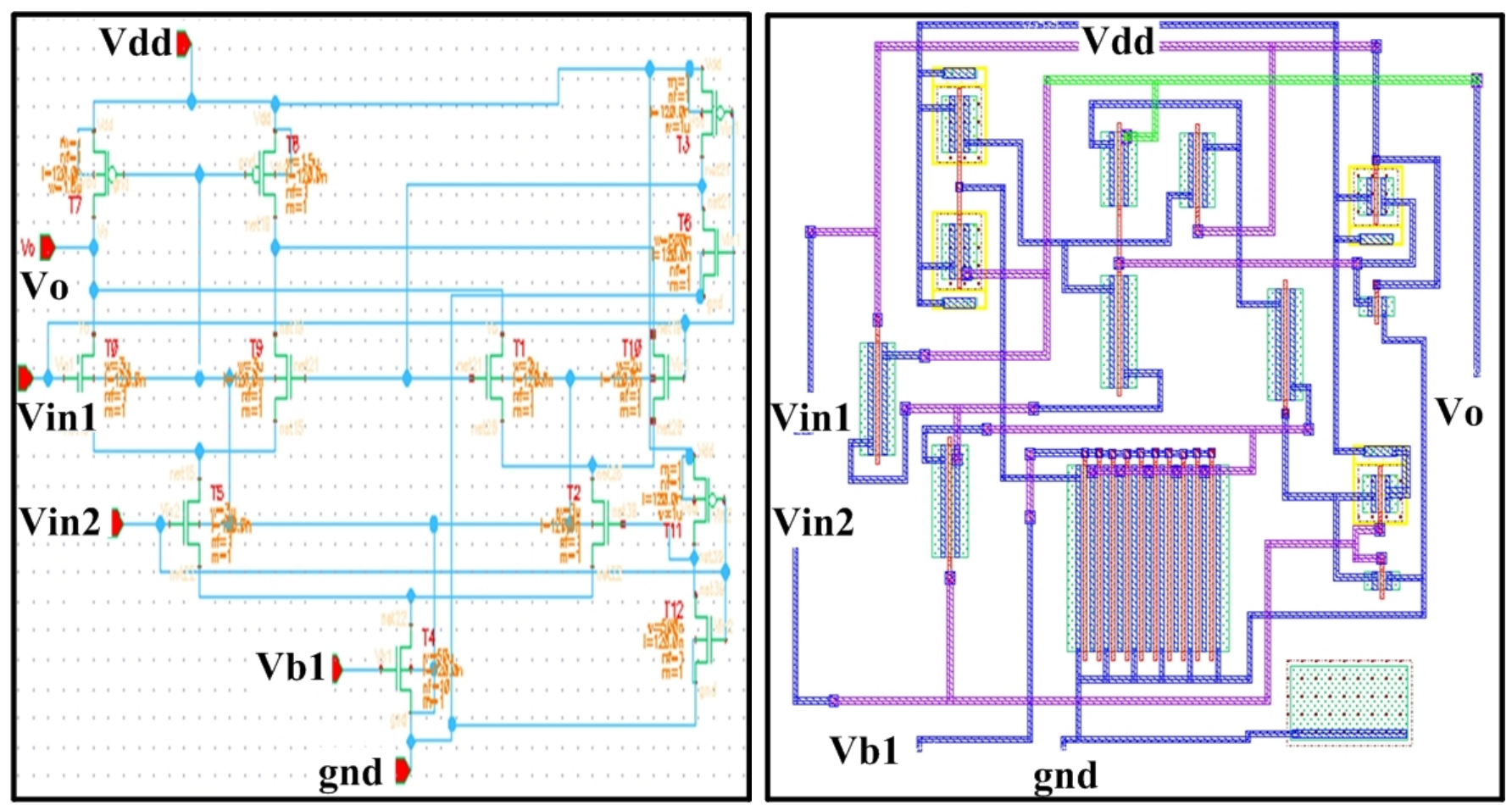

Figure 6.17: XOR2: schematic (left) and layout (right).
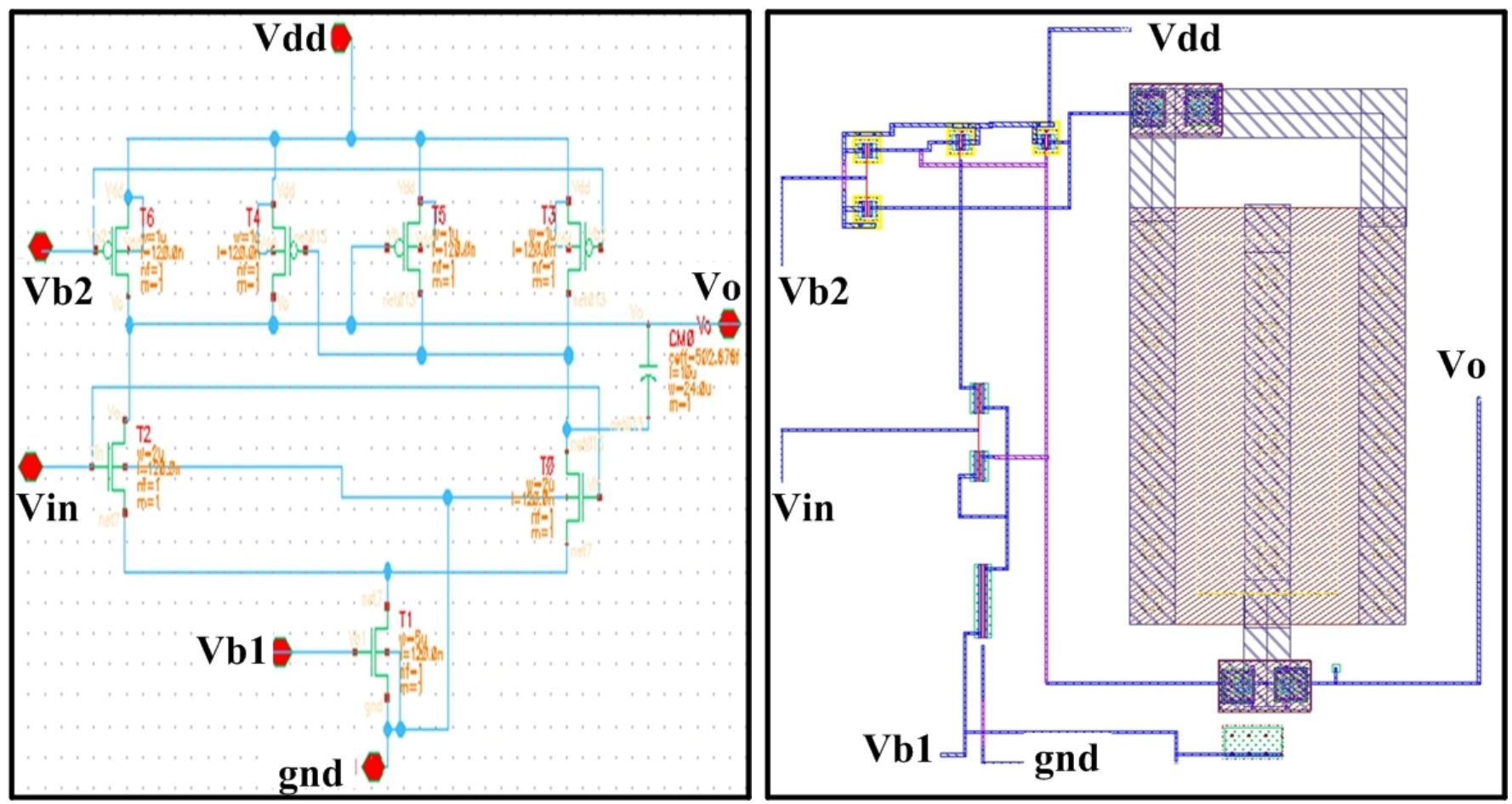

Figure 6.18: Delay cell: schematic (left) and layout (right). 

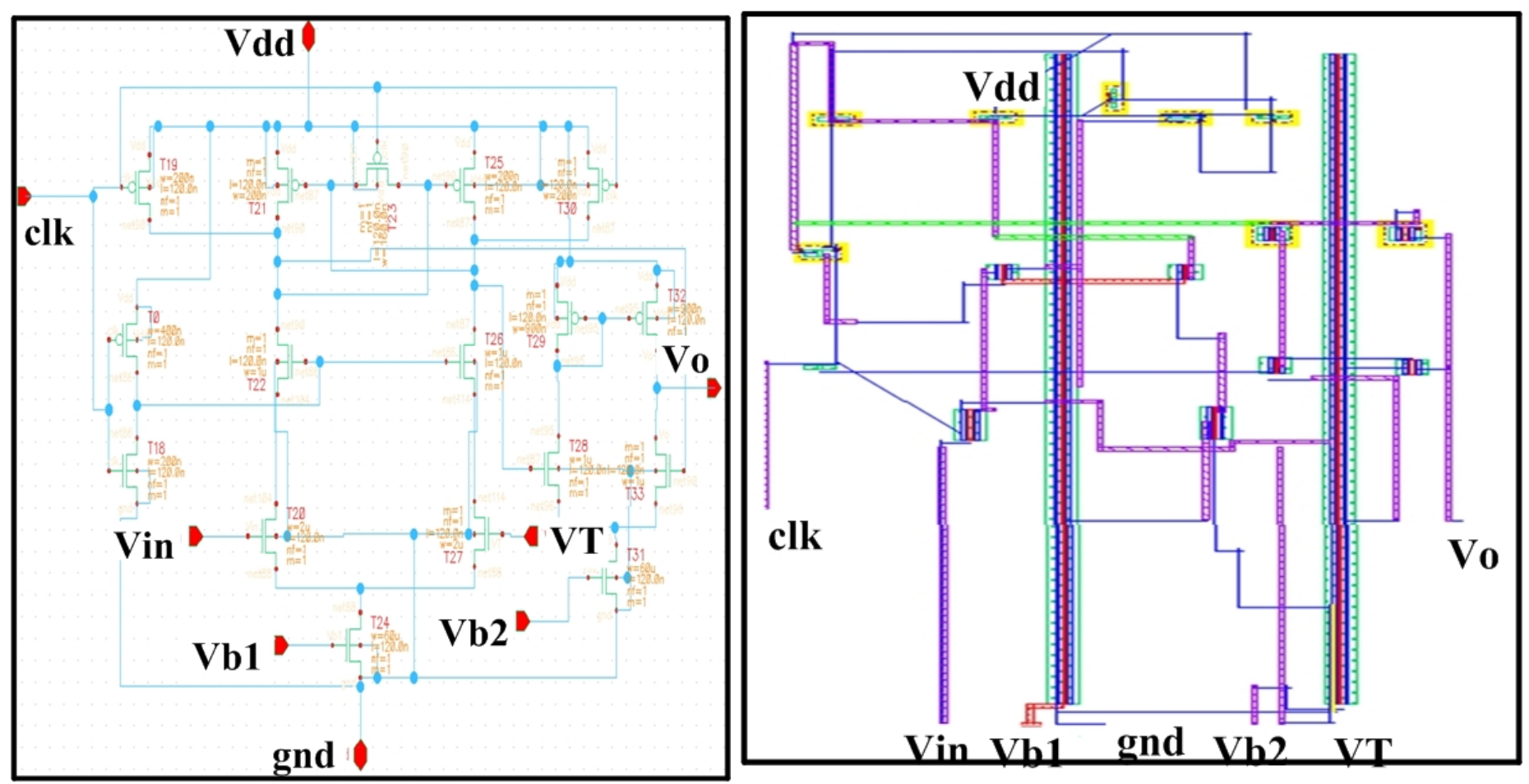

Figure 6.19: Clocked-comparator: schematic (left) and layout (right).
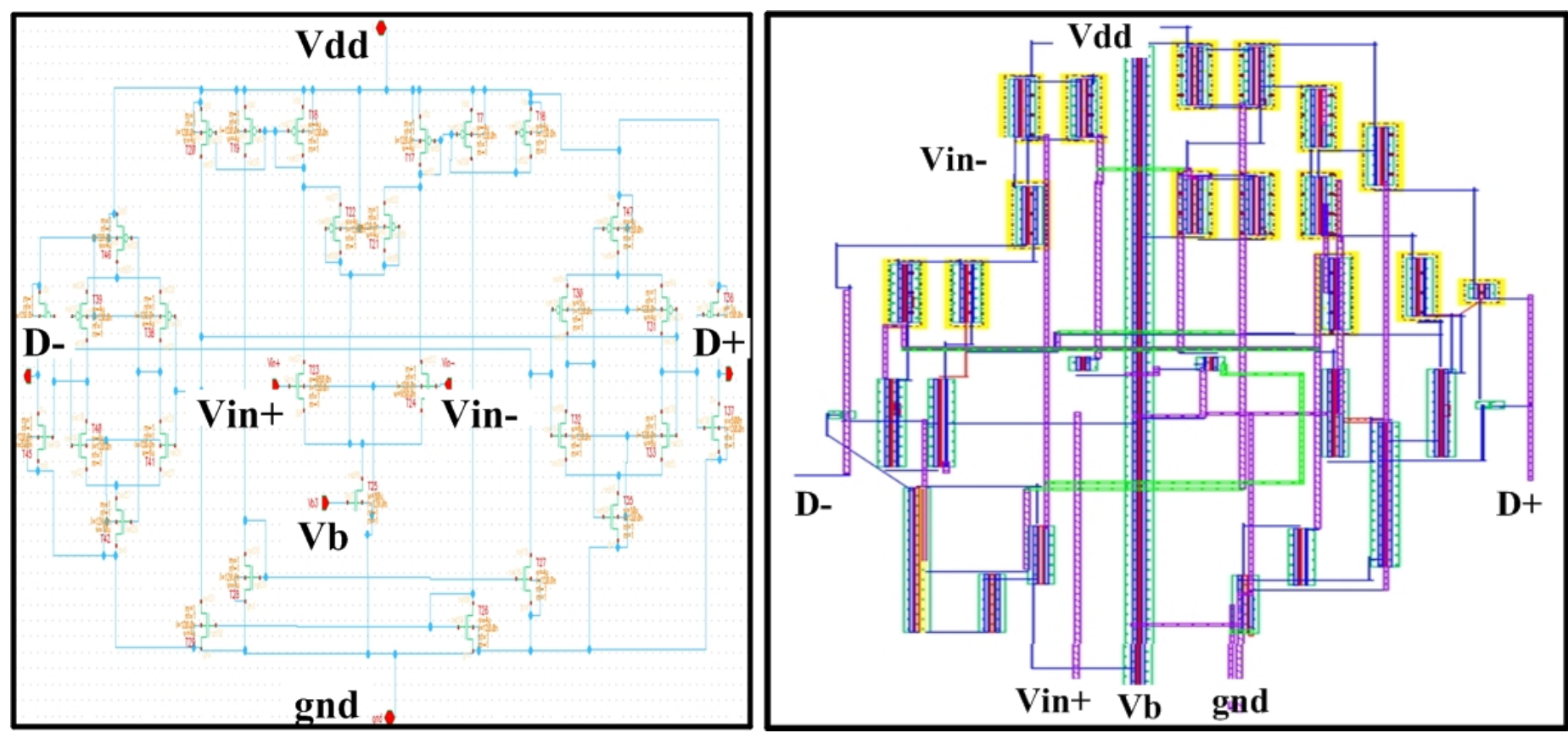

Figure 6.20: Non-clocked-comparator: schematic (left) and layout (right). 

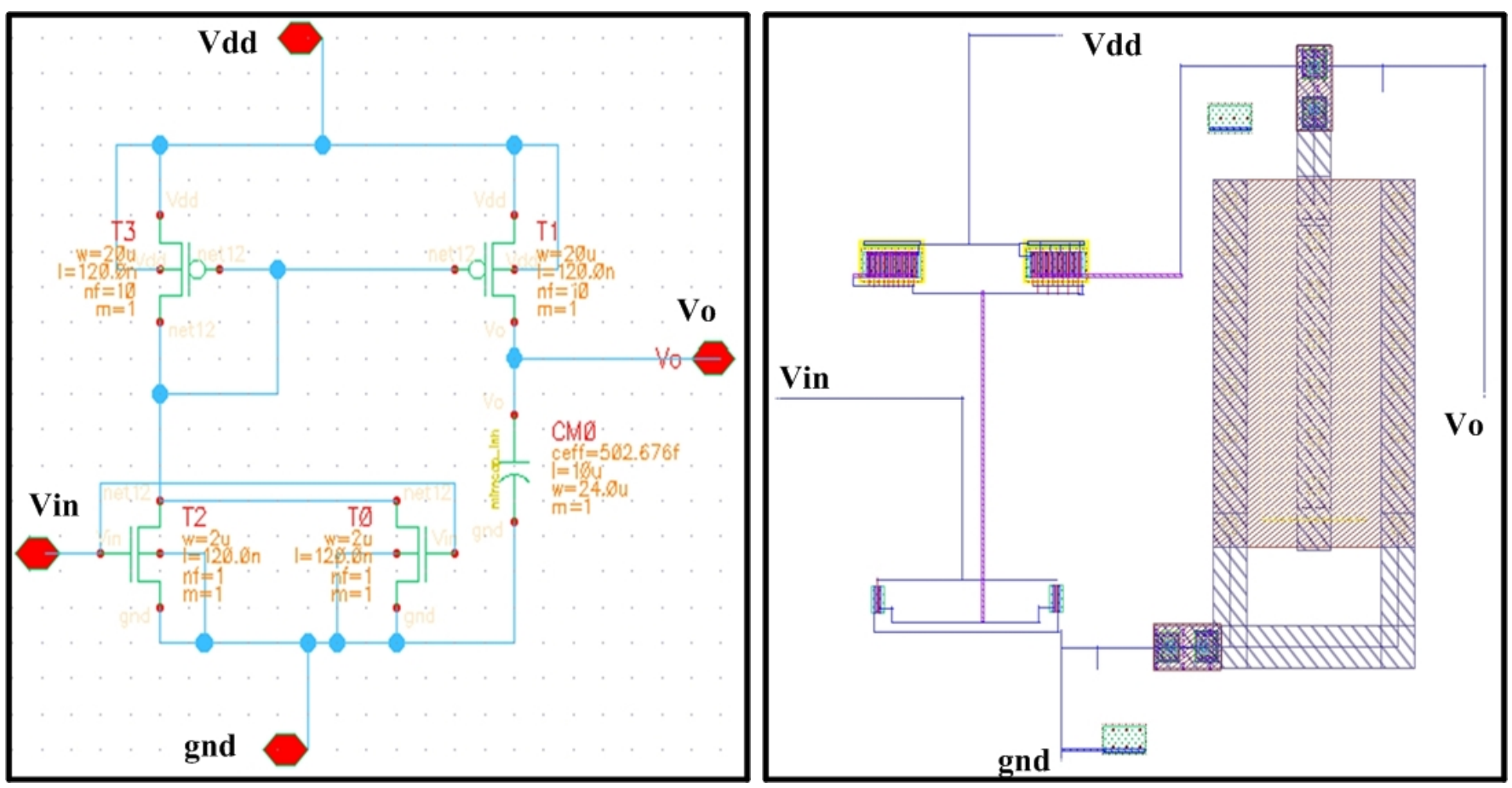

Figure 6.21: Adaptive engine: schematic (left) and layout (right).
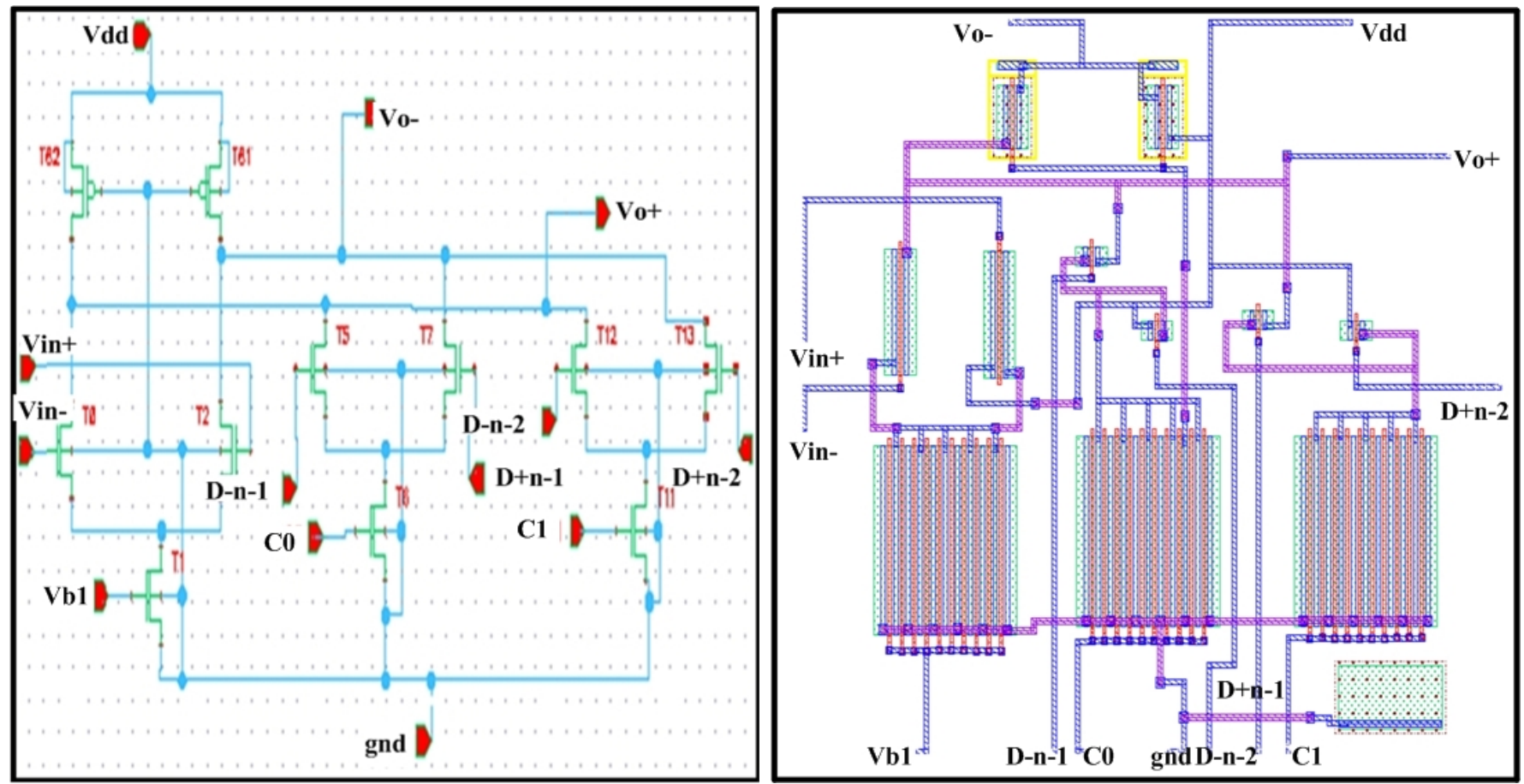

Figure 6.22: DFE core: schematic (left) and layout (right). 


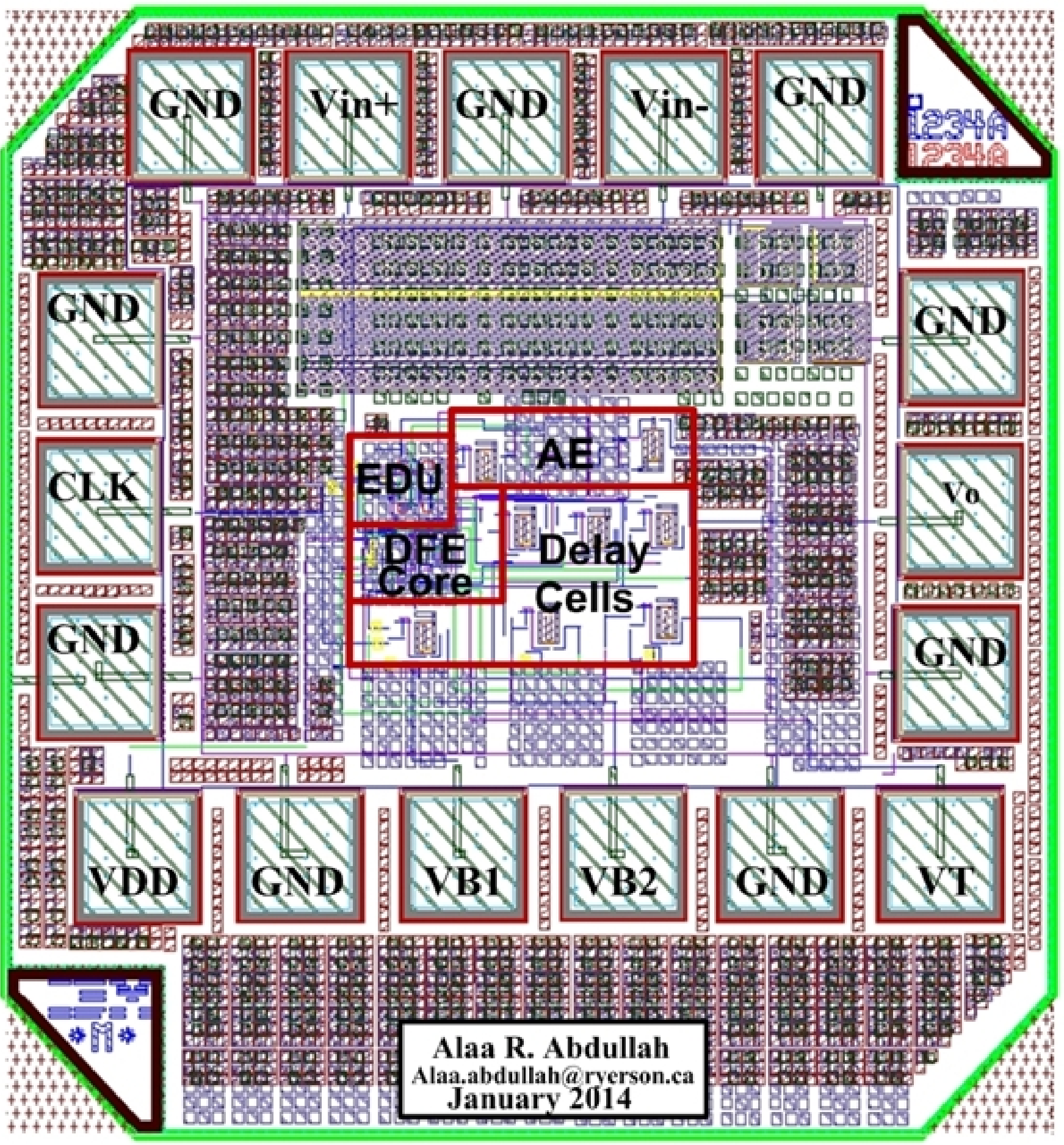

Figure 6.23: Layout of maximum-jitter adaptive DFE. 


\section{Chapter 7}

\section{Conclusions and Future Work}

\subsection{Conclusions}

In this dissertation, the imperfections of wire channels and their impact on data transmission were investigated. The fundamentals of near-end and far-end channel equalizations that combat the effect of the imperfections of wire channels were explored. A comprehensive review of decision feedback equalizers for Gbps data links was provided and a detailed examination of the principle, configuration, operation, and limitation of DFE was presented.

A hexagon two-dimensional eye-opening monitor (EOM) for quantifying the minimum eye-opening of received data was introduced. The proposed hexagon EOM outperforms rectangular counterparts by imposing a tighten constraint on data jitter at the edge of data eyes and eliminating unnecessary errors flagged by rectangular EOMs. Moreover, it is able to quantify the severity of the violation of the minimum eye-opening by received data so as to allow different step sizes to be used in search for optimal DFE tap coefficients, thereby improving performance and shortening DFE adaptation time.

To lower power consumption, a power-efficient half hexagon 2-dimensional EOM was introduced. The proposed half hexagon EOM outperforms the full hexagon EOM with comparable performance but greatly reduced silicon and power consumption.

An adaptive DFE utilizing the proposed hexagon EOM was developed. Similar to SSLMS adaptive DFE, the proposed DFE only needs Boolean signals, specifically, the severity of the violation of the minimum eye-opening and past decisions and is therefore computationally 
efficient. The different severities of the violation of the minimum eye-opening are mapped to different step sizes used in search for optimal DFE tap coefficients, thereby achieving better performance.

Finally, a power-efficient maximum jitter adaptive DFE was proposed. The adaptation in search for the optimal tap coefficients of DFE was set by the detection of the violation of the maximum jitter of received data symbols so as to satisfy the given maximum jitter constraints. The effectiveness of the proposed adaptive DFE was validated using simulation results.

\subsection{Future Work}

The methods presented here were only applied to DFE with a fixed number of taps and cannot change the number of DFE taps adaptively. If one wants to have the number of taps also changed adaptively, an algorithm that can pre-determine the number of taps based on the characteristics of the channel is needed. One possible solution to this problem is to monitor the slope of the post-cursor portion of data symbols as the number of the post-cursors is directly related to the slope of the impulse response of the channel in the post-cursor section.

Multi-level signaling utilized in serial links has been demonstrated to be an effective method for improving data rate. In this dissertation, the proposed hexagon and timingjitter EOMs were only validated in adaptive DFE for 2-PAM (pulse amplitude modulation) signal. They could be employed to monitor the validity of received multi-level signals as well due to the similarity of the effect of channel imperfections on both types of signals. This could be another research direction to improve the data rate of serial links. 


\subsection{List of Publications}

\section{Journal Papers}

1. Alaa R. AL-Taee, F. Yuan, and A. Ye, "A power-Efficient Maximum Jitter Adaptive Decision Feedback Equalizer for Gbps Serial Links," IET Circuits Devices Systems, revised in Feb. 2014.

2. Alaa R. AL-Taee, F. Yuan, and A. Ye, "Hexagon EOM-Based Variable Step-Size Adaptive DFE for Multi-Gbps Data Links," IEEE Transactions on Very Large Scale Integration Systems, revised in Jan. 2014.

3. F. Yuan, Alaa R. AL-Taee, and A. Ye, and S. Sadr, "Design techniques for decision feedback equalization of multi-Gbps data links: a state-of-the-art review, "IET Proceedings : Circuits, Devices, and Systems, Vol.8, No.2, pp. 118-130, 2014.

4. Alaa R. AL-Taee, F. Yuan, and A. Ye, "An improved RC model for VLSI interconnects with applications to buffer insertion," Analog Integrated Circuits and Signal Processing (Springer), Vol.79, No.1, pp. 105-113, April 2014.

5. Alaa R. AL-Taee, F. Yuan, A. Ye, and S. Sadr, "A New 2D eye-opening monitor for Gbps serial links," IEEE Transactions on Very Large Scale Integration Systems, Vol.22, No.6, pp. 1209-1218, June 2014.

6. Alaa R. AL-Taee, F. Yuan, A. Ye, "A Power-Efficient 2-Dimensional On-Chip EyeOpening Monitor for Gbps Serial Links," Analog Integrated Circuits and Signal Processing (Springer), Vol.76, No.1, pp.117-128, July 2013.

7. Alaa R. AL-Taee, F. Yuan, A. Ye, "A new power-efficient CDMA-based transmitter for high-speed serial links," Analog Integrated Circuits and Signal Processing (Springer), Vol.71, No.2, pp.1-7, Feb. 2012. 


\section{Conference Papers}

1. Alaa R. AL-Taee, F. Yuan, A. Ye, "Dual-mode adaptive decision feedback equalizer for multi-Gbps serial links," accepted for publication in IEEE RFIT, Hefei, China in July 2014.

2. Yushi Zhou, Alaa R. AL-Taee, F. Yuan, "Power-efficient dual-adaptive decision feedback equalizer for 4 PAM serial links," accepted for publication in IEEE RFIT, Hefei, China in July 2014.

3. Alaa R. AL-Taee, F. Yuan, A. Ye, "A New Adaptive Decision Feedback Equalizer Using Hexagon Eye-Opening Monitor for Multi-Gbps Data Links," IEEE Int'l Symp. on Circuits and Systems, Melbourne, Australia, Accepted for publication in Jan. 2014.

4. Alaa R. AL-Taee, F. Yuan, A. Ye, "Two-dimensional eye-opening monitor for serial links," Proc. IEEE MWSCAS, Columbus, pp.181-184, 2013.

5. Alaa R. AL-Taee, F. Yuan, A. Ye, "A New Simple RC Modeling for On-Chip Interconnects With its Applications to Buffer Insertion," Proc. SIECPC-2013, pp.1-4, 2013.

6. Alaa R. AL-Taee, F. Yuan, A. Ye, "A New CDMA Transmitter for High-Speed Serial Links," Peoc. SIECPC-2013, pp.1-4, 2013.

7. Alaa R. Abdullah, Adnan Kabbani and Kaamran Raahemifar, "AMapping The AWERLC Model Into a Simple RC Circuit With its Application to Buffer Insertion," IEEE Canadian Conference on Electrical and Computer Engineering (CCECE11), Niagara Falls, pp. 152-155, April, 2011. 


\section{Bibliography}

[1] A. Hu and F. Yuan, "signal timing skew compensation of parallel links with voltagemode incremental signaling," Trans. Circuits and Systems I., Vol. 56, no. 4, pp. 773-783, April 2009.

[2] P. Aziz, H. Kimura, A. Malipatil, and S. Kotagiri, " class of down-sampled floating tap dfe architectures with application to serial links," Proc. IEEE Intl Symp. Circuits and Systems, pp. 325-328, 2012.

[3] F. Zhong, S. Quan, W. Liu, P. Aziz, T. Jing, J. Dong, C. Desai, H. Gao, M. Garcia, G. Hom, T. Huynh, H. Kimura, R. Kothari, L. Li, C. Liu, S. Lowrie, K. Ling, A. Malipatil, R. Narayan, T. Prokop, C. Palusa, A. Rajashekara, A. Sinha, C. Zhong, and E. Zhang,

"1.0625-to-14.025 Gb/s multimedia transceiver with full-rate source-series-terminated transmit driver and floating-tap decision-feedback equalizer in $40 \mathrm{~nm}$ CMOS," IEEE Journal of Solid-State Circuits, Vol. 46, no. 12, pp. 3126-3139, 2011.

[4] F. Zhong, S. Quan, W. Liu, P. Aziz, J. Tai, J. Dong, C. Desai, H. Gao, M. Garcia, G. Hom, T. Huynh, H. Kimura, R. Kothari, L. Li, C. Liu, S. Lowrie, K. Ling, A. Malipatil, R. Narayan, T. Prokop, C. Palusa, A. Rajashekara, A. Sinha, C. Zhong, and E. Zhang, " $1.062514 .025 \mathrm{~Gb} / \mathrm{s}$ multi-media transceiver with full-rate source-series-terminated transmit driver and floating-tap decision-feedback equalizer in $40 \mathrm{~nm}$ CMOS," IEEE J. Solid-State Circuits, Vol. 46, no. 12, pp. 3126-3139, 2011.

[5] T. Dickson, J. Bulzacchelli, and D. Friedman, " $12 \mathrm{~Gb} / \mathrm{s} 11 \mathrm{~mW}$ half-rate sampled 5- 
tap decision feedback equalizer with current-integrating summers in 45-nm SOI CMOS technology," IEEE J. Solid-State Circuits, Vol. 44, no. 4, pp. 1298-1230, 2009.

[6] B. Song and C. David, " NRZ Timing Recovery Technique for Band-Limited Channels ," IEEE J. Solid-State Circuits, Vol. 32, no. 4, pp. 143-147, 1997.

[7] Z. Zhao, J. Wang, S. Li, and J. Chen, "A 2.5 Gb/s 0.13um CMOS current mode logic transceiver with pre-emphasis and equalization," International Conference on Digital Object Identifier, pp. 368-371, 2007.

[8] S. Kao and S. Liu, "A 20-Gb/s Transmitter With Adaptive Pre-emphasis in 65nm CMOS Technology," IEEE Trans. on Circuits and Systems, Vol. 57, no. 5, pp. 319-323, 2010.

[9] A. Fiedler, R. Mactaggart, J. Welch, and S. Krishnan, " 1.0625 Gb/s Transceiver with 2x-Oversampling and Transmit Signal Pre-Emphasis," IEEE Int. Solid-State Circuits Conference (ISSCC), pp. 238-239, 1997.

[10] W. J. Dally and J. Poulton, "Transmitter Equalization for 4-Gb/s Signaling," IEEE Micro, Vol. 17, no. 1, pp. 90-94, 1997.

[11] T.Beukema, M. Sorna, K. Selander, S. Zier, B. L. Ji, P. Murfet, J. Mason, W. Rhee, H. Ainspan, B. Parker, and M. Beakes, "A 6.4-Gb/s CMOS SerDes core with feed-forward and decision-feedback equalization," IEEE J. Solid-State Circuits, Vol. 40, no. 12, pp. 2633-2645, 2005.

[12] F. Yuan, "An area-power efficient 4-PAM full-clock 10 Gb/s CMOS pre-emphasis serial link transmitter," Analog Integrated Circuits and Signal Processing, Vol. 59, no. 3, pp. 257-264, 2009.

[13] K. Wong, E. Chen, and C. Yang, "Edge and data adaptive equalization of serial-link transceivers," IEEE J. Solid-State Circuits, Vol. 43, no. 9, pp. 2157-2169, 2008. 
[14] R. Mellitz, B. Samaras, and P. Williams, "Performing Adaptive External Equalization," US Pstent Application no. 20090110043, 2009.

[15] V. Stojanovic and et al., "Autonomous dual-mode (oam2/4) serial link transceiver with adaptive equalization and data recovery ,"IEEE J. Solid-State Circuits, Vol. 40, no. 4, pp. 1012-1026, 2005.

[16] B. Razavi, Design of integrated circuits for optical communications, New York: McGrawHill, 2003.

[17] K. Kammeyer, "Time truncation of channel impulse responses by linear filtering : A method to reduce the complexity of Viterbi equalization ,"Int. J. of Electronics and Communications (AEU), Vol. 48, no. 5, pp. 237-243, 1994.

[18] Y. Hidaka, W. Gai, T. Horie, J. Jiang, Y. Koyanagi, and H. Osone, "A 4-channel 1.25$10.3 \mathrm{~Gb} / \mathrm{s}$ backplane transceiver macro with $35 \mathrm{~dB}$ equalizer and sign-based zero-forcing adaptive control," IEEE J. Solid-State Circuits, Vol. 44, no. 12, pp. 3547-3559, 2009.

[19] M. Pozzoni and et al., "A multi-standard 1.5 to $10 \mathrm{~Gb} / \mathrm{s}$ latch-based 3-tap DFE receiver with a SSC tolerant CDR for serial backplane communication ,"IEEE J. Solid-State Circuits, Vol. 44, no. 4, pp. 1306-1315, 2009.

[20] V. Balan et al., "A 4.8 Gb/s Serial Link for Backplane Applications Using Decision Feedback Equalization," IEEE J. of Solid-State Circuits, Vol. 40, nO. 9, 2005.

[21] S. Gondi and B. Razavi, "Equalization and clock and data recovery techniques for 10 Gb/s CMOS serial-link receivers," IEEE J. of Solid-State Circuits, Vol. 42, no. 9, pp. 1999-2011, 2007.

[22] D. Lee, J. Han, G. Han, and S. Park, "10 Gbit/s 0.0065 mm2 6 mW analogue adaptive equalizer utilizing negative capacitance," IET Electronics Letters, Vol. 45, no. 17, pp. 863-865, 2009. 
[23] M. Austin, "Decision-feedback equalization for digital Communication Over Dispersive Channels," Research Laboratory of Electronics Technical Report 461, 1967.

[24] J. Zerbe and et al., "Equalization and Clock Recovery for 2 2.5-10Gbps 2-PAM/4PAM Backplane Transceiver Cell," IEEE J. of Solid-State Circuits, Vol. 38, no. 2, pp. 2121$2130,2004$.

[25] B. Kim, Y. Liu, T. Dickson, J. Bulzacchelli, and D. Friedman, "A 10-Gb/s compact lowpower serial I/O with DFE-IIR equalization in 65-nm CMOS," IEEE J. of Solid-State Circuits, Vol. 44, no. 12, pp. 3526-3538, 2009.

[26] W. Chen and G. Huang, "A parallel multi-pattern PRBS generator and BER tester for 40 Gbps SerDes applications," IEEE Proc. Conf. on Advanced System Integrated Circuis, pp. 318-321, 2009.

[27] T. Dickson, J. Bulzacchelli, and D. Friedman, "A 12 Gb/s $11 \mathrm{~mW}$ half-rate sampled 5tap decision feedback equalizer with current integrating summers in $45 \mathrm{~nm}$ SOI CMOS technology," IEEE J. of Solid-State Circuits, Vol. 44, no. 4, pp. 1298-1305, 2008.

[28] T. Toifl, C. Menolfi, M. Ruegg, R. Reutemann, D. Dreps, T. Beukema, A. Prati, D. Gardellini, M. Kossel, P. Buchmann, M. Brandli, P. Francese, and T. Morf, "A 2.6 mw/Gb/s 12.5 Gbps RX with 8-tap switched-capacitor DFE in $32 \mathrm{~nm}$ CMOS," IEEE J. Solid-State Circuits, Vl. 47, no. 4, pp. 897-910, 2012.

[29] K. Krishna and et al., "A 10-Gb/s 5-tap DFE/4-tap FFE trnsceiver in $90 \mathrm{~nm}$ CMOS technology," IEEE J. Solid-State Circuits, Vol. 41, no. 12, pp. 2885-2900, 2006.

[30] K. Krishna and et al., "A multi-giga-bit backplane transceiver core in 0.13-um CMOS with a power-efficient equalization architecture," IEEE J. Solid-State Circuits, Vol. 40, no. 12 , pp. 2658-2666, 2005.

[31] K. Wong, A. Rylyakov, and C. Yang, "A $5 \mathrm{~mW} 6$ Gb/s quarter-rate sampling receiver 
with a 2-tap DFE using soft decisions," IEEE J. Solid-State Circuits, Vol. 42, no. 4, pp. 881-888, 2007.

[32] S. Kasturia and et al., "Adaptive nonlinear cancellation for high-speed fiber- optic systems," Journal of Lightwave Technology, Vol. 10, no. 7, pp. 971-977, 1992.

[33] J. Ren and et al., "Precursor ISI reduction in high-speed I/O," IEEE Symp. on VLSI Circuits,pp. 134-135, 2007.

[34] R. Payne and et al., "A 6.25Gb/s binary adaptive DFE with first post-cursor tap cancellation for serial backplane communications," IEEE Int. Solid-State Circuits Conference, pp. 68-69, 2005.

[35] C. Yang, R. Farjad-Rad, and M. Horowitz, "A 0.5um CMOS 4.0-Gbit/s serial link transceiver with data recovery using oversampling," IEEE J. Solid-State Circuits, Vol. 33, no. 5, pp. 713-722, 1998.

[36] S. Sidiropoulos and M. Horowitz, "A $700 \mathrm{Mb} / \mathrm{s} /$ pin CMOS signaling interface using current integrating receivers," IEEE J. Solid-State Circuits, Vol. 32, no. 5, pp. 681-690, 1997.

[37] F. Yuan, current-mode circuits for data communications. New York: Springer, 2007.

[38] Y.Hidaka, W. Gai, T. Horie, J. Jiang, Y. Koyanagi, and H. Osone, "A 4- channel 1.25$10.3 \mathrm{~Gb} / \mathrm{s}$ backplane transceiver macro with $35 \mathrm{db}$ equalizer and sign-based zero-forcing adaptive control," IEEE J. Solid-State Circuits, Vol. 44, no. 12, pp. 3547-3559, 2009.

[39] B. Razavi, Deign of Analog CMOS Integrated Circuits. New York: McGraw- Hill, 2001.

[40] X. Zhou and et al, "A dynamic offset control technique for comparator design in scaled CMOS technology," Proc. IEEE Custom Integrated Circuits Conf., pp. 495-498, 2008.

[41] M. Lee, W. Dally, and P. Chiang, "Low-power area-efficient high-speed I/O circuit techniques," IEEE J. Solid-State Circuits, Vol. 35, no. 11, pp. 1591-1599, 2000. 
[42] Y. Ying and S. Liu, "A 20Gb/s digitally adaptive equalizer/DFE with blind sampling," IEEE Int. Solid-State Circuits Conference, pp. 444-446, 2011.

[43] S. McLeod, A. Sheikholeslami, T. Yamamoto, N. Nedovic, H. Tamura, and W. Walker, "A digital offset-compensation scheme for an la and cdr in 65-nm cmos," Symp. on VLSI Circuits, pp. 16-17, 2009.

[44] S. Ibranhim and B. Razavi, "Low-power CMOS equalizer design for 20-Gb/s systems," IEEE J. Solid-State Circuits, Vol. 46, no. 6, pp. 1321-1336, 2011.

[45] M. Park, J. Bulzacchelli, M. Beakes, and D. Friedman, "A 7 Gb/s 9.3 mW 2-tap currentintegrating DFE receiver," IEEE J. Solid-State Circuits, Vol. 22, no. 3, pp. 230-231, 2007.

[46] P. Payandelhnia, A. Abbasfar, S. Sheikhaei, B. Forouzandeh, K. Nanbakhsh, and A. Eghbali, "A $4 \mathrm{~mW}$ 3-tap $10 \mathrm{~Gb} / \mathrm{s}$ decision feedback equalizer," Proc. IEEE Mid-West Symp. on Circuits and Systems, pp. 1-4, 2011.

[47] A. Rontogiannis and K. Berberidis, "Efficient decision feedback equalization for sparse wireless channels," IEEE Trans. on Wireless Communications, Vol. 2, no. 3, pp. 570$581,2003$.

[48] J. Winters and R. Gitlin, "Electrical signal processing techniques in long-haul fiberoptic systems," IEEE Trans. Wireless Communications, Vol. 38, no. 9, pp. 1439-1453, 1990.

[49] T. Ellermeyer, U. Langman, B. Wedding, and W. Pohlmann, "A 10 Gb/s eye- opening monitor IC for decision-guided adaptation of the frequency response of an optical receiver," IEEE J. Solid-State Circuits, Vol. 35, no. 12, pp. 1958-1963, 2000.

[50] T. Suttorp and U. Langmann, "A 10-Gb/s CMOS serial-link receiver using eye-opening monitoring for adaptive equalization and for clock and data recovery," IEEE Custom Integrated Circuits Conf., pp. 277-280, 2007. 
[51] B. Analui, A. Rylyakov, S. Rylov, M. Meghelli, and A. Hajimiri, "A 10-Gb/s twodimensional eye-opening monitor in 0.13-um standard CMOS," IEEE J. Solid-State Circuits, Vol. 40, no. 12, pp. 2689-2699, 2005.

[52] M. Shin and et al., "A 6.4 Gbps on-chip eye opening monitor circuit for signal integrity analysis of high speed channel," IEEE Int. Symp. on Electromagnetic Compatibility, pp. $1-6,2008$.

[53] D. Hong and K. Cheng, "An accurate jitter estimation technique for efficient high speed I/O testing," Asian Test Symp., pp. 224-229, 2007.

[54] F. Gerfers, G. Besten, P. Petkov, J. Conder, and A. Koellmann, "A 0.2-2 Gb/s 6x OSR receiver using a digitally self-adaptive equalizer," IEEE J. Solid-State Circuits, Vol. 43, no. 6 , pp. 1436-1448, 2008.

[55] K. Wong, E. Chen, and C. Yang, "Edge and data adaptive equalization of serial-link transceivers," IEEE J. Solid-State Circuits, vol. 43, no. 9, pp. 2157-2169, Sept. 2008.

[56] B. Abiri, A. Sheikholeslami, H. Tamura, and M. Kibune, "A 5Gb/s adaptive DFE for 2x blind ADC-based CDR in 65 nm CMOS," IEEE J. Solid-State Circuit, Vol. 16, no. 9, pp. 436-437, 2011.

[57] B. Abiri, A. Sheikholeslami, H. Tamura, and M. Kibune, "An adaptation engine for a 2x blind ADC-based CDR in $65 \mathrm{~nm}$ CMOS," IEEE J. Solid-State Circuits, Vol. 46, no. 12, pp. $3140-3149,2011$.

[58] M. Harwood and et al., "A $12.5 \mathrm{~Gb} / \mathrm{s}$ SerDes in $65 \mathrm{~nm}$ CMOS using a baud-rate ADC with digital receiver equalization and clock recovery," . IEEE Int. on Custom Integrated Circuits Conf., pp. 436-437, 2007.

[59] A. AL-Taee, F. Yuan, A. Ye, and S. Sad, "A new two-dimensional eye-opening monitor for Gbps serial links," IEEE Trans. VLSI, no.99, pp.1, 2013. 
[60] Using the on-chip signal quality monitoring circuitry (EyeQ) feature in stratix IV transceivers. available at: http://www.altera.com/literature/an/an605.pdf: Altera Corp., 2012.

[61] F. Bien, H. Kim, Y. Hur, M. Maeng, J. Cha, S. Chandramouli, E. Gebara, and J. Laskar, "A $10 \mathrm{~Gb} / \mathrm{s}$ reconfigurable CMOS equalizer employing a transition detector-based output monitoring technique for band-limited serial links," IEEE Trans. on Microwave Theory and Techniques, Vol. 54, no. 12, pp. 4538-4547, 2006.

[62] H. Noguchi, N. Yoshida, H. Uchida, M. Ozaki, S. Kanemitsu, and S. Wada, "A 40Gb/s CDR circuit with adaptive decision-point control based on eye-opening monitor feedback," IEEE J. Solid-State Circuits, Vol. 43, no. 12, pp. 2929-2938, 2008.

[63] A. Fayed and M. Ismail, "A low-voltage low-power CMOS analog adaptive equalizer for UTP-5 cables," IEEE Trans. Circuits and Systems I., Vol. 55, no. 2, pp. 480-495, 2008.

[64] M. Marcu, S. Durbha, and S. Gupta, "Duty-cycle distortion and specifications for jitter test-signal generation," Proc. IEEE Int. Symp. on Electromagnetic Compatibility, pp. $1-4,2008$.

[65] T. Carusone, D. Johns, and K. Martin, Analog integrated circuit design. New York: John Wileys and Sons, 2008.

[66] Y.Hidaka, W. Gai, T. Horie, J. Jiang, Y. Koyanagi, and H. Osone, "A 4-channel 1.25$10.3 \mathrm{~Gb} / \mathrm{s}$ backplane transceiver macro with $35 \mathrm{~dB}$ equalizer and sign-based zero-forcing adaptive control," IEEE J. Solid-State Circuits, Vol. 44, no. 12, pp. 3547-3559, 2009.

[67] T. Carusone, D. Johns, and K. Martin, Analog integrated circuit design, 2nd ed. New York: John Wileys and Sons, 2012.

[68] S. Shahramian, H. Yasotharan, and A. Carusone, "Decision feedback equalizer architectures with multiple continuous-time infinite impulse response filters," IEEE Trans. Circuits and Systems II, Vol. 59, no. 6, pp. 226-230, 2012. 
[69] B. Leibowitz and et al., "A 7.5Gb/s 10-tap DFE receiver with first tap partial response, spectrally gated adaptation, and 2nd-order data-filtered CDR," IEEE J. Solid-State Circuits, Vol. 14, no. 9, pp. 228-599, 2007.

[70] C. Seong, J. Rhim, and W. Choi, "A 10-Gb/s adaptive look-ahead decision feedback equilizer with an eye-opening monitor," IEEE Trans. Circuits and Systems II, Vol. 59, no. 4, pp. 209-213, 2012.

[71] Y. Huang and S. Liu, "A 6 Gb/s receiver with 32.7dB adaptive DFE-IIR equalization," IEEE J. Solid-State Circuits, Vol. 11, no. 6, pp. 356-58, 2011.

[72] D. Turker, A. Rylyakov, D. Friedman, S. Gowda, and E. Sanchez-Sinencio, "A 19 Gb/s $30 \mathrm{~mW}$ 1-tap speculative DFE receiver in $90 \mathrm{~nm}$ CMOS," IEEE Symp. on VLSI Circuits and Systems, pp. 216-217, 2008.

[73] D. Turker, A. Rylyakov, D. Friedman, S. Gowda, and E. Sanchez-Sinencio, "A 78 mW $11.1 \mathrm{~Gb} / \mathrm{s} 5$-tap DFE receiver with digitally calibrated current-integrating summers in 65nm CMOS," IEEE J. Solid-State Circuits, Vol. 7, no. 6, pp. 368-369, 2009.

[74] S. Sarvari, T. Tahmoureszadeh, A. Sheikholeslami, H. Tamura, and M. Kibune, "A 5 $\mathrm{Gb} / \mathrm{s}$ speculative DFE for 2x blind ADC-based receivers in 65-nm CMOS," IEEE Symp. on VLSI Circuits and Systems, pp. 69-70, 2010.

[75] D. Turker, A. Rylyakov, D. Friedman, S. Gowda, and E. Sanchez-Sinencio, "A $78 \mathrm{~mW}$ $11.1 \mathrm{~Gb} / \mathrm{s} 5$-tap DFE receiver with digitally calibrated current-integrating summers in 65nm CMOS," IEEE J. Solid-State Circuits, Vol. 7, no. 6, pp. 368-369, 2009.

[76] H. Wang and J. Lee, "A 21-Gb/s 87-mW transceiver with FFE/DFE/analog equalizer in 65-nm CMOS technology," IEEE J. Solid-State Circuits, Vol. 45, no. 4, pp. 909-920, 2010.

[77] T. Dickson, Y. Liu, S. Rylov, B. Dang, C. Tsang, P. Andry, J. Bulzacchelli, H. Ainspan, X. Gu, L. Turlapati, M. Beakes, B. Parker, J. Knickerbocker, and D. Friedman, "An 8x 
10-Gb/s source-synchronous I/O system based on high-density silicon carrier interconnects," IEEE J. Solid-State Circuits, vol. 47, no. 4, pp. 884-896, April 2012.

[78] M. Nazari and A. Emami-Neyestanak, "A 15-Gb/s 0.5-mW/Gbps two-tap DFE receiver with far-end crosstalk cancellation," IEEE J. Solid-State Circuits, Vol. 47, no. 10, pp. 2420-432, 2012.

[79] G. Gangasani and et al., "A 16-Gb/s backplane transceiver with 12-tap current integrating DFE and dynamic adaption of voltage offset and timing drifts in 45-nm SOI CMOS technology," IEEE J. Solid-State Circuits, Vol. 47, no. 8, pp. 1828-1841, 2012.

[80] G. Gangasani and et al., "A 19-Gb/s serial link receiver with both 4-tap FFE and 5-tap DFE function in 45-nm SOI CMOS," IEEE J. Solid-State Circuits, Vol. 47, no. 12, p. 321-329, 2012.

[81] A. Amirkhany, K. Kaviani, A. Abbasfar, S. Fazeel, W. Beyene, C. Hoshino, C. Madden, K. Chang, and C. Yuan, "A 4.1-pJ/b, 16-Gb/s coded differential bidirectional parallel electrical link," IEEE J. Solid-State Circuits, Vol. 47, no. 12, pp. 3208-3219, 2012.

[82] F. Zhong and et al., "1.0625-to-14.025 Gb/s multimedia transceiver with full-rate sourceseries-terminated transmit driver and floating-tap decision-feedback equalizer in $40 \mathrm{~nm}$ CMOS," IEEE J. Solid-State Circuit, Vol. 22, no. 8, pp. 348-349, 2011.

[83] A. Joy and et al., " Analog-DFE-based 16 Gb/s SerDes in 40 nm CMOS that operates across $34 \mathrm{~dB}$ loss channels at Nyquist with a baud rate CDR and 1.2 Vpp voltage-mode driver," IEEE J. Solid-State Circuit, Vol. 22, no. 6, pp. 350-351, 2011.

[84] D. Cui and et al.," A dual-channel 23-Gbps CMOS transmitter/receiver for 40-Gbps RZ-DQPSK and CS-RZ-DQPSK optical transmission," IEEE J. Solid-State Circuits, Vol. 47, no. 12, pp. 3249-3260, 2012.

[85] F. Spagna and et al., " A $78 \mathrm{mw} 11.8 \mathrm{~Gb} / \mathrm{s}$ serial link transceiver with adaptive RX 
equalization and baud-rate CDR in $32 \mathrm{~nm}$ CMOS," IEEE J. Solid-State Circuits, Vol. 41, no. 3, pp. 366-367, 2010.

[86] T. Toifl, C. Menolfi, M. Ruegg, R. Reutemann, D. Dreps, T. Beukema, A. Prati, D. Gardellini, M. Kossel, P. Buchmann, M. Brandli, P. Francese, and T. Morf," A 2.6 mw/Gb/s 12.5 Gbps RX with 8-tap switched-capacitor DFE in $32 \mathrm{~nm}$ CMOS," IEEE J. Solid-State Circuits, Vol. 47, no. 4, pp. 897-910, 2012.

[87] J. Bulzacchelli and et al., " A 28-Gb/s 4-tap FFE/15-tap DFE serial link transceiver in 32-nm SOI CMOS technology," IEEE J. Solid-State Circuits, Vol. 47, no. 12, pp. 22-35, 2012.

[88] J. Savoj, K. Hsieh, P. Upadhyaya, F. An, A. Bekele, S. Chen, X. Jiang, K. Lai, C. Poon, A. Sewani, D. Turker, K. Venna, D. Wu, B. Xu, E. Alon, and K. Chang, “ A wide common-mode fully-adaptive multi-standard $12.5 \mathrm{~Gb} / \mathrm{s}$ backplane transceiver in $28 \mathrm{~nm}$ CMOS," IEEE Symp. on VLSI Circuits and Systems, pp. 104-105, 2012.

[89] K. Hyoungsoo, J. de Ginestous, F. Bien, K. Lee, S. Chandramouli, Y. Hur, C. Scholz, E. Gebara, and J. Laskar, "An electronic dispersion compensator (EDC) with an analog eye-opening monitor (EOM) for $1.25 \mathrm{~Gb} / \mathrm{s}$ gigabit passive optical network (GPON) upstream links," IEEE Trans. on Microwave Theory and Techniques, Vol. 55, no. 12, pp. 2942-2950, 2007.

[90] D. Bhatta, K. Kim, E. Gebara, and J. Laskar, " A 10 Gb/s two dimensional scanning eye opening monitor in $0.18^{-} \mathrm{m}$ CMOS process," IEEE Int. Symp. on Microwave Symp. Digest, pp. 1141-1144, 2009. 